\title{
Directory of Awardee Names
}

July 1999

Prepared by

Office of Procurement and Assistance Management Information Systems and Analysis Division

and

Office of Sclentiflc and Technical Information 
Avaikablo to DOE and DOE contractors from the Office ol Scientific and Techulcal Information, P.O. Box 62, Oak Ridge, TN 37831; proes avatilable from (4te3) 576-8401. 


\section{DISCLAIMER}

This report was prepared as an account of work sponsoted by an ageney of the United States Government. Neither the United States Government nor any agency thereof, nor any of their employees, make any warranty, express or implied, or assumes any legal liability or responsibility for the accuracy, completeness, or usefuiress of any information, apparatus, product, or process disclosed, or represents that its use would not infringe privately owned rights. Reference herein to any specific commercial product, process, or service by trade name, trademark, manufacturer, or otherwise does not necessarily constitute or imply its endorsement, recommendation, or fevoring by the United States Government or any agency thereaf. The views and opinions of authors expressed herein do not necessarily state or reflect those of the United States Government or any agency thereof. 


\section{DISCLAIMER}

Portions of this document may be illegible in electronic image products. Images are produced from the best available original document. 


\section{Directory of Awardee Names}

\section{ABOUT THIS PUBLICATION}

Standardization of granl and contract awardee names has been an area of concern since the development of the Department's Procuretnent and Assistance Data \$ystem (PADS). A joint effort was begun in 1983 by the Office of Scientific and Technical Information (OST) and the Office of Procurement and Assistance Management/Informalion Systems and Analysis Division to develop a means for providing uniformity of awardee names.

As a result of this effort, a method of assigning vender identification codes to each unique awardec nante, division, city, and state combination was developed and is maintained by OSTI. Changes to vencor identification codes or awardee names contained in PADS can be made only by OSTI.

A wardee names in the Directory indicate that the uwardec has had a prime contract (excluding purchase orders of $\$ 10,000$ or less) with, or a financial assistance award from, the Department. Award status-active, inactive, or retiredis nol shown. The Directory is in alphabetic sequence based on awardee name and reflects the OSTI-assigned vendor identification code to the right of the name. A vendor identification code is assigned to each unique awardec name, division, city, and state (for place of periormance). The same vender identification code is used for awards throughout the Department.

The vendor identification codes are an effective method of assuring consistercy of names in the PADS system. The numbers are assigned as follows: lhe first six characters are known as the stem code and jdentify the awardee; the seventh character is a dash; the eighth and ninth characters identify the division of the swardee. If there is no division, these characters will be 00 . The first division that receives a vendor identification code will be 01 , and numnbers will be assigned in segquence to each different division. The tenth and eleventh characters, which represent the city and state, are assigned in the same way, with the first city and state bejng assigned 01, etc. The futhal two characters are a dash and a compuler-generaled check digit.

Information on new awardees (lhose which do nol yet have vendor ideatification codes in PADS) is sent daily from PADS to OSTI for assignment of codes. OSTI compares the new awardee names to those already in the Dirtetory and uses the "Instruclions for Completing Awardee Names in PADS" (Appendix D to the Handbook for Preparation of the Individual Procurement Action Report of July 1991) to standardize names and assign either an existing or a new vendor identification code.

The Directory is intended as a resource to be consultied whenever an initial award is to be entered into PADS.

The Disectory will be revised every three (3) years, and supplements will be issued quarterly. Each supplement will contain all new awardee names and codes since the last Directory was issued so that there are only two books to be consulted at any one time. When a new supplement is issued, the previous one should be discarded. 


\section{DIRECTORY OF AWARDEE NAMES}

\author{
Identiflextion \\ Awardee \\ code
}

A

A T KEARNEY INC

Chicago. LL

$000140-0002-4$

ABB COMBUSTION ENGINEERING INC Windsor, CT

ABB POWER T \& D CO INC

265000-000]-3

Tulsa, OK

195070-0023-2

ABELITY ENGINEERING TECH INC

South Holland, IL

ABMP TECH CORP

Amherst, NY

AC INDUSTRIAL SERVICES

Chico, CA

ACACIA BIOSCIENCES INC

Richmond, CA

ACL SERVICES LTD

Vancouver, Canada

ACME CONSTRUCTION CO Cody, WY

ACR ENGINEERING INC

Anthony, TX

Fort Gordon, GA

Gulf Breezt, FL

Kertville, TX

Mobile, AL

ADDISON CONSTRUCTION CO

Essex, MO

Esles Park, CO

Jonesboro, AR

Nogales, $A Z$

Pocahontas, AR

Wintier, SD

ADDISON DR LESEIE M

Baton Rouge, LA

ADROIT SYSTEMS INC

Alexandria, VA

ADT SECURITY SERYLCES INC

Pittsbutgh, PA

ADT SECURITY SYSTEMS

Tulsa, OK

ADVANCE

Whittier, $C A$

$283990-0001-9$

289450-0001-1

292220-0001-3

290910-0001-5

292960-0001-7

$284600-0001-X$

246400-0003-0

246400-0007-3

246400-0006-5

246400-0005-7

246400-000-4-9

000670-0032-0

000670-0034-7

000670-0036-3

000670-0037-]

000670-0033-9

000670-0035-5

$265210-0001-2$

$288950-000 \mathrm{~J}-3$

292880-0001-1

223970-0002-4

ADVANCED COMMUNICATJONS \& ELEC

Albuquerque, NM

ADVANCED CONTROL SYSTEMS INC

Norcross, GA

Springfield, MO

ADVANCED ENERGY SYSTEMS INE

Wilton, $\mathrm{NH}$

ADVANCED ENVIRONMENTAL TECH

Sbcrwood, OR

$256530-0002-7$

ADVANCED FUEL RESEARCH INC

East Hartfond, CT

000780-0002-5
Awardee

Identification

code

ADVANCED GLASS INDUSTRIES INC

R \& D

Rochester, NY

$280290-0101-0$

ADVANCED INTEGRATED MGMT SERV

Arvada, $\mathrm{CO}$

259450-0002-7

Goker, CO

259450-0003-5

ADVANCED MAGNET LABORATORY INC

Palm Bay, FL

284440-000t-9

ADVANCED MECHANICAL TECH INC

Watertown, MA $\quad 000810-0002-2$

ADVANCED MODULAR POWER SYSTEMS

Ann Arbor, Ml

259000-0001-X

ADVANCED MODULAR SPACE INC

Loveland, $\mathrm{CO}$

$265100-0001-6$

ADVANCED STUDY INSTITUTE FOR

Granby, VT

ADVANCED TECH \& LABS INTL

Germantown, MD

269900-0001-5

ADVANCED TECH SYSTEMS

Pittsburgh, PA

$256070-0002-8$

ADVANCED TECHNOLOGIES MGMT INC

Cleveland, OH

$292180-0001-2$

ADVANCED TECHNOLOGY INSTTTUTE

North Charleston, SC

290220-0001-X

AECL TECHNOLOGIES INC

Gaithersburg, MD

237777)-0002-7

AEG ELECTRIC POWER SYSTEMS INC Chatlexoi, PA

AEROMET INC

Tulsa, OK

AEROSOL DYNAMICS INC

Berkeley, CA

AGCY FOR TOXIC SUBSTANCE \& DIS

Atlanta, GA

AGE ELECTRICAL POWER SYSTEMS

Charleroi, PA

AGRICULTURE DEPARTMENT OF

Huron, SD

$253930-0001-4$

$292720-0001-7$

Springfield, IL

Admin Dis

Boise, ID

Cibola National Forest

Tijeras, NM

Forest Service

Flagstaff, AZ

Fresno, CA

Mackay, ID

Pocatello, ID

Portland, OR

Redding, CA

Williams, AZ

Natural Resounces Conserv Ser

East Lansing, MI
2867201-0001-3

001090-00A3-5

$001090-0006-X$

001090.0107 .9

001090-3599-2

D01090-07A0-9

(001090-0716-6

001090-0795-6

001090-0794-8

001090-0730-1

001090-0788-3

$00] 050-0787-5$

001090-3198-9 
Awardete

Identification code

Natural Resources Canservation

Tomple, TX

Savannah River Natl Resource

New Ellenton, SC

Sol Prodnctivity in the South

Research Triangle Park, NC

AIR FORCE DEPARTMENT OF

Hanscona AFB, MA

Hanson, MA

Midwest City, OK

Ogden, UT

Robins Air Force Base, GA

15th Air Base Wing

Honolulu, HI

377th Civil Eng Group

Albuguerque, NM

547th Intedigence Squad

Nellis AFB. NV

9yth Air Base Wing

Nelilis AFB, NV

Air Warfare

Las Vegas, NV

Defense Speclal Weapons Schood

Kirtland AFB, NM

Edwards Air Force Base

Edwands, CA

Maxelt AFB

Gumiersville, AL

Resenrch Laloratory

Albuquerque, NM

Special Operations Conamand Hurlburt Field, FL

$001090.36 \mathrm{AL}-\mathrm{C}$

001090-37A2-E

$001090-3497 \cdot X$

$001230-0056-4$

001230-0051-3

$001230-0065-3$

001 230-0069-6

col 230-0067-X

$001230-8049-5$

$001230-7901-2$

$001230-7714-1$

$001230-7614-5$

$001230-6032-X$

$001230-7466-5$

$001230.7364-2$

$001230-7568-8$

$001230-8101-7$

$001230-7870-9$

AIR PRODUCTS \& CHEMICALS INC

Gos 혼 Equipment Group

Alkentown, PA

$001260-080 \mathrm{~L}-9$

ATRK ENGINEERING INC New Castle, VA

A.jS MOSAIC DISPLAY SYSTEM Warten, $M$

AKZO NOAEL INC

Dobbs Ferry, NY

ALABAMA AYIATION \& TECH COLL

Ozark, AL

$289150-0001-3$

$163960-0001-6$

291150-0001-X

(x)1400-0001-1

ALABAMA CRYOGENIC ENGINEERING Huntsville, AL

$099060-0001-5$

ALABAMA UNIVERSITY OF

Materials \& Mechanteal Engr

Bimingharn, AL.

Sponsoned Programs Office of

Tuscaloosa, AL

ALAMEDA CTR FOR ENVIRON TECH

Alameda, CA

$001450-2301-X$

ALAN M VOORHEES \& ASSOC MeLeas, VA

ALASKA HOUSING FINANCE CORP

Anchorage, AK

Rural Reteanch \& Development

Anchorage, AK
Awardee

Identification

code

ALASKA VILLAGE ELECTRIC CORP

Anchorage, AK

289700-0001-1

ALBAN ENGINE POWER SYSTEM

Elkridge, MD

ALBANY CITY OF

Water Department

Albany, OR

ALBANY STATE UNIVERSITY

Albany, GA

$287770-0001-3$

ALBER CORP

Boca Raton, FL

$001610-0202-1$

ALBERTSON J A

Nyssa, OR

$288500-0000]-4$

$291920-0001=0$

ALCATEL NETWORK SYSTEMS INC

Federal Engipeering

Richardson, TX

$225000-0101-6$

ALCHEMET INC

Aston, PA

$289160-0001-7$

ALCOA FUJIKURA LTD

Mill Valley, CA

Spartanburg, SC

ALCORN CO SCHOOL BD

Corinth, MS

$172540-0004-1$

$172540-0005-X$

$281030-0001-9$

ALEXANDER BARBARA A

Winthrop, ME

$286370-0001-0$

ALFRED UNIVERSITY

Center For Glass Reseirch

Alfred, NY

$001850-0301-\mathrm{X}$

ALIESAN JODY

Seatte, WA

$270720-0001-1$

ALIX RICHARD E

Rowe, MA

ALL STATE FAB \& WELDING

Stockton, CA

$277290-80011-6$

ALE-STAR

Silver Spring, MD

$287480-000 t-9$

ALLIED SIGNAL AEROSPACE CO

Torrance. CA

ALLIED-SIGNAL COMPOSTTES INC

Newark, DE

ALLED-SIGNAL INC

Torrance, CA

ALLMEDIA ELECTRONICS

Golden, $\mathrm{CO}$

$2915000001-2$

ALPHONS VENANCIO G

Nashyille, TN

Pontape / Caroline Islands,

ALTERNATTVE ENERGY GROUP INC

New Haven, CT

AM INSTITUTE FOR LEARNING

Austin, TX

AMARCH CORP

Houston, TX

$291510-0001-6$

165680-0003-5

$262090-0002-9$

$281470-0001-4$

$281470-0002-2$

279450-0001-0

$286540.0001-5$

AMER LUNG ASSOC OF METRO CHIC

Chicago, IL

$286160-0001-1$

AMERICAN BATTERY CHARGING INC

Smilhfield, RI

288050-0000]-9 
Awardee $\begin{gathered}\text { Identification } \\ \text { corle }\end{gathered}$

AMERICAN CHEMICAL SOCIETY

Salt Lake City, UT

002560-0008-5

AMERICAN INDIAN SCI \& ENG SOC

Boulder, $\mathrm{CO}$

$139660-0001-6$

AMERICAN INST OF CHEMICAL ENG

Chem Proc Safety Center for

New York, NY $256320 \sim 010)-6$

AMERICAN IRON \& STEEL INST

Pittsburgh, PA

184280-0002-6

AMERICAN JUDICATURE SOCIETY

Chicago, IL 291970-0001-9

AMERICAN MATERIAL HANDLING

Lilburn, GA 288460-0001-3

AMERICAN MOBHLE SATELLTTE CORP

Reston, VA

$286860-0,001-0$

AMERICAN PARKING INC

Tulsa, $\mathrm{OK}$

$257480-0001-6$

AMERICAN PHYSICAL SOCIETY

College Park, MD

002880-0003-X

AMERICAN RUSSIAN ENV SERVICES

Dunedin, FL

$291220-0001-1$

AMERICAN SAMOA GOVERNMENT

Territarial Energy Office

Pago Pago, AS

AMERICAN SOC MECH ENGINEERS

Washington, DC

002960-0201-1

AMERICAN SPEC

$003020-0003-8$

Morgantown, WV

AMERICAN STATISTICAL ASSN

Denver, $\mathrm{CO}$

AMERICAN TEST CENTER

St Paul, MN

AMERITECH ENGINEERING INC

Corvallis, oR

AMSTUTZ DEAN E

Bloomitid, IA

ANAMEX CORP

Anaheim, CA

ANDERSON CONSTR CO

Pierre, SD

Waterlown, SD

ANDREW ROSS M

Columburs, $\mathrm{OH}$

ANGEL RAMIREX S

San Jusn, RQ

ANGUS ELECTRONIC

lindianapolis, IN

ANIXTER DENYER

Aurora, $\mathrm{CO}$

ANIXTER INC

Durham, NC

Norcross, GA

ANL \& ORNL

Oak Ridge, TN

ANTARES GROUP INC

Landover, MD

ANTEON CORP

Fairfax, VA
$289630-0001-X$

003050-0004-7

190900-0001-1

285588(t-000]-8

264090-0001-4

265080-0001-2

$278340-0002-0$

278340-0001-2

272690-0001-8

$277170-0001-6$

$284460-0001-6$

278300-0001-8

278310-0001-1

278310-0002-X

282980-0001-3

$289680-0001-8$

$286980-0001-0$
Awardee

Ideatification

code

AOK MEDICHAIR LTO

Deer Park, NY

$284510-0001-0$

APOCOM INC

Oak Ridge, TN

$252440-0001-2$

APPLIED QUALITY COMMUNICATION

Oxon Hill, MD

$286030-4001-8$

APPLIED RESEARCH ASSOC INC

Rakeigh, NC

APPLIED SCIENCE \& TECHNOLOGY

Wobum, MA

$159280-0003-0$

APPLIED TEST SYSTEMS INC

ButIet, PA.

$290960-0001-3$

APPLIX

Vienna, VA

APPROPRIATE TECHNOLOGY INTL

Washington, DC

285190-0001-0

APS TECHNOLOGY

Cromwell, CT

AQUARIIS ENGINEERING

Springdale, AR

ARCHITECTRE TECHNOLOGY CORP

Eden Prairic, MN

$289260.0001-\mathrm{X}$

ARETE ASSOC

Tucson, AZ

$288990-0001-8$

ARIZONA BOARD OF REGENTS

Tempe. AZ

265020-0001-0

ARIZONA CITY SANITATION SVC

Arizona City, AZ

ARIZONA STATE OF

Commerce Dept of

Phoenix, AZ

ARIZONA STATE UNIVERSITY

Chemistry \& biochemist Dept of Tempe, AZ

$286690-0001-6$

$004430-1001-3$

$004440-2501-4$

ARIZONA UNIVERSITY OF

Mining \& Geotopical Eng

Tucson, AZ

Plant Patholosy

Tucson, AZ

ARKANSAS STATE OF

AIDC Energy Ofilice Div

Liltle Rock, AR

County Operations Div of

Little Rock, AR

Soil \& Wrter Conserr

Little Rock, AR

ARKENOL HOLDINGS INC

Mission Viejo, CA

ARKENOL NNC

Mission Viejo, CA

ARLINGTON COMPUTER PRODUCT

Buffalo Grove, Il

$004450-3601-X$

$004450-2601.4$

$004510-0501-5$

$004510-0901-0$

$004510-1001-9$

$286200-0001-2$

260510-0001-1

ARM AUTOMATION

Austin, TX

290850-00OL-7

ARMENDARIZ CONSTRUCTION CO

Cimaaton, $\mathrm{CO}$

Craig, $\mathrm{CO}$

$70-0005$

$248670-0006-\mathrm{X}$ 


\section{Awardee}

ARMENTA RAY J

Los Alamos, NM

ARMOUR DRAY

Armour, \$D

ARMSTRONG COUNTY

Amarillo, TX

ARMSTRONG SANITATION

Williston, ND

ARMY DERARTMENT OF

Aberifeen, MD

Adelphi, MD

Picatinny Arscnal, NJ

Raleigh, NC

Wahiawa, $\mathrm{HH}$

Warren, MI

Albuquergue Resident Ofike

Kirtland, NM

Arkassas Army National Guard

Notb Little Rock, AR

Automotive Armanents Command Warien, Ml

Aviation \& Missile Conmand

Newpont News, VA

Aviation Techntical Test Ctr

Fort Rucker, AL

CECOM

Fort Monmouth, NJ

Communication/Electronics Comm

Mercury, NV

Corps of Engineers

Anchorage, AK

Little Rock, AR

Richland, WA

Seattle, WA

Walla Walla, WA

Winchester, VA

Corps of Engrs WWES

Vicksburg, MS

Industrial Ecology Center

Picatinny Arsenal, NJ

Oregon Army National Guard

Richland, WA

Public Works Dept of

Fort Hood, TX

Research Laboratory

Adelphi, MD

Structure Mechanics Div

Hamplon, VA

Water Resources Inst for

Fort Belvoir, VA

Waterways Experiment Station Vicksburg, MS

ARTHUR D LITTLE INC

Cambridge, MA

Cambridge, ME

AS ENGINEERING INC

Glendale, CA
Identification

code

$287570-0001-8$

291620-0001-2

$271180-0001-9$

291930-0001-4

004620-(KOS1-6

004620-0001-X

$004620-0082-6$

0. 4620-0053-2

004620-(X)B2-7

004620-0030-3

004620-A5B3-N

$004620-\mathrm{A}] \mathrm{AB}-\mathrm{N}$

$004620-A 430-B$

004620-A9B5-T

$004620-89 A 7-5$

$004620-9911-3$

004620-B0B6-M

004620-09A5-I

004620-09B1-F

004620-09A9-M

004620-09A4-H

004620-0961-0

004620-09A6-J

$004620-9829-x$

$004620-A 382-\mathrm{H}$

004620-A7A9-U

004620-A6B4-P

004620-A80]-D

004620-A2B0-H

(0)4620-A008-C

$004620-6629-0$

$004770-0002-9$

$004770-0005-3$

289870-0001-X
Awardet

Identification code

ASCENSION TECHNOLOGY

Waltham, MA

$285530-0001-X$

ASPEN EOUIPMENT

Bloomingtor, MN

$292990-000]-8$

ASPEN SYSTEMS CORP

Rockville, MD

ASSOC ARTS SCHOOL OF THE

St Paul, MN

$289940-0(x)]-1$

$172120-0001-9$

ASSOC RELOCATION MGMT CO INC

Washington, DC

197260-0021-1

ASSOC WESTERN UNIVERSITTES INC

Richland, WA

$005110-0002-2$

Salt Lake City, UT

$005110-0001-4$

AT \& T CORP

Alburquerque, NM

$260430-0002-4$

ATHENS MICRO COMPUTER CENTER

Elberton, GA

$286460-0001-\mathrm{X}$

ATKINSON INDUSTRIES INC

Pittsburg. KS

290620-0001-0

ATL INTERNATIONAL INC

Germantown, MD

285640-0001-6

ATLANTA UNIVERSITY

Chemistry Dept

Atlanta, GA

005290-0301-5

ATLANTIC MANAGEMENT CENTER INC

Falis Church. VA

$003300-0004-7$

Richland, WA

$003300-0005-5$

ATMOSPH UMWELT INSTITUTE OF

Skistrion West

Kreuzeckbahnstrasse, Georgia

269920-0101-9

AUBURN UNIVERSITY

Fotestry School of

Aubum, AL

$005530-0801-3$

AURORA ASSOC

Sarki Clara, CA

292480-0001-0

AUSTRALIAN TECH PRK SYDNEY ITD

Eveleigh, Australia

292290-0001-9

AUTOUDAD REG NUCLEAR

Buenos Aires, Argentina

$289810-0001-8$

AVCO BVERETT RES LAB INC Everet, MA

005810-0001-3

AVEKA INC

Woodbury, MN 289460-0001-5

AVENTEC DIRECT

Mentor, $\mathrm{OH}$

$2905060-0001-3$

AVERY DON E

Kaneohe, HI

$005830-0001-0$

AVERY S

Upton, $\mathrm{KY}$

$277970-0001-8$

AVERY SCHOOL DISTRICT $\$ 394$ Avery, ID

$283680-0001-7$

AVO INTERNATIONAL

Phoenix, AZ

$220970-0003-7$

AWK CONSULTING BNGINEERS INC

Turtle Creek, PA

$290740-0001-0$

AYL TECHNOLOGIES

Cerritos, CA

$283160-000]-6$ 


\section{Awardet \\ Identification \\ code}

B

B \& B CRANE \& EQUIPMENT INC Portland, $O R$

$179660-0002-8$

Redding, CA

B $\&$ H CONSTRUCTION INC

Watertown, SD

BABCOCK \& WLCOX CO

Alliance, OH

Lorain, $\mathrm{OH}$

Lynchburg, VA

Miamisburg, $\mathrm{OH}$

Research \& Development Div

Alliance, $\mathrm{OH}$

Lynchburg, VA

BACHAMP RICHARD E \& TONYA

Mashattan, KS

179660-0001-X

$275870-0001-1$

$005990-0001-5$

$005990-0007-4$

005990-0003-1

$005990-0009-0$

$005990-0601-3$

005990-0603-X

$272920-0001+0$

BAITELLE

Columbus, $\mathrm{OH}$

BALDING MARTIN

Ontario, CA

BANGERT THOMAS

Eureka Springs, AR

BANK OF AMERICA

San Francisco, CA

BANKS GARBAGE SERVICE

Fort Thompson, SD

BAPTIST HOSPITAL OF EAST TN

Admin

Kroxvilte, TN

288410-0001-5

$273140-0001-8$

$267560-0001-2$

$240700-0002-X$

291630-0001-6

BARANKO BROTHERS INC

Belfield, ND

BARLING POLICE DEPT Barling, AR

BARLOW ROBERT M Greal Falls, VA

BAROFF DR JAMES H Edgewater, MD

BARQUIN \& ASSOC INC Bethesda, MD

BARRETT MASON D Golden, $\mathbf{C O}$

BARTO ROSEMARIE Artington, VA

BASON COMPUTER INC Chatsworth, CA

$251720-0101-8$

$288310-0001-2$

283420-0001-X

$277500-0001-1$

$265190-0001-9$

$292950-0001-3$

283010-0001-5

$277780-0001-6$

BATTELLE COLUMBUS OPERATIONS Columbus, OH

288710-000]-3

BATTERY RESEARCH \& TESTING Oswego, NY

BATTRUSCH CONSTRUCTION CO INC Havre, MT

$264950-0001-4$

284070-0001-9

BAYFRONT MEDICAL CENTER S1 Petersburg, FL

$224680-0001-3$

BAZON COX \& ASSOC INC

Virginia Beach, VA

BC INTERNATIONAL

Hingham, MA
Awardee

Identification code

BDM INTERNATIONAL INC McLean, VA

BEACON LIGHT PRODUCTS INC

Meridian, ID

$287330-0001-8$

BECHTEL JACOBS CO LLC

Oak Ridge. TN

$276040-0001-0$

BECK ENGINEERING

Gig Hartor, WA

BECKMAN LEONARD

Elgin, NE

$287000-0001-9$

BECNEL GORDON

Luling, LA

BEHNE JR JOSEPH C Las Veggas, $N V$

BEEGER CARTAGE SERVICE Tulsa, OK

BELL ATLANTIC INC Washínglon, DC

BELL TELEPHONE CO Pittsburgh, PA

BEN \$MITH CONSULTING Columbia, TN

29219010001-6

$272240-8001-9$

$268310-0001-0$

$286610-0001-7$

$266730-000 \mathrm{j}-5$

$284980-0001-7$

$007380-00003-5$

BENCHMARK ENVIRONMENTAL CORP Albuguerque, NM

265310-0001-5

BENEWAH COMMUNITY HOSPITAL St Maries, ID

144140-0001-7

BENTLEY CO

San Ramon, CA

248940-0004-0

BENTON COUNTY OF

Sheriff's Office

Kennewick, WA

$007810-0201-\mathrm{X}$

BERKOWTTZ LESLIE Arlington, VA

BERKY RICHARD \& JERRY WAGNER Cedar Falls, [A

$277260-0001-5$

BERNALILLO COUNTY OF

Environmental Health Atbuquerque, NM

226850-0220]-4

GERRY LANE F Waddy, KY

BERTS TRUCK EQUIPMENT Chariton, IA.

BEST LOCKING SYSTEMS Santa Clarita, CA

BETA CORP INTERNATTONAL Washinglon, DC

271940-0001-6

$288180-0001-2$

287400-0001-X

246570-0002-0

BETH ISRAEL DEACONESS MED CTR

Radiological Services

Bosion, MA

$057930-0401-7$

BEVERIDGE DEGRANDI $W$ \& $Y$ Washington, $D C$

BIANCO JAMES H

Seaford, DE

$239310-0001-X$

BIEMENS

Vienna, VA

BINGHAM COUNTY OF

Blackfoot, ID
270480-0001-5

$266760-0001-6$

$169360-0001-0$ 
Identification
code

BIO-PROCESS INNOVATION INC

Dale Dr M Clark

West Lafayette, IN

$237410-0101-5$

BIODE INC

Bangor, ME

BIOINDUSTRIA

Georgelown, TX

BISON MOTOR CO

Grear Falls, MT

BLACKFOOT SCHOOL DIST $\$ 55$

Blackfoot, ID

BLACKSMLTH LARRY \& D METZGER

Burtinglon, IA

TECHNOLOGIES INC

221680-0002-6

289000-000]-2

BLAINE CO JOINT SCH DIST \#61 Hailey, ID

BLISS JOINT SCHOOL DIST \#234

Bliss, ID

$267030-0001-8$

BLUE SKY ENERGY \& ENV ENTERPR

Alexandria, VA

$287740-0001-2$

BLUERIDGE SOLUTIONS

Phoenix, AZ

BNFL INC

Oak Ridge, TN

BODINE ELECTRIC CO

Chicago, IL.

BOEING NORTH AMERICAN

Canoga Park, CA

Golden, $\mathrm{CO}$

BOFFIN LTD

Burnsville, MN

BOHLMANN JAMES $w$

Collinsville, OK

BOLCH MICHAEL E

Marietta, GA

BONER ALAN H

Eagene, OR

BONNER HAROLD W

Kirkwood, MO

BONNEVILLE POWER ADMIN

Richland, WA

Walla Walla, WA

BOSTON MICROSYSTEMS INC Wellesley, MA

BOSTON TRANSIT GROUP

Wakefield, MA

$293090-0001-5$

256580-0004-I

292520-0001-1

288780-0002-7

$288780-000+-9$

289730-0001-2

$270520-0001-6$

$292360-0001-0$

$268650-0001-3$

$268880-0001-X$

$261250-0002-8$

261250-0003-6

288900-0001-5

$290820-000]-6$

BOSTON UNTVERSITY

Public Health

Boston, MA

BOUNDARY CTY SCHOOL DIST \#101

Bonthers FerTy, ID

BOUNDLESS CORP

Boulder, $\mathrm{CO}$

BOWLING $\mathrm{M} \mathrm{C}$ Albany, GA

BOYER FORD TRUCKS

Minneapolis, $M N$

Sioux Falls, SD

$272700-0001-8$

$256500-0001-8$

$2565000-0002-6$
$292650-0001-5$

Awardee

Identification

code

BPF INC

Dukcanville, TX

BP]

Golden, CO

BPOI

Bakersfield, CA

BPW INC

DahJonega, GA

BRACO RESOURCES SERVTCES

Gardnerville, NV

Portand, OR

Sitka, AK

BRANDEGEE INC

Alexandria, VA

Pitsburgh, PA.

BRATCHER HAROLD

Seguin, TX

BRAZORIA COUNTY

Bryan Beach. TX

BREEK ED

Roanoke, TX

BRENNAN JIM

Filer, ID

BRIGGS DR JAMES D

Kerrville, TX

BRIGHAM YOUNG UNIVERSITY

Electrical \& Comp Eng Dept of

Provo, UT

254130-0002-2

$286580-0001 \cdot x$

$287200-0001-4$

$289470-0001-9$

258330-0004-1

258330-0005-X

258330-0003-3

$288600-0002-5$

288600-0001-7

$279620-0001-5$

$267720-0001]-3$

$265810-0001-9$

$270690-0001-4$

265180-0001-5

BRINDLESTONE CONSULTING SVCS Miamisburg. $\mathrm{OH}$

BRINK ELECTRIC CONSTRUCTION CO

Boulder Cily, NV

Devils Lake, ND

$132540-0029.3$

Greeley, $\mathrm{CO}$

BROADACRES NURSERY INC

Hubbard, OR

BROOKHAVEN SCIENCE ASSOC LLC

Upton, NY

BROOKHAVEN TECH GRP INC

Stony Brook, NY

$132540-0028-5$

293210-0001-1

$287190-0001-4$

285430-0001-7

BROWING JAMES A

Hanover, NH

BROWN JOHN ELSKE

Solana Beach, CA

$270370-000 \mathrm{~L}-9$

BROWN LEO $\mathrm{K}$

Idaho Falls, ID

HROWN PE MICHAEL A

$270790-0001-7$

$272230-0001-5$
Columbia, MD

BROWN UNIVERSITY

Psovidence, RI

BROWNING-FERRIS INDUST OF WV

Faimont, WV

BRUCE JOHN M

Mitchetl, SD

BRUDERIY ENGINEERING ASSOC

Gainesvilte, FL

010890-0002-2

BTAS INC

Mjamisburg, OH
239150-0002-7

270850-0001-5

$276090-000$ t -9

291190-000]-4
$273680-0001-6$ 
Identiflcation
code

BTG INC

Elverta, CA

257900-0003-7

Fainfax, VA

BURMEISTER ELECTRIC CO

Minneapolis, MN

BURRELLE'S NEWS EXPRESS

Livingston, NJ

BUS FOR SOCIAL RESPONSIAILITY

San Francisco, CA

257900-0004-5

$285820-0001-4$

287540-0001-7

BUSEK CO INC

Natick, MA

Needham, MA

Newton, MA

Research

Needhan, MA

BUSHMASTER FIREARMS

Portland, ME

BUSINESS OBJECTS AMERICAS

Bethesda, MD

BUSINESS OBJECTS INC

San Jose, CA

BUSINESS TECHNOLOGIES GROUP

Albuquerçue, NM

BUTTE COUNTY OF Arco, ID

BWC ENTERPRISES INC

Logandale, NV

BWX TECHNOLOGIES INC Lynchbutg, VA

BY.PRODUCTS CORP

Drexel Hill, PA

285780-0001-3

169900-0003-1

169900-0002-3

169900-000t-5

$169900-0102-x$

$284820-0001-2$

$291200-0001-4$

$250 \times 00-0003-4$

$291550-0007-0$

231370-0007-7

2858500-0001-5

286190-0001-2

284540-0001-1

C

C \& W ENTERPRISES INC

Denver, $\mathrm{CO}$

248480-0003-3

C F MULLEN INC

Seabrook, TX

280270-0001-7

CABE ASSOC

Dover, DE

CADDO DESIGN \& OFFICE DESIGN

Denver, $\infty$

$272170-0001-7$

CADMUS GROUP INC

Waltham, MA

$290810-0001-2$

$209020-0001-2$

CAHACON JOSE A

Albuquerque, NM

27 Jol0-0001-0

CAL POLY POMONA FOUNDATION

Center Regenerative Studies

Pomona, CA

CALABAZAS CREEK RESEARCH INC

Saratoga, CA

CALCPÓS ENGINEERING

Northfield Center. $\mathrm{OH}$

292270-0001-1

CALDWELL PHILLIP MACK

El Paso, TX

CALENERGY CO INC

Salton Sea Power Llc

Omaha, NE

$289650-0101-3$
Awardee

Identification code

CALENTINE DELLA D

Les Vegas, NM

$264 ! 90-0,001-7$

CALIFORNIA INSTITUTE OF TECH

Pasadena, CA

$012080-0001-2$

CALIFORNIA STATE OF

Enera Comm/Grants \& Lan Off

Secramen10, $\mathrm{CA}$

$012120-2502-4$

Energy Conmission

Sactamenio, CA

$012120-0702-6$

Energy Office

Sacramento, CA

012120-2302-1

Grants \& Loans Office

Sacramen10, CA

Health Services Department of

Berkeley, CA

Sactumenio, CA

Regional Water Quality Board

Sacramento, CA

CALIFORNIA UNIVERSITY OF

Molecular Cell \& Dev Bio Dept

Los Angeles, CA

0́12120-2602-0

012120-0301-2

$012120-0302-0$

$012120-2402-8$

Vice Chancellor Office of

Davis, CA

CALTRONICS BUSENESS SYSTEM

San Jose, CA

CAMAS COUNTY SCHOOL DIST \#121

Fairfield, ID

CAMBRIDGE JOINT SCH DLST \$432

Canbridge, ID

$283700-0001-0$

CAMBRIDGE UNIVERSITY OF

Cambridge, United Kingdot

$287910-0001-7$

CAMCO PAINTING SERVICE INC

Youngstown, $\mathrm{OH}$

259520-0001-0

CANADA CTR FOR REMOTE SENSING Oltawa, Canada

$264990-(x) 01-9$

CANCER RESEARCH INSTITUTE FOR

Fox Chase Cancer Center

Philadelphia, PA

$269930-0101-2$

CANDLE CORP

Santa Monica, CA

CAPITOL NORTH AMERICAN

Las Vegas. NV

CARLETON UNIVERSITY

Ohawa Ontario, Canada

CARNEGIE NaTURaL GaS Co

Pittsburgh, PA

CARNEGIE-MELLON UNIVERSITY

Mathematlcal Sciences

Pitssbugh, PA

$257330-0003-1$

$266770-(001-X$

$287820-0001-8$

$286470-0001-3$

CARTER CHEVROLET AGENCY INC

Okarche, OK

CARTER JOSEPH

Emmaus, PA

CASCADE SCHOOL DISTRICT \#422

Cascade, HD

CASE WESTERN RESERVE UNFV

Material \& Science Dept of

Cleveland, $\mathrm{OH}$
$278280-0001-4$

$013070-1201-9$

$012730.210]-0$

$245940-000]-0$

$71030-0001-8$ 
Identification
code

CASEWISE SYSTEMS INC

Waltham, MA

292500-0001-4

CATALYTICA COMBUSTION SYSTEMS

Mountain View, CA

289820-0001-1

CATERPILLAR INC

Mossville, IL

178220-0005-5

Engine Development

Peoria, IL

CCC \& ENGINEERS

Salt Lake City, UT

CCVD INC DBA MICROCOATING TECH

Chamblec, GA

$17 \$ 220-0201-5$

CD CONTRACTORS INC

McLean, VA

Vienpa, VA

CELERON GATHERING CORP

Long Beach, CA

CENTER FOR APPLIED RESEARCH

Denver, $\mathrm{CO}$

CENTRAL INTELLIGENCE AGENCY

Resion, VA

Finance \& Logistic Ofice of

Reston, VA

013970-0004X

$260210-00002-1$

$260210-0001-3$

166500-0003-5

289830-0001-5

CENTRAL KY COMM ACTION ENERGY

Lebanon, KY

281690-0001-7

CENTREX ELECTRICAL SUPPLY St Louis, MO

243300-0004-0

CENTURY COMPANIES INC

Miles City, MT

CERTIFIED SUBSTATION SERVICES

Duluth, MN

CERTIFIED TEST \& CONS SVCS

Lincoln, CA

$285900-0001-X$

166540-0003-X

CES/WAY INTERNATIONAL INC

Houston, TX

$286310-0001-9$

CETAC TECHNOLOGIES INC

Brian Oaks

Omaha, NE

287020-0001-6

CHALLENGES CHOICES \& IMAGE Englewood, $\mathrm{CO}$

$288160-0101-1$

CHALLIS JOINT SCHOOL DIST \#181

Challis, ID

$292080-0001 \cdot \mathrm{X}$

CHAMPION RESEARCH

Palo Alto, CA

CHAMPJON WIRE \& CABLE

Tempe, AZ

259280-0001-4

292210-0001-X

CHANEY DONALD

Pelersburg. AK

$292310-0001-2$

CHARLES KETTERING FOUNDATION

Yellow Springs, $\mathrm{OH}$

CHARLES M DELAJR JR COLLEGE

Modesto, CA

$264160-0001-6$

CHEMICAL INDUSTRY ENV TECH PRO

Columbus, $\mathrm{OH}$

CHEMMET LTD PC

West Richland, WA

CHEMMOTIF INC

Concord, MA
Awardee

Identification

code

CHERSKOV \& FLAYNIK

Chicago, lL

CHICAGO UNIVERSITY OF

Chicago, IL

Animal Resources Center

Chicago, IL.

CHILDRENS HOSPITAL CORP

Boston, MA

CHILDRENS HOSPITAL MED CTR

Cincinnati, $\mathrm{OH}$

CHILDS WILLARD

Encinilas, CA

CHOU CSS JAMES

Honolulu, HI

CHRISTENSEN RHYS

Ames, IA

CHROMATOFAST INC

Arn Artor, MJ

CHRYSLER CORP

Chicago, IL

CHUGACH ELECTRIC ASSOCIATION

Anchorage, AK

CIEMAT

Madrid, Spajn

CIMARRON SOETWARE

Salt Lake City, UT

CINCINNATI UNIVERSITY OF

Sponsored Programs Offlce of

Cincinnati, $\mathrm{OH}$

CINERGI CONSULTING PARTNERS

Golden, $\mathrm{CO}$

CISCO SYSTEMS INC

San Jose, CA

CLAREMONT GRADUATE UNIVERSITY

Claremont, $\mathrm{C}_{\mathrm{A}}$

Mathematics Dept

Claremont, $\mathrm{CA}$

CLARITY GROUP

Electotc Trans Application

Phoenix, AZ

CLARK ATLANTA UNIVERSTIY

Chemistry Department

Atlanta, GA

CLARK COUNTY OF

Dubois, ID

CLARK JOHN

Washington, DC

CLARK-MXR INC

Dexter, MI

CLAUSING INDUSTRIAL INC

Kalaniazoo, MJ

CLEAN AIR NOW

Santa Monica, CA

CLEAN FUELS STRATEGIES INC

Government Affairs

Artington, VA

CLEARY COLLEGE

Ypsilanti, MI
$287080-0001-8$

015130-0002-0

$015130-2102-8$

$121400-0001-3$

290230-0001-3

282300-0001-8

$270440-0001-0$

277220-0001-0

$284260-0001-0$

$015500-0004-7$

292300-0001-9

285170-0001-3

$284090-0001-6$

015630-1301-0

287160-0001-3

231590-0005-2

$110[90-0001-5$

$110190-0101-1$

259290-0102-2

$190950-060]-8$

015940-0003-4

270740-0001-9

$284270-0001-4$

264260-0001-9

$241480-0002-2$

$290750-0101-0$

282830-0001-2 
Identification
Awardee code

CLEAVELAND PRICE INC

Trafiord, PA.

CLEMSON UNIVERSITY

Anderson, SC

Geological Sciences

Clemson, SC

CLOUD N E

Winterville, GA

CNT-MICRO SYSTEMS

Pitısburgh, PA

COACTIVE AESTHETICS

Sausalito, CA

COALITION FOR SAFE ENERGY

Greenstrom, NC

COBLENTZ MICHAEL

Berkeley, CA

COEUR D'ALENE SCHOOL DIST \#2J1

Coeur D'Alene, ID

COLEMAN RESEARCH CORP

Washinglọ, DC.

COLLETTE JOHN

Fairbanks, AK

COLORADANS FOR CLEAN AIR

Denver, $C O$

245600-0001-3

$016240-0002-9$

$016240-1001-6$

$274530-0001-7$

$290680-090 \mathrm{t}-2$

$284370-0001-7$

$127190-0001-9$

$273690-0001-x$

279750-0001-9

224170-0007-5

$270750-0001-2$

COLORADO CENTER FOR ENVIR MGMT

Texh \& Regubtory Integration

Denver, $C O$

$211870-0102-3$

COLORADO NORTHWESTERN CMTY COL

Rangely, $\mathrm{CO}$

$017150-0001-3$

Natural Resonrces

Rangely, $\mathrm{CO}$

COLORADO STATE OF

Energy Conservation Office of

Denver, CO

Geological Surrey / Min \& Geol

Denver, $\mathrm{CO}$

Natural Resiources Dept

Golden, $\mathrm{CO}$

COLORADO STATE UNIVERSTYY

Boulder, $\mathrm{CO}$

COLUMBIA SERVICES GROUP INC

Fairfax, VA

COMARK FEDERAL SYSTEMS

Chartilly, VA

COMBUSTION RESOURCES ELC

Provo, IT

$017150-0101-x$

$017200-0801-9$

$017200-2001-9$

$017200-0505-2$

0]7210-0003-8

$202710-0004-X$

$290580-0001-x$

COMBUSTION SPECIALISTS INC

Maple Valley, WA

COMET GMBH PYROTECHNICK

Bremerhaven, Germany

COMMERCE DEPARTMENT OF

Las Vegas, NV

Census Bareas of the

Suitland, MD

Energy Admin

Lansing, MI

National Ocennic \& Atmos Admin Boulder, $\mathrm{CO}$

291690-0001-8

APPARA

$288000-0001-0$

$017570-0030-5$

$017570-0440-8$

$017570-4039-0$

0.7570-1203-6
Awardee

Identification code

Charleston, SC

$017570-3837 . \mathrm{X}$

Seattle, WA

$017570-1219-2$

Silver Spring. MD

$017570-1213-3$

Nat] Inst Standards \& Tech

Boulder, $C O$

017570-3903-1

COMMERCIAL ELECTRONICS INC

Broken Amow, OK

284100-0001-6

COMMERCIAL SIDING \& MAINT CO

Vernal, UT

290470.0001 .3

COMMUNICATIONS PRODUCTS INC

Indianazolis, IN

289370-0001-6

COMMUNICATIONS TRAINING ANAL

Arrington, VA

$287520-0001-X$

COMMUNITY REUSE ORGANIZATION

Largo, FL

COMMUTER CHECK CORP

Lake Lillian, MN

$240280-0002-5$

COMP USA INC

Emeryville, CA

$274830-0001-5$

Knoxville, TN

$240710-0003-1$

Vienna, VA.

240710-0005-8

240710-0004-X

COMPA INDUSTRIES INC

Albuquerque, NM

$2139500004-2$

COMPOSITE TECHNOLOGY DEV INC

Boulder, $C O$

159450-0001-9

Lafayette, $\infty$

$1594500002-7$

COMPREHENSIVE TECH INTL INC

Fairtax, VA

$194840-0002-9$

COMPRESSION DYNAMICS

Corry, PA

$289850-0001-2$

COMPUTER DATA SYSTEMS INC Aiken, SC

$214110.0001-3$

COMPUTER SALES INTERNATIONAL

St Clair Shores, MI

226170-0002-8

St Clair Shores, MO

$226170-0001-X$

COMPUTER SCIENCES CORP

Laurel, MD

COMPUTER SYSTEMS MGMT ENC

Alexandria, VA

018010-0005-0

COMPUWARE CORP

Farmington Hills, MI

$287660-0001-7$

COMTECH NET INC

018040-0003-5
Salt Lake City, UT

COMTEL TECH INC

Broonfield, $C O$

CONCEPT TECHNOLOGY

San Diego $+\mathrm{CA}$

CONCORD COMMUNICATIONS

Marlborough, MA

CONCURRENT TECHNOLOGIES CORP

Johnstown, PA

$287530-0001-3$

$279640-000 \mathrm{j}-2$

284350-000l-X

289380-0001-X

CONFERENCE OF ST LEGISLATURES

Denver, CO

CONNECTICUT \$TATE OF

Hartiond, CT

CONNECTICUT UNIVERSITY OF

SIors, CT

$018310-0008-3$

$018320-0002-8$ 
Awardee $\quad \begin{gathered}\text { Identification } \\ \text { code }\end{gathered}$

CONSERVATION TRUST OF PR

San Juan, PR

CONSOL INC

Library, PA

CONSOLIDATED GROUP OF TRIBES

Las Vegas, $\mathrm{NV}$

CONSORTIUM INC

Bethesda, MD

CONSTELLATI

Battimore, MD

CONSUMERS FEDERATION OF AMER

Whashington, $D C$

$263110-0001-6$

$261060-0002-6$

CONTAINERBOA Jesup, $0 \mathrm{~A}$

CONTRA COSTA COUNTY OF

Martinez, CA

CONTRACT ASSOC

Los Alarnos, NM

CONTROL CONCEPTS INC

Faizfax, VA

CONTROL DATA SYSTEMS

Arden Hills, MN

CONWEST GROUP INC

Phoentx, AZ

COOPERATIVE ADMIN SUPPORT UNIT

Denver, $\mathrm{CO}$

(1)-0002-1

COOPERATIVE DEVELOPMENT INST

Greenfeld, MA

COOPERS \& LYBRAND

Fairfax, VA

MeLean, VA

$018780-0016-0$

$265250-0001-3$

CORDOBA CORP

Sar Francisco, CA

$288260-0001-8$

COREL CORP

Orem, UT

$278250-0001-3$

CORNER CONSTRUCTION CORP

New Underwood, SD 289100-0001-5

CORONADO PAINT \& DECORATING

Los Alamos, NM

$291160-0001+3$

CORP FOR SOLAR TECH \& REN RES

Las Yegas, NV

CORPORATE INTELLIGENCE \& SEC

Clinton, TN

$252870-10001-4$

CORPUS CHRISTI CITY OF

Engineering Services

Corpus Christi, TX

$290920-0001-9$

CORRA JOHN P

Yardley, PA

CORRODOS INC

Bethesda, MD

COUNCIL ENERGY RESOURCE TRIBES

Denver, $\mathrm{CO}$

019100-0003-5

COUNCIL GREAT LAKES GOVERNORS

Chicago, IL

$019110-0002-0$

COUNCIL OF SCIEN SOC PRESIDENT

Washington, DC

COUNCIL SCHOOL DISTRICT \#I3

Council, ID

$283150-0001-2$

279700-0001-0
Awardee

Identlfication

code

COVENTRY TOWN OF

Coventry, CT

280760-0001-7

COVINGTON ST AREA VO-TECH SCH

Covington, 'IN $281730-0001-8$

CRAFTS INC

Апmон, SD 28589010001-X

CRANE JOHN

Camp Verde, AZ

$270760-0001-6$

CREATV MICROTECH INC

Polomac, MD

CREIGHTON \& CREIGHTON INC

Morgantown, WV

$288880-0001-1$

2075 10-0002-7

CREMAT

Watertown, MA

CRESCENT ELECTRIC SUPPLY CO

Fort Collins, $\mathrm{CO}$

290010-0001-0

CREST

Washington, DC

$286420-0001-5$

CRICHTON J L

Knoxville, TN

$284730-0001-3$

CRITIQUE RESOURCE CONSULTING

Golden, $\mathrm{CO}$

$270240-0001-5$

CRITIOUE RESOURCES CORP

New Otleans, LA

$261290-0002-2$

CROFT ROBERT E

Lovell, WY

$288080-0001-X$

CROMRICH JOHN

Clancy, MT

CROSBY GARDENS

Toledo, $\mathrm{OH}$

$277470-0001-4$

CROSS KENN

Blacksville, WV

CRYOGENIC \& VACUUM EQUIPMENT

Columbus, $\mathrm{OH}$

CRYSTAL SPRINGS BOTTLED WATER

Albuquergue, NM

$290800-0001-9$

CRYSTALS \& CERAMIC TECHNOLOGY

Fadianapolis, IN

$289170-0001-0$

CSI ENGINEERING PC

Laurel, MD

$263480-0002-8$

CSS LABORATORIES INC

Irvine, $\mathrm{CA}$

Information Mansgement Div

Irvine, CA

CTI INC

Fairfax, VA

$219050-0001-4$

219050-0101-0

CUBE CORP

McLean, VA

$259080-0002-7$

CULDESAC JOINT SCH DIST $\$ 342$

Culdesac, ID

292090-0001-3

CUNNINGHAM WALT

Port Bailey, AK

CUSTOM COALS INTERNATIONAI

Pittsburgh, PA

$283470-0001-8$

CYCLE SOFTWARE

Cambridge, MA

CYGNACOM SOLUTIONS INC

MicLean, VA
$281710-0001-0$

$225770-0002-2$

$292660-0001-9$

$287310-0001-0$ 
Identification
code

CZECH ACADEMY OF SCIENCE

Prague, Czech Republic

289530-0001-7

D

D \& R INTERNATIONAL LTD

Silver Spring, MD

$264930-0001-7$

D L MEACHAM CONSTRUCTION CO

Fteepon, TX

$288850-0001-0$

D R WHITE COMPUTER SVCS INC

Canton, MA

$286290-0001-5$

D'APPOLONIA CONSULTING ENGR

Pittsburgh, PA

020100-0001-3

DAFL KATHY

Winchester, KY

$271120-0001-7$

DAKOTA CLEANING SERVICE

Watertown, SD

DAKOTA ELECTRIC SUPPLY CO

Bismarck, ND

Fargo, ND

DAKOTA FENCE CO

Bismarck, ND

DALHOUSIE UNIVERSITY

Oceanography

Halifax, Canada

DALY COMPUTER INC

Gaithersburg, MD

DALY FEDERAL Gaithersburg, MD

DAMES \& MOORE Phoenix, AZ

DAN BOER ENG \& CONST CO Catbedral Cily, CA

DANKA OFFICE IMAGING Artington, VA

Rochester. NY

DANKO GERALD A

Powell, WY

DARDEN JOHN A

Lenox, GA

DARTMOUTH COLEEGE Hanover, $\mathrm{NH}$

DARTNELL ENTERPRISE Golden, $\infty$

DATA ASSOC

Fairfax Station, VA

DATA CARD

Tulsa, $\mathrm{OK}$

DATA GENERAL CORP Westborough, MA

DATA SOLUTIONS \& TECHNOLOGY Upper Marlboro, MD

DATA SYSTEMS MARKETING Upper Mariboto, MD

DATA TRANSIT INTERNATIONAL Denver, CO Golden, CO

DATACARD CORP

Bethesda, MD

284480-10002-1

284480-0001-3

291270-0001-X
Awardee

Identfficentfon

code

DAVIDSON \& ASSOC LLC

Miamisburg, $\mathrm{OH}$

DAVLS CONTRACTING CORP

Brentwood, MD

DAVIS KENDALL PC

Chicago, IL.

DAY DENNIS

Grimes, IA

DAY LAMAR

Bountiful, UT

DCS INC

Yardley, PA

DE LANOIT JR LEE

Fort Dodge, LA

DE LAUREAL ENGRS INC

New Orleans, LA

DEACON RESEARCH INC

Palo Alto, CA

DECISION STRATEGIES

Falls Church, VA

DECISION SYS TECHNOLOGIES INC

Rockville, MD

DEEP OCEAN ENGINEERING INC San Leandro, CA

DEEP SOLTH PECAN CO

Woodbury, GA

DEFENSE ANALYSES INSTMTUTE FOR

Arlington, VA

DEFENSE CIVIL PREPAREDNESS

Washinglon, VA

DEFENSE CONTRACT AUDIT AGENCY

Fort Belvoir, VA

DEFENSE CONTRACT MGMT COMMAND

Canoga Park, CA

DEPENSE DEPARTMENT OF

For Polk, LA

Philadelphia, PA

AF Aero Prop Lab

Wright-Patterson AFB, OH

Armed Forces Ins

Washingtol, DC

Aruy Air Force

San Antonio, TX

Defense Analysis Institate for Alexardria, VA

$283130-0001-5$

021190-0030-1

021190-0033-6

021190-3928-3

$021190-4304-3$

$021190-4029-\mathrm{X}$

$021190-5001-5$

Defense Nuclear

Kinland AFB, NM

Defense Personnel \& Supply Cir Philadelphia, PA

$021190-4733-2$

Defense Snpply Center

Columbus, $\mathrm{OH}$

Electromagnetic Comp Anal Ctr Annapolis, MD

National Reconnaissance Office

Washington, DC

National Security Agency

Fort Meade, MD
$021190-4632-8$

$02] 190-2002-7$

$021190-4404-X$

02]190-4935-1 
Idlentifieation
Awardee

Naval Postgradante

Monterey, CA

On Site Inspection Agency

Washington, DC

Special Operations Connuand

Macdill AFB, FL

$021190-4222-5$

$021190-5104-6$

$021190-5237.9$

Special Weapons Agency

Alexandria, VA

02 [ $190.4501-1$

Kirtland, NM

Tank Aatomotive Comnmand

Rock Island, IL

$021190-4536-4$

$021190-5338-3$

Usa Commannication Electronic Ealontown, NJ

$021190-4834-7$

DEFENSE FINANCE ACCOUNTING SVC Bellevue, NE

DEFENSE INDUSTRLAL SUPPLY CTR

Philadelphia, PA

$288380-0001-8$

DFENSE INFO SYSTEMS AGENCY

Guntersville, Al

DEFENSE INTELLIGENCE AGENCY

Wamer Robins, GA

$291250-0001-2$

$228280-0006-0$

155340-0003-1

DEFENSE LOGISTICS AGENCK

Columbus, $\mathrm{OH}$

$021200-0006-9$

DEFENSE SYSTEMS MGMT COLLEGE

Fort Belvoir, VA

288540-0001-9

DEFENSE THREAT REDUCTION AGCY Albuquerque, NM

292230-0001-7

DEFTANCE CITY SCHOOLS

Defiance, $\mathrm{OH}$

DEFRANCESCO MICHAEL P Exeter, RI

$264140-0001-9$

$273720-0001-7$

DEKRAFFT III JAMES A

Richmond, VA

$270380-0001-2$

DEL MONT CONSULTANTS INC

Dove Creak, $C O$

$244530-0002-2$

$244530-0004.9$

$244530-0001-4$

244530-0005-7

$244530-0003-0$

Shiprock, NM

$264 \times 50-0001-2$

Del Norte, $C O$

DEL-SIR SUPPLY INC

Pittsburgh, PA

$285 \mathrm{t} 80-0001-7$

DELAWARE COUNTY OF

Intermediate Unit

Broomal], PA

$021330-0103-4$

DELAWARE PUBLIC SERVICE COMM Dover, DE

$264170-0001-X$

DELAWARE STATE HOSPTTAL

Health \& Soctal Sves Dept

New Castle, DE

021370-010]-2

DELAWARE STATE OF

Corrections Departiment of

Wilmington, DE

Energy of Delaware

Dover, DE

Faciltities Management Div of Dower, DE

$021380-1102-X$

$021380-1001-5$

$021380-1301-4$
Awrardee

Identification code

Manageneut Budget Panning

Dover, DE

$021380-1201-8$

DELAWARE TECH \& CMTY COLL

Sonthern Campus

Georgetown, DE

DELCO ELECTRONIC

Goleta, CA

$021390-0402-7$

DELL COMPUTER CORP

Round Rock. TX

DELL MARKETING CORP

Round Rock, TX

Limited Partnership

Golden, $\mathbf{C O}$

$264180-000 \mathrm{~d}-3$

$2+7250-0005-9$

$247320-0003-7$

DELMARVA POWER \& LIGHT CO

Wilmington. DE

$24732040102-5$

DELMONT CONSULTANTS INC Shiprock, NM

Vernal, UT

DELOTTTE \& TOUCHE

Washington, DC

DELTA SOLAR SYSTEMS

Blytheville, AR

DELTA STAR INC

San Carlos, CA

DELTA VOTECH SCHOOL Marked Tree, AR

DEMAYO DEN \& MICHAEL BLYTH

Carrollton, GA

DEMSTER STANL.EY M

Midlothian, VA

DENCO MANUPACTURING CO Springfield, MO

DENDOOVEN K C

Las Vegas, NV

228980-0002-7

$183120-0002-\mathrm{X}$

$183120-0003-8$

$196470-0003-0$

$264200-0001-7$

$180050-0004.9$

$264210-0001-0$

$264230-000]-8$

$264220-0001-4$

$264240-0101-8$

$271060-10001-9$

DENVER UNIVERSITY OF

Colorado Seminar

Denver, $\mathrm{CO}$

DENVER WATER BOARD

Denver, $\mathrm{CO}$

Golden, $\mathrm{CO}$

DESCON

Emervijle, CA

DESERT EAGLE SECURITY

Phoenix, AZ

DESERT HOT SPRINGS CITY

Desert Hod Springs, CA

DESERT RECLAIMLTION IND

Plainfield, NJ

DESERT WEED CONTROL INC Arlington, AZ

DESIGN ALTERNATIVES INC Weshinglon, DC

DESIGN STORAGE SYSTEMS Auburn, CA

$0216401001-9$

261770-0001-0

261770-0002-9

$264320-000 t-7$

$284400-000 \mathrm{t}-4$

$264330-0001-0$

$264340-0001-4$

$288330-0004 \cdot x$

$264350-0001-8$
DETROIT UNIVERSITY OF

Detroit, $\mathrm{Ml}$

DEVELOPMENT \& RESOURCE TRANS

Silver \$pring, MD
$286010-0001-0$

$021930-0001.8$

$264360-0001-1$ 
Awardee Identification

DEVELOPMENT PLNG \& RES ASSOC

Manhattan, KS

021950-000]-5

DEVELOPMENT SCIENCES INC

East Sandwich, MA

Jenkintown, PA

Sagamore, MA

DEVMAR CONSTRUCTION

Bethesda, MD

DEWBERRY NEALON \& DAVIS

Pulaski, VA

DEYARMAN KERRY

Cedar Rapids, LA

DHL WORLDWIDE COURIER

Arlingion, VA

$000200-0002-2$

000200-0001-4

$000200-0003-0$

264370-0101-1

264380-00001-9

$285290-0001-3$

DIALOG CORP

Mountain View, CA

287940-0001-8

DIAMOND BACK SERYICES CORP

Shiprock, NM

DIAMOND DETECTIVE AGENCY INC

Hazel Crest, IL

DLAMOND OIL WELL DRILLING CO Midland, TX

DIAMOND VALLEY FIRE DEPT

Euteka, NV

DIAMOND WEST ENERGY CORP

Salı Lake City, UT

DICKERT P M

Augusta, GA

DILAKE INC

Manassas, VA

DIE. MESH CORP

Pelham, NY

DIEDE CONSTRUCTION INC

Woodbridge, CA

DHETER BRYCE INC

Gaston, SC

DIETRICH SCHOOL DISTRICT \#314

Djetrich, ID

DIGILOG INC

Broomfield, CO

Montgomery. PA

DIGITAL EQUIPMENT CORP

Alpharetta, $G \mathrm{~A}$

Golden, $C O$

Merrimack, NH

DIGITAL INK DESIGN

Las Vegas, NV

DILLCO INC

Plaçuetrine, LA

DILLINGHAM CITY \& COUNTY

Public Library

Dillingham, AK

DILLINGHAM CORP

$D \& C$ Co

Honolulu, HI

DILLINGHAM SCHOOL DISTRICT

Strict Carol Cherry

Dillingham, AK

$264560-0101-3$

$264440-0001-7$

205330-0002-5

$264450-0001-0$

283030-0001-2

$289670-0001-4$

$283640-0005-2$

095760-0004-1

$095760-0003-3$

$022160-0030-2$

$022160-0001-9$

$022160-0029-9$

$283720-0001-8$

$106630-0002-5$

264540-0101-6

$264550-0] 01-X$
293040-0001-7

Awardee

Identification code

DILLINGHAM SCHOOLS LIBRAKY

Dillingham, AK

$264570-0001-0$

DIMETRODEN

Warren, VT

$264580-000]-4$

DINGLE ASSOC INC

Washington, DC

DIRECTED TECANOLOGIES INC Artington, VA

DISTRICT OF COLUMBIA

Washington, DC

Administrative Services Dept

Washington, DC

Corrections Dept

Lortun, VA.

Rockville, MD

$022180-0001-6$

$284740-0001-7$

Energy Offlce

Washington, DC

Environmental Sres Dept

Washington, DC

$022330-0003-X$

$022330-0703-4$

022330-0201-6

$022330-0202-4$

$022330-0603-8$

Housing \& Cmty Dev Dept

Washington, DC

$022330-0303-9$

Flanning Olike

Washington, DC

Public Service Commission

Washington, DC

DITECH

Cape Gírardeat, MO

DIVERSIFLED FIELD \& LAND SVCS

Yakima, WA

022330-0503-1

$022330-0303-0$

022330-0903-7

2646]0-0001-1

DIVERSIF]ED TECHNOLOGIES INC Bedford, MA

Lexington, $\mathrm{MA}$

DIXIE FIBERGLASS PRODUCTS INC

Winter Garden, FL

DOANE COLLEGE

Crete, NE

DOBLE ENGINEERING CO

Waterville, WA

$162510-0002-3$

C

192480-0002-9

192480-0001-0

DOMNING BERNARD

Mobile, AL

DONALD CLARK ASSOC

Boulder City, NV

$264660-0001-X$

$264670-0001-3$

$022450-0004-8$

$264580-0001-7$

DONALD GaLLINO INC Redding, CA

$022640-0004 x$

$28635000001-3$

DONALDSON JESSE C

Cocosa, FL

$2647200001-8$

DONNELLEY MARKETING

Federal Marketjig

Stamford, CT

$264730-0101-8$

DOROTHY JEMISON FOUNDATION

Golder, $\mathrm{CO}$

Houston, TX

DOSCO CORP

Pittsburgh, PA

DOSHI A ASSOC INC

Appleton, WI

$2849600002-8$

$284960-0001-\mathrm{X}$

$264760-0001-2$

DOUBLE $\mathrm{L}$ MFG INC American Falls, ID
284430-0001-5

264780-0001-X 
Awardee $\begin{gathered}\text { Identlfication } \\ \text { code }\end{gathered}$

DOUGLAS SCHOOL DISTRICT

Rapid City, SD

DOVER CITY OF

Dover, $\mathrm{OH}$

DOWNTOWNER RETIREMENT APTS

Great Falls, MT

DRAIN CITY OF

Drain, OR

DRAKE UNIVERSITY Des Moines, IA

DRAVO CORP

Pitlstbugh, PA

DRESSER INDUSTRIES INC

Muskegon, MI

Jeffery Mining Machinery

Columbus, $\mathrm{OH}$

DREW-IT CORP

Hampion, NH

DRI MCGRAW HILL INC Washingtor, DC

DRION RESEARCH INC Cannbridge, MA

DROOKER MICHAEL S

Sanbornville, NH

DRS OF OAK RADGE LLC Oak Ridgt, TN

DRY CREEK RANCHERIA Healdsburg, CA

DRYERMATE CO Xenia, OH

DSS ENGINEERS INC Fort Lauderdale, FL

DU PONT CO Wilmington, DE

DUBIN-BLOOME ASSOC New York, NY

265130-0001-7

$155400-0002-i$

$265140-0001-0$

$265370-000 \mathrm{~L}-7$

$232050-0001-3$

022970-0002-2

022990-0002-X

$022990-020]-4$

$265330-00001-0$

191900-000]-3

265390-0001-4

273740-000]-4

286740-0001-0

$265400-0001-4$

265410-0001-8

265420-0001-1

265430-0000]-5

265450-0001-2

DUCK VALLEY INDLAN RESERVATION Owybee, NV

DUECO INC Waleriown, SD

DUERR DUANE DAVID Aspers, PA

DUFFY ROSS \& CAROLYN Topeka, KS

$265460-0101-2$

266700-0001-4

$265440-0001-9$

DUKE COGEMA STONE\& WEBSTER LLC Charlotte, NC

DUKE ENGINEERING \& SERVICES Austin, TX Charlotte, NC

DUKE SOLUTIONS Charlote, NC

DUKE UNIVERSITY Durham, NC

Environguentad Wedand Center Durham, NC

DUKERT JOSEPH Washington, DC
Awardee

Identtfication code

DULUTH YWCA

Doluth, MN

265490-0001-7

DUNCAN LAURA S

Austin, TX

DUNN \& BRADSTREET

Fafls Church, VA

DUPONT LANXIDE COMPOSTTES

Newark, DE

DURA CRANE DNC

Anderson, $\mathrm{CA}$

DURACRANE

Redding, CA

DURAND JAMES

Hartsburg, MO

DURHAM CITY OF

Durtham, NC

DUTRO CO

Emeryville, CA

DUTTON NELSON W

Liberal, KS

DWIGHT CORRECTIONAL CENTER

Dwight, IL

DWYER-MERCER DISTR

ICT Library

Celina, OH

DXVE INC

St Genevieve, MO

DYLlaCCO THOMAS E Highland, of

DYNAFLOW INC

Fulton, MD

DYNAMIC FLOW INC Morgantown, WV

DYNATECH R \& D CORP

Cambridge, MA

DYNCORP

Germantown, MD

DYNCORP EYNSP INC

Alexandria, VA

DynMeridian

Alexandria, VA

$272010-0001-2$

267730-0001-7

219060-0001-8

$286760-0001-8$

290640-0001-8

$270460-0001-8$

265500-0001-7

$265510-0001-0$

$265520-0000 \mathrm{~L}-4$

265530-0001-8

265540-0101-8

$266930-000 \mathrm{~L}-0$

265550-0001-5

288690-0001-X

265560-0001-9

$023650-0001-0$

$258890-0002-0$

$286900-000 t-1$

286900-0101.8

E

E E GOLDEN CO

Wheeling, WV

E H WHITE \& CO

San Francisco, CA

E I DUPONT DE NEMOURS \& CO INC

IETY Chem Dept

Wilmington, DE

Petrolean Laboratory

Wimington, $D E$

E R JOHNSON ASSOC INC

Vienna, VA

E-TECH INC

Atlania, GA

E2 SOLUTIONS

Golden, $\mathrm{CO}$
$265570-(000) 1-2$

$265580-000 t-6$

$023370-0102-4$

023370-0202-0

$023800-0003.4$

$208750-0001-0$

287320-0001-4 
Awardee $\begin{gathered}\text { Identification } \\ \text { code }\end{gathered}$

EA TECHNOLOGIES LTD

Capenhurst Chester, United Kingdom254270-0002-X

London, United Kingdom 2\$4270-0001-1

EAC

Nantucket, MA

$289390-0001-3$

EAGLE LIBRARY

Eagle, AK

EAGLE PICHER INDUSTRIES INC

Joplin, MO

Miami, OK

Quapaw, OK

Electronics Div

Mianti, OK

EARTH CONSERVATION CORPS Pendieton, OR

EARTH DAY NEW YORK INC New York, NY

EARTH INT LIVING SYS

Winters, CA

$2656600-0001-1$

EARTH POWER PRODUCTION CO

Eastern Technology Div

Los Angeles, CA

EAST HARHOR VILLAGE CENTER INC

Baltimore, MD

023880-0003-3

$023880-0001-7$

$023880-0002-5$

023830-0301-6

242950-0002.7

$287930-0001-4$

$265700-0001-2$

EAST ISLIP SCHOOL DISTRICT

Education Board of

East Islip, NY

EAST MACHIAS TOWN OF East Machias, ME

EAST TN MATERIALS \& ENERGY CRP

Oak Ridge, TN

EAST WTND COMMUNITY

Tecumseh, MO

EASTERN DATA PRODUCTS Middletown, MD

EASTERN INSTRUMENTATION

Moorestown, NJ

EATON INDUSTRIES OF HOUSTON

Houston, TX

EC CORP

Fernald, OH

Gaithersburg, MD

Oak Ridge, TN

ECHELON CORP

Pajo Alto, CA

ECI SYSTEMS \& ENGINEERING

Albuquerque, NM

Bronxville, NY

Castle Point, NY

Cheyenne, WY

Grand Junction, CO

Virginia Beach, VA

ECKBO DEAN AUSTIN WILLLAMS

San Francisco, CA

ECKERLIN HERBERT M

Raleigh, NC

ECLIPSE TECHNOLOGY INC

Oak Ridge, TN

$265730-000[-3$

$024330-0002-5$

$284630-0001-0$

100740-0001-2

024630-0003-1

$024630-0002-3$

024630-0001-5

284390-0001-4

286050-0002-3

286050-0005-8

$286050-00006-6$

286050-0003-1

$286050-0004-\mathrm{X}$

$286050-0001-5$

265740-0001-7

$269090-0001-3$

$291350-0001-5$
Awardee

Identification code

ECO NW

Eugene, OR

$265750-00001-0$

ECO-CONTROL INC

Cambridge, MA

$265760-000]-4$

ECOENERGETICS INC

Vallejo, CA

024670-0002-8

ECOLOGY \& ENVIRONMENTAL INC

Buffillo Corporate Center

Idaho Falts, ID

024680-0105-2

ECOLOGY CENTER INC

Berkeley, CA

265780-0001-1

ECOLOGY INST OF

Indianapolis, iN

$265790-000[-5$

Holcomb Research Inst

Indianapolis, IN

$2657900101-1$

ECOLOGY SCIENCES

Vienna, VA

$265770-0001-8$

ECONERGY ASSOC

New York, NY

ECONERGY INTERNATIONAL CORP

Boulder, $\mathrm{CO}$

ECONOMY MAINTENANCE SUPPAY

Fairfax Stabion, VA

$168230-0002-3$

ECOSYSTEMS STUDIES INST OF Millbrook, NY

$267770-0001-1$

ECOTECH

Sykesville, MD

$288560-0001-6$

ECP CONSULTING INC

Grand Junction, $C O$

ECUMENICAL SOCIAL ACTION COMM

Jamaica Plain, MA

265800-0001-5
EDGEMARK

Fairfield, $\mathrm{OH}$

EDGEMONT SCHOOL DISTRICT

Edgemont, SD

EDISON MATERIALS TECH CENTER

Dayton, $\mathrm{OH}$

EDLOW INTERNATIONAL CO

Washington, DC

$290570-0001-6$

EDMONSON COU

Brownsville, KY

EDS FEDERAL CORP

HeTniton, VA

EDSEL B FORD INSTITUTE

Detroib Ml

EDUCATION COMM OF THE STATES

Denver, $\mathrm{CO}$

Edacation Programs Div

Denver, $\mathrm{CO}$

EDUCATION FOUNDATION OF NY

New York, NY

EDUCATIONAL ABC OF AMER INDUS

Niagara Falls, NY

EDUCAMONAL FACILITIES LAB

New York, NY

CDUCATIONAL INSTITUTE OHOLEI T

New York, NY
265880-0001-4

$292250-0001-4$

$25341040003 . \mathrm{X}$

$2658700001 \cdot 0$

024920-0006-0

267290-0001-5

$024930-000 \mathrm{l}-3$

$024930-0101-X$

265890-0001-8

265900-0001-8

265910-0001-1 
Awardee $\begin{gathered}\text { Identification } \\ \text { code }\end{gathered}$

EDUTEL COMMUNICATIONS INC

Palo Alto, CA

EDWARD A ZAWADZKI LTD

McMurray, PA

$265920-0001-5$

265930-0001-9

EDWAROS R O

Lexington, $\mathrm{KY}$

$277130-0001-1$

EG \& G INC

Las Vegas, NV

025080-0001-9

Waltham, MA

EG \& G MOUND APPLIED TECH

Miannisburg, OH

$025080-0012-4$

$176610-0001-6$

EG \& GERVICES

Manassas, VA

EG \& G WASC INC

Albuquerque, NM

Gaithersbuzg, MD

EI REVIEW \& CO INC

Knoxville, TN

EIC INC

New York, NY

Newton, MA

EICKOFF CONSTRUCTION CO

Crooksion, MN

EIKONLX CORP

Burlington, MA

EIMCO TUNNELING \& MINING Salt Lake City, UT

ELECTRIC BOILER CORP OF AMER Aclantic, IA

$265980-0001-7$

ELECTRIC FUEL PROPULSION

Rockcor Inc

Redmond, WA

RLECTRIC PASSENGER CARS

San Diego, CA

ELECTRIC VEHICLE ASSOC

Beltsville, MD

Cleveland, $\mathrm{OH}$

ELECTRIC VEHICLE ASSOC OF AMER

Saj Francisco, CA

$265990-0101-7$

$266000-0001-5$

266010-0001-9

266010-0002-7

283320-0001-7

ELECTRICAL \& ELECT ENG INST OF

New York, NY

039970-0001-8

Piscalaway. NJ

$039970-0002-6$

Wasshington, DC

Nuclear \& Ylasma Sci Society

Piscalaway, N\}

039970-0003-4

Physics Dept

New York, NY

$039970-0302-5$

Tech Activities Dept

New York, NY

ELECTRO MAGNETIC APPLICATIONS

Albuquetque, $\mathrm{NM}$

ELECTRO TEST \& MAINTENANCE

Rapid City, SD

$286430-000 t-9$

ELECTRO TEST INC

Denver, $\mathrm{CO}$

$293000-0001-2$

ELECTROLYSER CORP

Toronto, Canada

$283460-0001-4$
Awardee

Identification

code

ELECTRONIC DATA SYSTEMS CORP

Germantown, MD

J78680-0003-1

ELECTRONIC FLOW METERS INC

Garland, TX

$266030-0001-6$

ELECTROSOURCE INC

San Marcos. TX

$290210-0001-6$

ELIM COMMUNITY LIBRARY Elim, AK

$266040-0101-6$

ELKTON PUBLIC SD $\$ 5-3$

Elkton, SD

$266050 \cdot 0001-3$

ELLEY H D

Jamestown, MO

ELLIS GEORGE \$ Chardon, $\mathrm{OH}$

ELRICK \& LAVIDGE

Chicago, IL

ELWELL CHRISTIAN \& WILL Conway, MA

EMC CORP

Bethesda, MD

$266060-0001-7$

$268000-0001-9$

$266080-0001.4$

$266090-0001-8$

$110310-0003-8$

EMC INC

Memphis, TN

EME GROUP

Alexandria, VA

$266100-0001-8$

Berlin., NH

245150.0008-X

$2451504017-9$

245150-0012-8

$245150-0014-4$

$245150-0011-\mathrm{X}$

245150-0007-1

$245150-0009-8$

245150-0018-7

$245150-0016-0$

245150-0019-5

245150-0015-2

$245150-0010-1$

245 I50-0013-6

Wilkes-Bante Township, PA

ATORS

EMERGENCY
Augusla, GA

EMMA PENDLETON BRADLEY HOSP

East Providence, RI

$266110-0001-1$

EMR

Alexandria, VA

ENCOTECH INC

Schenectady, NY

$283360-0001 \cdot 1$

ENCOTH CORP

Princetion, NJ

ENDUREX

Dallas, TX

ENERFEX INC

Burlington, VT

ENERGAS CO

Dallas, TX

ENERGEN INC

Bedford, MA

266120-0001-5

$266130-0001-9$

$266140-0001-2$

$291700-0001-8$

065350-0003-6

$285110-0001-1$

ENERGETICS INC

Dallas, TX

Washington, DC
$025930-0004-\mathrm{X}$

025930-0005-8 
Identification
code

ENERGETICS MARKET \& MGMT ASSOC Mountain Home, $\mathrm{DD}$ 266160-0001-X

ENERGY \& ENV RESEARCH CORP Irvine. CA $025980-0001-3$

ENERGY APPLICATIONS SYS INC Cartsbad, CA Del Mar, CA $106730-0003-6$ $106730-0002-8$

Paclfe Sierra Research Rancho La Costa, CA

$106730-0101-6$

ENERGY ASSOC Concord, MA

ENERGY ASSOCLATION Washington, DC 266: 70-0001-3

ENERGY AUDTTORS \& CONSULTANTS Highlands, NJ'

ENERGY BIOSYSTEMS CORP The Woodlands, TX 245080-0008-8

ENERGY CENTER OF WISCONSIN Madison, WI

$283140-0001-9$

ENERGY DECISIONS INC

Washington, DC

$285920-0001-7$

ENERGY DEPARTMENT OF

Conservation

Oklahoma City, OK

Environment, Safety \& Health

Washington, DC

Fossil Energy Office of

Washington, DC

Golden Field Office Golden, $C O$

Oakland Operations Office Oakiand, CA

Richland Operations Office Richland, WA

266190000010

Western Area Power Adnum Folsom, CA

$026050 \cdot 2528-4$

$026050-2621-3$

$026050-2721 \cdot \mathrm{X}$

026050-2909-3

$026050-2805-4$

$026050-0811+8$

Yucca Mountain SC Office Las Vegas, NV

$026050-0730-8$

ENERGY DEVE

026050-3029-6 Hazen, ND

ENERGY EDUCATION INST Canby, MN

ENERGY EDUCATION PROGRAMS

Woodstock, $\mathbb{L}$

ENERGY EFFICIENT BLDG ASSN Minneapolis, MN

ENERGY EFETCIENT PROCUREMENT Ohto Office of Direct Service

Columbus, $\mathrm{OH}$

$285620-0101-5$

ENERGY ENVIRON ANALYSIS Arlington, VA

ENERGY ENVIRONMENTAL ASSOC Arlinglon, VA $266230-0001-1$

ENERGY FOR THE EIGHTIES FDN Washington, DC

$266240-000]-5$

ENERGY INC ldaho Falls, ID

266150-0001-6

$154260-0001-8$
Awardee

Identification code

ENERGY INNOVENTIONS

Lincoln, NE

ENERGY INTERFACE ASSOC

Los Angeles, CA

ENERGY INVESTMENT INC

Baltimore, MD

Columbus, $\mathrm{OH}$

Indianapolis, IN

Los Angeles, CA

Pittsburgh, PA

Sitver Spring, MD

ENERGY MANAGEMENT SERVTCES INC

Santa $\mathrm{Fe}, \mathrm{NM}$

ENERGY MASTERS CORP

St Paul, MN

(60270001-6

145080-0002-9

ENERGY PACIFIC LLC

Los Angeles, CA

288960-0001-7

ENERGY PERFORMANCE SVCS INC

King of Pnassia, PA

$291780-0001-7$

ENERGY POLICY STUDIES INC

Planning Analysis \& Evahuation

El Paso, TX

ENERGY RESEARCH \& DEV ADMIN

Dak Ridge, TN

ENERGY RESEARCH CO

New York, NY

ENERGY RESOURCES OFFICE OF Augusta, ME

ENERGY SYSTEMS ASSOC INC

Pittspurgh, PA

244950-0016-0

244950-0021-7

244950-0017-9

244950-0020-9

244950-0019-5

244950-0008- $\mathrm{X}$

NERGY TECHNOLOGY INC

Cleveland, $\mathrm{OH}$

ENERGY TECHNOLOGY SUPPORT UNIT

England, United Kíngdon

ENERGY UNLJMHTED INC

Lincoln, MA

ENGAGE ENERGY US

Houston, TX

$266280-0101.6$

226720-0002-6

$254520-01002 \cdot \mathrm{X}$

266290-0001-3

$279430-0001-3$

266300-000]-3

$154270-0002-\mathrm{X}$

$266330-0001-7$

ENGTNEERED DATA PRODUCTS INC

Chesapeake Beach, MD

$285800-0001-7$

ENGINEERING APPLICATIONS INC

Rolla MO

ENGINEERING MGMT \& DEV

San Diego, CA

$266320-0001.0$

ENGINEERING RES CONSULTING INC

Lamont, $O K$

ENGINEERS INTERNATIONAL INC

Downers Grove, IL

Westrnont, IL

ENGINEERS RESEARCH \& ED TRUST

Jolint Labor-Mgmt Trust Fund

Washington, DC

ENGLESIDE MOTEL

Beach Haven, NJ

283380-0101-5

ENGLEWOOD HOS

$270830-000:-8$

Englewood, NJ

ENOSBURG FALLS SCHOOL DIST

Enosburg, VT
$100900-0002-1$
$288320-000 \mathrm{~J}-6$ 

Awardee
Identification code

ENOVA ENERGY INC

San Diego, CA

280360-0001-6

ENRON CAPITAL \& TRADE RESOURCE

Golden, $\mathrm{CO}$

ENRON TEAM

San Ramon, CA

$24 \$ 440-0002-0$

ENSCI INC

College Park, MD

$291790-0001-0$

ENSOURCE

$266340-0001-8$

Los Angeles, CA.

$285790-0001-7$

ENTE PER LE NUOVE TECHNOLOGIE

Rome, lialy

ENTELCO CORP

Webstrand Div

Toledo, OH

292120-0001-0

ENTER SOFTWARE INC

Menlo Park, CA

ENVIR \& LIFE SUPPORT TECH INC

Lakewood, $\mathrm{CO}$

ENVIRO-MANAGEMENT \& RESEARCH

Chesapeake, VA

ENVIROGENIC SYSTEMS

El Monte, CA

ENVIRONMENTAL

Berkeley, CA

ENVIRONMENTAL FUTURES

Boston, MA

$266350-0101-8$

291990-0001-6

284120-0001-3

254360-0002-9

$266370-0001-9$

266380-0001-2

ENVIRONMENTAL PROTECTION AGCY

San Francisco, CA

Washington, DC

Contracts \& Grants Ofifice of

Silver Spring, MD

ENVIRONMENTAL RES INST OF MICH Arn Arbor, MI

026690-0001-0

Reiations

Ans Artor, MI

ENVIRONMENTAL RES INST STATES

Washington, DC

026690-0101-7

ENVIRONMENTA

291310-0001-0

Grange, CA

ENVIRONMENTAL SCHOOL OF Rolnnert Park, CA

266390-0001-6

ENVIRONMENTAL \$Cl \& TECH FDN

Richland, WA

ENVIRONMENTAL SCIENTISTS INC

Gofrstown, NH

026670-0020-X

026670-0005-6

026670-2619-5

ENVIRONMENTAL TECH \& EDUCN CTR

A.buquerque, NM

284850-0001-3

ENVIRONMENTAL THERMOGRAPHY

Hilton Hcad Island, SC

$266410-0001-\mathrm{X}$

ENVIROSCIENCE INC

Hot Springs, AR

$290080-0001-6$

ENVIROSPHERE CO

Beflevite, WA

179420-0002-8

ENVIROTECH WASTE SERVICES

Pierre, SD

ENVISION ASSOC

Rochester, $\mathrm{NY}$
Awardee

Identification

code

EOTT ENERGY OPERATING LTD

Bakersfield, CA

EOTT ENERGY PARTNERSHIP LP

Houston, TX

EPSILON SXSTEMS SOLUTIONS INC

Albuquetque, NM

San Diego, CA

EQUILON PIPELINE CO

Freeport, TX

256880-0003-1

EQUINOX

Chino Valley, AZ

283350-0001-8

ERC INC

Huntsville, AL

29]290-0601-7

291290-0002-5

ERCO

Cambridge, MA

$292000-0001-0$

$266420-0001-3$

$248770-0003-8$

ERDA RAFT RIVER GEOTHERMAL

Almo, ID

ERDMAN \& ASSOC INC

Madison, WI

ERDOELBEVORRATUNGVERNBAND

Hamburg, Germany

288910-000t-9

ERECON CORP

McLean, VA

2664600-0001-6

ERI SERVICES INC

Btidgeport, CT

ERICKSON JOHN H Ogden, LA

280350-0001-2

BRH CTY INDUSTRLAL DEVEL AGCY

Buffalo, NY

ERIE MINING CO

Tonawanda, NY

ERIS ENTERPRISES INC

Columbia, MD

ERNST \& ERNST

Washington, DC

ERNST \& YOUNG LLP

Washington, DC

ERSKINE GEORGE S

Eureka, CA

ESCOR INC

Northfield, IL

ESL INC Sunnyvale, CA

ESMERALDA COUNTY OF

Goldfield, NV

ESTERLINE ANGUS INSTRUMENTS

Speedway, IN

ETC GROUP INC

Albuquercyue, NM

Ford City, CA

Golden, $\mathrm{CO}$

Lakewood, $C O$

McKinney, TX

Monterey, CA

Daksale, LA

Portland, $O R$

San Francisco, CA

Seaside, CA
266480-0001-5

026810-0002-5

266670-0001-7

$113100-0001-8$

$266570-000 \mathrm{~L}-4$

268010-0001-2

$266500-0001-9$

265600-0001-X

$292770-0001-5$

106760-0002-9

244960-0017-2

244960-0015-6

244960-0012-1

244960-0007-5

244960-0005-9

244960-0009-]

244960-0014-8

244960-0006-?

244960-0016-4

244960-0008-3 


\begin{tabular}{|c|c|c|c|}
\hline Awardee & $\begin{array}{l}\text { Identification } \\
\text { code }\end{array}$ & Awardes & $\begin{array}{l}\text { Identification } \\
\text { code }\end{array}$ \\
\hline Tacoma, WA & $244960-0010-5$ & \multicolumn{2}{|c|}{ F W BALLOU SENIOR HIGH SCHOOL } \\
\hline Three Rivers, TX & $244960-0013-X$ & Washington, DC & $266620-00001-9$ \\
\hline Vancouver, WA & $244960-0011-3$ & \multicolumn{2}{|l|}{ F W E STAPENHORST INC } \\
\hline ETS INC & & Pointe Claire, Cвnada & $266630-0001-2$ \\
\hline Longview, TX & $150940-10002-0$ & \multicolumn{2}{|c|}{ FACTORY MUTUAL ENG ASSOC } \\
\hline EUGENE WATER \& ELECTRIC B & OARD & Norwood, MA & $266640-0001-6$ \\
\hline Eugene, OR & $176640-000]-7$ & \multicolumn{2}{|c|}{ FACTORY MUTUAL RESEARCH CORP } \\
\hline EVALUATION RESEARCH CORP & & Norwood, MA & $027520-0001-4$ \\
\hline Falls Church, VA & $027110-0001-X$ & \multicolumn{2}{|l|}{ FAGO JR EDWARD $\mathbf{T}$} \\
\hline Vienna, VA & $027110-0002-8$ & \multirow{2}{*}{\multicolumn{2}{|c|}{$\begin{array}{l}\text { Kansas City, MO } \\
\text { FAILURE ANALYSIS ASSOC INC }\end{array}$}} \\
\hline EVALUATION TECHNOLOGIES I & NC & & \\
\hline Arlington, VA & $027120-0001-3$ & Oakland, CA & $182840-0003-2$ \\
\hline EVANSTON CITY OF & & \multicolumn{2}{|l|}{ FAIRCHILD INDUSTRIES INC } \\
\hline City Manager & & \multicolumn{2}{|l|}{ Fairchild Space \& Electronies } \\
\hline Evanston, IL & $266530-0101-6$ & \multirow{2}{*}{\multicolumn{2}{|c|}{$\begin{array}{l}\text { Germantown, MD } \\
\text { FALCON PRODUCTS INC }\end{array}$}} \\
\hline EVANSVILLE CLN CITIES ALLI & NCE & & \\
\hline Evansville, IN & $286130-0001-0$ & Sı Louds, MO & $288370-0001-4$ \\
\hline EVERETT CITY OF & & \multicolumn{2}{|l|}{ FALLS MANUFACTURING INC } \\
\hline Utilities Dept & & Chathan, NY & $284700-0001-2$ \\
\hline Evereth, WA & $266540-0101-X$ & \multicolumn{2}{|l|}{ FALMOUTH TOWN OF } \\
\hline $\begin{array}{l}\text { EVERGREEN SOLAR INC } \\
\text { Waltham, MA }\end{array}$ & $252700-0001-6$ & \multicolumn{2}{|l|}{ Pablic School System } \\
\hline EVERSYS CORP & & \multicolumn{2}{|c|}{ FAMOUS CONSTRUCTION CORP } \\
\hline Novato, $\mathrm{CA}$ & $287580-0001-1$ & Austin, TX & $248350-0002-1$ \\
\hline EVNA CORP & & \multicolumn{2}{|l|}{ FANTLE WLL } \\
\hline Athens, OH & $266550-0101-3$ & \multirow{2}{*}{\multicolumn{2}{|c|}{$\begin{array}{l}\text { La Crosse, WI } \\
\text { FA RALTSNTS INSTITUTE }\end{array}$}} \\
\hline BUR TECHNOLOGIES & & & \\
\hline $\begin{array}{l}\text { Carlsbađ, CA } \\
\text { EXECUTIVE COACHING }\end{array}$ & $286950-0001-X$ & Occidental, CA & $266820-0001-4$ \\
\hline Golden, CO & $280420-000]-4$ & \multirow{2}{*}{\multicolumn{2}{|c|}{$\begin{array}{l}\text { Fargo, ND } \\
\text { FARKAS \& MANELLI }\end{array}$}} \\
\hline EXECUTIVE MANAGEMENT SYS & TEMS & & \\
\hline Aslington, VA & $266560-0001-0$ & \multicolumn{2}{|l|}{ Washington, DC } \\
\hline EXECUTIVE OFF OF THE PRESI & DENT & \\
\hline Washington, DC & $027310-0001-5$ & Torrance, CA & $283170-9001-X$ \\
\hline Environ Quality Council on & & \multicolumn{2}{|c|}{ FARM/SOLAR ENERGY WORKS } \\
\hline $\begin{array}{l}\text { Washington, DC } \\
\text { EXETER ASSOC INC }\end{array}$ & $027310-0301.4$ & $\begin{array}{l}\text { Summertown, TN } \\
\text { FARTRCH INC }\end{array}$ & $027940-0001-2$ \\
\hline LCMD & & San Diego, CA & $285740,0001-9$ \\
\hline Silver Spring, MD & $106770-0202-5$ & FAYETTEVILLE HOUSING AUTHC & ORITY \\
\hline $\begin{array}{l}\text { EXOIL SERVICES } \\
\text { Golden, CO }\end{array}$ & $266590-00001-1$ & Fayelteville, AR & $266940-000 \mathrm{~L}-4$ \\
\hline EXPERIMENTAL ENERGY ENVI & & Bethesda, MD & $279680-0001-7$ \\
\hline Allanta, GA & $266600-000]-1$ & FEDERAL AVLATION ADMIN & \\
\hline EXTRANUCLEAR LABORATORI & S INC & Washington, DC & $286850-0001-7$ \\
\hline $\begin{array}{l}\text { RXISONG, PA } \\
\text { EXXON OFICE SYSTEMS CO }\end{array}$ & $0,1300-0,000-0$ & cial Space Transp & \\
\hline Rosslyn, VA & $027420-00054$ & Washington, DC & $286850-0101-3$ \\
\hline EXXON PIPELINE CO & & Bethesda, MD & $028100-0007-7$ \\
\hline Frepport, TX & $292010-0000]-4$ & Golden, $\mathrm{CO}$ & $028100-0006-9$ \\
\hline EYRE GUS SERVICE WNE & & Knox ville, TN & $028100-0008-5$ \\
\hline Wentg, MD & $200(4)+(0000]-8$ & Porland. OR & $028100-0005-0$ \\
\hline & & FEDERAL DRUG A & \\
\hline & & Rockville, MD & $205120-0001-8$ \\
\hline$F \& E$ & & FEDERAL ENERGY ADMIN & \\
\hline GES INC & {$[00990-000]-6$} & ZRGY ADMIN \& DO & $20000000001-1$ \\
\hline Blacksburg, VA & 284750-0001-0 & Washington, DC & $266950-0001-8$ \\
\hline
\end{tabular}


Awardee $\quad \begin{gathered}\text { Identlfication } \\ \text { code }\end{gathered}$

FEDERAL EXECUTIVE INSTITUTE

Charionesvilhe, VA 028130-0001-9

FEDERAL FACILITATORS GROUP LLC

Falls Church, VA 287490-0001-2

FEDERAL LIBRARY COMMITTEE

Washington, DC 192380-0001-8

FEDERAL LOCK \& SAFE INC

Arlinglon, VA

238520-0001-7

FEDERAL NETWORK SERVICES INC

Redmond, WA 289720-0001-9

FEDERAL OCCUPATIONAL HEALTH

Golden, $C O$

$284160-0001-8$

FEDERAL POWER COMM

Washington, DC 266970-0001-5

FEDERAL PREPAREDNESS AGCY

Washington, DC

$266980-000]-9$

FEDERAL. RES SOUTHERN

Venta, VA

FEDERAL SALES SERVICE INC

Alexandria, VA

$266990-0001-2$

FEDERAL SECURITY SYSTEMS INC

Falls Chusch, VA.

FEDERATION OF MATLS SOCIETIES

Washington, $D C$

FEES PAINTING SERVICE

Albucquerque, NM

FELLER DENNIS WERNER

FTedericksburg, TX

FELLOWS LEE

St John, VQ

$028200-0001-0$

C

FEMP COMMUNITY REUSE ORG Harrison, $\mathrm{OH}$

FENIX \& SCISSON INC

Las Vegas, NV

Tulsa, $\mathrm{OX}$

FERMIONICS CORP

Simi Valley, CA

FERN ENGINEERING CO

Bourne, MA

FERRELL Gas INC Topman, CA

FWELITY SYSTEMS INC

Gaithersburg, MD

FIELDER MARY $\mathrm{V}$

Gainesvilie, FL

FINLEY OLIVER

Lawrence, KS

FINNIGAN CORP

San Jose, CA

Isotope Ratio Products

Bremen, Germany

FIORE INDUSTRIES INC

Albuquerquic, NM

Los Alamos, NM

FIRST BAPTIST CHURCH Aberdeen, SD

FIRTH SCHOOL DISTRICT \#59

Firth, W

191940-0002-6

$2643000-0001-x$

$272160-0001-3$

$284580-0001-6$

028230-0004-6

$028230-0002-\mathrm{X}$

$283180-0001-3$

$267040-0001-1$

$157250-0001-\mathrm{X}$

$289500-0001-6$

$272730-0000-9$

275630-0001-1

$124750-0002-3$

$124750-0101-1$

287730-0002-7

$287730-0001-9$

$267070-0001-2$

264880-0001-2
Awardee

Identification

code

FISCHER DENIS

Oakton, VA

$288720-0001-7$

FISHERIES RESOURCE MANAGEMENT

Lapwai, ID

267080-0001-6

FITTERER ENGN ASSOC

Oakmont, PA

$267090-0001-X$

FLAMBOYANT ANYESTMENT CO

Roanoke, VA

$267100-0001-X$

FLEET LINE PRODUCTS

Springfield, TN

$285240-0001-5$

FLEETWOOD HOMES INC

Hobmer Homes Inc

Worthington, MN

$028710-0101-4$

FLIGHT SYSTEMS

Newport Beach, CA

$267110-0001-3$

FLORENCE CITY OF

Florence, AL

$267120-0001-7$

FLORENCE NIGHTINGALE CIRCLE I

St Petersburg. FL

267130-0001-0

FLORIOA A \& M UNIVERSITY

Physics Dept

Tallahassee, FL

$124790-0101-6$

FLORIDA CONSUMERS' FEDERATION
West Palm Beach, FL

FLORIDA SOLAR ENERGY CENTER

Cape Canaveral, FL

267200-0001-2

FLORIDA STATE HOSPITAL

Forida City, FL

FLORIDA STATE OF

Tallahassee, FL

Energy Office

Tallahassee, FL

Executive Oflicer - Governor

Tallahassee, $\mathrm{FL}$

FLORIDA STATE UNIVERSITY

Tallahassee, FL

Oceanography Dept of

Tallahassce, FL

FLORIDA TECH UNIVERSTTY

Florida Solar

Orlando, FL

FLORIDA UNIVERSITY OF

IFAS Sponsored Programs

Gainesville, FL

028920-0001-7

$009440-0003.7$

$028940-0001-4$

028940-090]-1

$028940-0801-5$

028950-0001-6

028950-0901-5

FLOW PRODUCTS INC Morgantown, WV

FLOW RESEARCH INC

Sas Rafael, CA

Princeton Comb

Princelon, N]

028960-0201-4

$028970-2502-6$

$267210-0001-6$

$267220-0001-X$

$267220-0102-4$

FLOYD AGRICULTURAL ENERGY COOP Copper Hill, VA

FLUID SYSTEMS

Los Angeles, CA

$276060-0001-8$

$267270-0001-8$

FM TECHNOLOGIES INC

Alexandria, VA

$174560-0003-4$

FMC CORP

Clinton, TN

$029+50-00042$ 


\section{Identification \\ Awardee code}

Landover, MD

029150-0007.7

Princeton, N3

Eng Systems Div

Oakland, CA

$029150-0206-1$

FMH

Albuquerque, NM

$287650-0,001-3$

FOLE GARY $L$

Astion, $W$

267810-010t-9

FONASH R L

Summerland Key, FL

FOOTHILL DE ANZA CMTY COLL

Los Altos Hills, CA

$267280-0001-]$

FOOTHILLS ENG CON

Golden, $\mathrm{CO}$

FORCE 3 INC

Crofion, MD

FORCEY STEVEN W

Idaho Falls, ID

FORI BACON $\&$ DAVIS UTAH INC

New York, NY

FORD MOTOR CO

Destorn, MJ

Detroit, MI

Kansas City, MO

Visteon Automotive Systems

Dearbors, MI

FORESIGHT SCIENCE \& TECHNOLOGY

Port Townsend, WA

FOREST FUELS INC

Keene. NH

FOREST HILLS SCHOOL DISTRICT

Sidman, PA

FORMCAST INC

Denver, $C O$

FORNEY ENGINEERING CO

Addison, TX

FORT LARAMIE ENERGY CONSERY

Fort Laramie, WY

FORT LEWIS COLLEGE

Sociology Dept

Durango, $\mathrm{CO}$

FORT LEWIS FOUNDATION

Durango, CO

$133500-0001-0$

INC

203300-(0001-2

290300-0001-5

279930-0001-7

029240-0002-5

$029260-0001-4$

029260-0003-0

029260-0005-7

029260-0401-X

165080-0002-0

267300-0001-5

029300-0002-3

283750-0001-9

267310-0001-9

113490-0001-9

FORT MORGAN SCHOOL DISTRICT

Region 3

Fort Morgan, $\mathrm{CO}$

267330-0101-2

FORT SANDERS DEVELOPMENT CORP Knoxville, TN

FORTE SYSTEMS INC

Alexandria, VA

Vienna, VA

FOSTER ASSOC INC

Decatur, IL

Washington, DC

FOSTER D SNELL INC

Florbam Park, NJ

FOSTER KENNETH K

Tucson, AZ

$254380-00042$

$254380-0003-4$

$141060-0002-4$

$141060-0001-6$

$271320-0001-2$

$271340-0001-X$
029150-0005-0

Atwardee

Identiflcation

code

FOSTER MILLER ASSOC RNC

Waltham, MA

FOSTER WHEELER CORP

Livingston, N]

ROSTER WHEELER ENERGIES

Lakeland, FL

FOSTER WHEELER ENV CORP

Oak Ridge, TN

FOSTER-GLOCESTER REG SCH DEPT

Glocester, $\mathrm{Rl}$

$145230-0001-8$

FOUR LEAF FARM

Durham, NC

FOWLKES ENGINEERING

Bozeman, MT

FOXBORO ANALYTICAL

Houston, TX

FRANK'S CLEANING SERVICE

Carterville, IL

Murphysbore, IL

FRANKLIN EXTENSION SERVICE

Greenfield, MA

$266910-0001-3$

$267350-0001-3$

$267360-0001-7$

267400-0001-8

$267400-0002-6$

FRED C HART ASSOC INC

New York, NY

$268180-0001-0$

FRED HUTCHINSON CANCER RES CTR

Seatue, WA

Public Health Seiences

Sealt:te, WA

$170340-0001-8$

$170340-0101-4$

FREIMAN PARAMETRIC SYSTEMS INC

Camden, NI

$030060-0002-1$

Cherry Hill, NJ

030060-0001-3

FREMONT COUNTY JONNT DIST \#215

St Anthony, ID

$267010-0001-0$

FREMONT COUNTY SCHOOL DIST \#6 Kinnear, WY

$267430-0001-9$

FRENCH TROY R

Bartiesvilie, OK

$291020-0001-6$

FRIDEN NEOPOST

IMD

Carot Stream, IL

$230250-0103-8$

FRIEDELL DR HYMER

Cleveland, $\mathrm{OH}$

FRIENIDS OF ANLMALS INC

Tinton Fäls, NJ

FRIENISS OF LAUGHING BROOK Hampden, MA

FRIENDS OF THE EARTH

San Francisco, CA

$267440-0001-2$

$267460-000]-X$

$267470-10001-3$

029470-0001-3

FRONT EDGE TECHNOLOGY INC

Baldwin Park, CA

$113470-0001-1$

FRONT RANGE SCIENTIFIC COMP Boulder, CO

FRUMERMAN ASSOC INC

Pittsburgh, PA

$283810-0001-7$

FRY ALBERT

Potomac, MD

FUEL \& BNERGY CONS INC

New York, NY

214940-0001-6

$090290-0001-X$

267480-000]-7

$2674901-0001-0$ 

Awardee
Identification
code

FUEL CELL TECHNOLOGIES INC

New Milford, CT

$291590-0001-5$

FUEL INJECTION DEV CORP

Bellmawr, NJ

FUJITSU BUSINESS COMM SYSTEMS

Foster City, CA

FUME FREE INC

Stuart, FL

FUNDINGSLAND LYNN

Fargo, ND

FUTURE TECHNOLOGY INC

Washington, $D C$

FUTURES GROUP INC

Glastonbury, CT

Training Group Inc

San Antonio, TX

FYLLING \& SCRAPING

Washburn, ND

030350-0001-8

$260060-0001-6$

$291100-0001-1$

$27266000001-7$

$267500-0001-0$

$030480-0001-1$

030480-0]04-2

$293250-0001-6$

G

G \& R SALES

Seatlle, WA

G \& W ELECTRIC SERVICE

Blue Island, IL

G E CAPTTAL SPACE

Tueson, AZ

G E HARRIS CONTROL SYSTEMS

Melbourne, FL

G E HARRYS ENERGY

Calgary, Catrada

G L CORNELL CO

Gaithersburg, MD

G S SANITATION SERVICE

Watertown, SD

GABRJELSON JAMES

Mirneapolis, MN

GAFFEY CLINE ASSOC

Dallas, TX

GAIA NORTHWEST INC

Bothell, WA

GALAXY INC

Washington, DC

GaLLaTIN

Bend, $O R$

GALLUP INC

Rackville, MD

GARAWAY LOCAL SCHOOLS

Sisgarciete, OH

GARBAGE INC

Have, MT

GARDEN STATE RACING ASSN

Niles, II

GARDEN VALLEY SCHOOL DIST \#T

Garden Valley, ID

GARDNER MATHLAS v

Hope, RI

$267510-0001-4$

$267650-0001-1$

$286830-0001-x$

286320-0001-2

292790-(x)01-2

$159470-0001-6$

$236380-0001-\mathrm{X}$

$267660-10001-5$

$265230-0001-x$

$267670-0001-9$

030640-0001-2

$291770-0001-3$

289780-0001-0

267680-0001-2

$291940-0001-8$

$030900-0002-4$

283520-0001-2

$267690-0001-6$

GARDY VICTOR R

Shelbume, VT
Awardec

Identification

coxte

GARRETT AIR RESEARCH MFG

Torrance, CA

$267710-0001=x$

GARRETT CORP

Los Alamos, NM

GARRETT ENERGY RES \& ENG

Claremont, CA

$030970-0003-8$

GARRETT MARK J

Anchorage, AK

GARST PERRY

Quinton, OK

GARTEN FOUNDATION

Salem, OR

GARTNER GROUP IDAHO

Meridian, ID

GARTNER GROUP INC

Falis Chusch, VA

GARZA CONSTRUCTION CO

Yaikima, WA

GAS ENGINEERS INSTITUTION OF

London, United Kingdom

GAS PRODUCING ENTERPRISES INC

Houston, TX

GAS RESEARCH INSTITUTE

Chicago, IL

Natl Gas Vehicle Business Unit

Chicago, II.

GAS TECHNOLOGY INSTITUTE OF

Chicago, IL

Des Plaines, IL

030980-0001-5

$267700-0001-6$

$276480-0001-6$

$2856800-0001-0$

264480-0001-t

$238450-0003-1$

$031050-0002-x$

Edacation Dept

Chicago, IL

Energy Conversion \& Storase

Chicago, IL

Engintering Research Dept

Chicago, IL

Des Plaines, II

Industral Energy Utilization

Chicago, IL

Process Research Dept

Chicago, IL

$040130-0001-6$

$267820-0001-6$

$031080-0001-2$

$03] 080-010]-9$

GASIFICATYON SERVTCE INC

West Terre Haute, iN

GATES ENGINEERING CO

Bœkicy, WV

GaTEWAY 2000

Harrișon, $\mathrm{OH}$

GATHMAN-MATOTAN

Albuquerque, NM

GC MICRO CORP

Elberton, GA

Golden, $\infty$

$040040-0001-7$

$040040-0002-5$

$0400400101-3$

$040040-0201-X$

040040-030]-6

040040-0302-4

$040040-0401-2$

040040-0601-5

$268960-0001-5$

$267830-0001-X$

$242360-0004-0$

$267840-0001-3$

$242560-0002-\mathrm{X}$

242560-0003-8

GE CAPTTAL IT SOLUTNS FED SYST

Golden, $\mathrm{CO}$

283940-0001-0

Norcross, GA

$283940-0002-9$

GE HARRIS ENERGY CONTROL SYST

Calgary, Canada

291580-0001-1

GEBCO INC

Albuquetque, NM

$265290-0001-i$ 
Awardee

Identification code

GEC ALSTHOM T \& D CO INC

Blauvelt. NY

Charkeroi, PA

Hawthorne, NY

Wateriown, SD

Cogenel Alsthom Division

Blauvelt, NY

New York, NY

Paris, France

Spring Valley, NY

Villeubanne, France

Mesisurements Division

Hawthorne, NY

GEISINGER MEDICAL CENTER

Plant Engineering Dept

Danville, PA

GELCO INFORMATION NETWORK

Reston, VA.

GELFAND MARGO

Germantown, MD

GEM TECHANOLOGY INC

Miami, FL

GEMTIRE CORP

Palo Alto, CA

GEMINI GROUP HOME

Rело, NV

GEN COMMUINICATIONS OF SF

San Francisco, CA

GENAISSANCE PHARMACEUTICALS

New Haven, CT

GENE ROULEAU \& ASSOC

Silver Spring, MD

GENERAL ATOMICS

San Diegu, CA

Convair

Berkeley, CA

GENERAL CABLE CORP

Unionn, NJ

179360-0012-7

179360-0013-5

179360-0008-9

179360-0007-0

$179360-0112-3$

179360-0110-7

179360-0105-0

179360-0111-5

179360-0109-3

$179360-0308-8$

031210-0101-9

284890-0001-8

$291450-0001-8$

$238440-0004-6$

$287130-0001-2$

$267850-0001.7$

$267860-0001-0$

258800-0001-X

$288660-001) 1-9$

$031270-0001-4$

$031270-0302-1$

037290-0001-1

GENERAL DYNAMICS ADV TECH SYS Greensboro, NC

GENERAL ELECTRIC CO

Niskayuna, NY

$292060-000 \mathrm{l}-2$

St Pclersburg, FL

031310-0062-7

031310-0053-8

$031310-0063-5$

Corp Reseerch \& Dev Dept

031310-0762-1

Niskayuna, $\mathrm{NY}$

GENERAL EXHWIT \& DISPLAYS

Chicago, II

2679]0-0001-5

GENERAL LAND OFFICE OF TEXAS

Austin, $\mathrm{TX}$

GENERAL MOTORS CORP

Chevrolet Motor Div

WarTer, MI

Trans Systerts

Warten, Ml

267920-0001-9

$031360-0202-4$

$031360-0502-3$

GENERAL SERVICES ADMIN

Falls Church, VA

San Diego, CA

$031400-0009-X$

$031400-0051-0$
Awardee

Washington, DC

Aulomotive Commodity Center

Washington, DC

Construction Mimst

Manchester, $\mathrm{NH}$

Fed InTo Systems Support

Huntsville, AL

GSA Training Center

Washington, DC

Northwest Artic Region

Aubum, WA

Professional Services

Bastrop, TX

Washington, DC

Pub Bldgs Service

Washington, DC

Technical Services Dir

Huntsville, AL

GENERAL TRCHNICAL SERVICE

Upper Darby, PA

GENESEE JOENT SCHOOL DIST \#2B\%

Genesee, ID

GENESIS LABORATORIRS INC

Batavia, IL

GENESIS PAINTING INC

Loveland, CO

GENESHS PRODUCTS

Fort Wayne, IN

GENEX TECHNOLOGIES INC

Kensington, MD

GENSLER WILLIAM G

Tucson, AZ

GEO HEAT CENTER

Klansath Falls, OR

GEO-CENTERS INC

Gainesville, FL

GEOCHEM RESEARCH INC

Houston. TX

GEOKINETICS INC

Concord, $\mathrm{CA}$

GEOKINETIOS OIL SHALE GP

Concord, CA

GEOMAT ENGINEERING INC

Santa Ana, C.A

GEOMECHANICS INTERNATIONAL

Palo Aito, CA

GEOMETRICS

Berkelcy, CA

GEOPHYSICAL \$ERVICES INC

Denver, CO

GEORGE CONSTRUCTION CO

Cumberland, MD

CEORGE WASHINGTON UNTVERSTY

Washingkon, DC

Engr \& Applied Science Dept

Washington, DC

Identifination codle

$031400-0022-7$

$031400-3222-6$

$031400-4349 \cdot X$

$031400-4212.4$

$031400-4422-4$

$031400.4752-5$

$031400-4550-6$

$031400-4522-0$

$031400-4622-7$

$031400-3512-8$

$267930-0001-2$

$283590-0001-8$

290020-0001-4

$290410-0001 \cdot 1$

$267940-0001-6$

$289210-0001-1$

$282320-0001-5$

$292900-0001-5$

$031550-0002-3$

$031570-0001-2$

$031580-0001-6$

$267950-0001-x$

$286220-0001-X$

$283760-0001-2$

267960-0001-3

$267970-0001-7$

$031620-0002-5$

$031640-000 \mathrm{H}-4$

$031640-0801-5$

GEORGIA ENVIRONMENTAL

Atlanta, GA 
Awardee

Identification code

GEORGLA SOLAR COALTITON A.llanta, GA

GEORGLA STATE OF

Atlanta, GA

Waynesburg, PA

Energy Resources Ofice or Atlanta, GA

Macon Youth Development Ctr

Attanta, GA

OER

Atlanta, GA

Public Service Commission Allants, GA

GEORGIA UNIVERSTTY OF

Edocn Res a Bev Assn of GA Unv AtJanta, GA

GEORGIA.PACIFIC CORP

Toledo, $\mathrm{OH}$

GEOSCIENCE LTD

Solana Beach, CA

GEOTHERMAL DEVELOP GROUP

Richland, WA

268050-0001-7

GEOTHERMAL DEVELOPMENT ASSOC Reno, NV

GEOTRANS

$283310 \cdot 0001-3$

Reston, VA

268060-0001-0

GERBER CONCRETE CONSTRUCTION Salt Lake City, UT

$03 \div 900-0002-6$

GERBER EARL

Decatur, IN

265680-0001-9

GERC SHELTERS

Shrevepon, LA

$292420-00001-9$

GERMANNA COMMUNITY COLLEGE

Locust Grove, VA

GERMANY FEDERAL REPUBLIC OF

Bederal Minister Far Research

West Getmany, Georgia

GERSHON MECKLER ASSOC Washington, DC

GETAHEAD CONSTRUCTION Rifie, $\infty O$

GETTY OIL CO

Western Explaration \& Prdp Bakersfield, CA

GIANT INDUSTRIES INC

Houston, TX

GIBBS * COX INC

Arlìngton, $\mathrm{VA}$

New York, NY

GIBBS \& HILL INC

New York, NY

GIBSON OIL \& REFINING CO

Tupman, CA

GIBSON PAUL W

Baltimore, MD

GILBERT ASSOC INC

Reading. PA
268080-0001-6

$268090-0101-8$

106950-0002-0

$279910-0001-X$

031980-0103-X

$268110-0001-5$

$268 \mathrm{~L} 20-000 \mathrm{~T}-9$

268120-0002-7

$032010-0001-9$

$11 \$ 630-000 \mathrm{~L}-3$

$276270-0001-7$

$268130-000 \mathrm{l}-2$
Awardee

Identification

code

GILBERT/COMMONWEALTH INC

Reading. PA

$032090-0002-6$

GILCREST MELISSA J

Baltimore, MD

$273490-0001 \cdot 4$

GILLI PAUL V

Graz, Australia

GILMAN CITY R-IV SCHOOL DIST

Gilman City, MO

$268140-0001-6$

GKSS FORSCHUNGSZENTRUM

Geesthacht, Germany

$2 \$ 2840-000 !-6$

GLADSTONE CITY OF

Gladstont, MI

$289880-0001-3$

GLASS SERVICE LTD

Vselin, Siovakia

$268190-000]-4$

$289560-0001-8$

GLASSTONE DR SAMUEL

Oak Ridge, TN

$268200-0001-4$

GLAZER GUILFORD

Torance, CA

$268600-0001-5$

GLENN KENNETH

Ligonier, PA

$043690-0001-7$

GLENNALLEN SCHOOL LIBRARY

Glennallen, AK

268220-0101-8

GLENNS FERRY JNT SCH DIST $\# 192$

Glenns Ferry, ID

$283570-0001-0$

GLOBAL ENVIRONMENTAL SOLUTJONS

Las Veges, NV

$288530-0001-5$

GLOBAL INTERNET NETWORK SVCS

Eincoin, NE

GLOBAL SOLAR ENERGY

Wheat Ridge, $C O$

$274260-0001-\mathrm{X}$

GLOBAL SYSTEMS TECHNOLOGY

Yardley, PA

$285520-0001-6$

GLOUCESTER COUNTY

Chosen Frecholders Board of Woodbury, NI

GMI ENGINEERING \& MGMT INST

Physics Dept

Flint, MI

$2901100001-3$

GMIR

Manassas, VA

GNB INDUSTRIAL BATTERY CO

Charlotte, NC

Grand Forks, ND

$268230-0101-1$

GNOSTICS CONCEPTS INC

Menlo Park, CA

GOAD RONALD R

Evansville, IN

GODFREY CHOCHOL \& FRANCIS

Colonia, YS is nol a valid Country 268260-000]-6 Code.

GOERZ J W

Lexington, $\mathrm{KY}$

GOLD JEFF \& BRUCE BOYD

Nevada City, CA

GOLDEN JOHN

Golden, $\mathrm{CO}$

GOLDEN WEST REFINING CO

Los Angeles, CA
$032390-0301-5$

$283050-0001-X$

$200790-0005-3$

$200790-0004-5$

268240-000]-9

268250-0001-2

$032440-0001-0$

$270580-0001-8$

$268270-0001-X$

$032560-0002-9$ 
Awardee $\begin{gathered}\text { Identification } \\ \text { code }\end{gathered}$

GONZAGA UNIVERSITY

Chemistry Department of

Spokane, WA

GONZALES CONSULTING SVCS INC

Golden, $\mathrm{CO}$

GOOCH CHARLES $W$

Chapmansboro, TN

GOOD-ALL ELECTRIC INC

Fort Collins, CO

GOODIN RONALD JAMES

Louisville, MS

GOODING JOINT SCHOOL DIST \#231

Gooding, ID

GOODSON \& ASSOC INC

Lakewood. $\mathrm{CO}$

GOODWILL COMMUNITY SERVICE

Phoenix, AZ

GOODWILL INDUSTRIES INC

Allanta, GA

GOODWIN CONSULTING CO

Sun Ctty, AZ

GORDIAN ASSOC INC

New York, NY

Washington, DC

GORDON GARY E

West Plains, MO

GORDON RESEARCH CONFERENCES

West Kingsion, RJ

GORHAM INTERNATIONAL INC

Gorham, ME

GORHAM SCHOOL DEPT

Gorham, ME

GORMAN CO

Englewood, $C O$

GOSS ENGINEERS INC

Denver, $C O$

GOULD INC

Gould Laboratories

Rolling Meadows, IL

Ite Imperial Corp

Chalfort, PA

GOURDINE SYSTEMS INC

Livingston, N]

GOVERNMENT INSTITUTE INC

Washington, DC

18590-0301-X

230340-0001-4

268280-0001-3

190650-0001-1

268290-0001-7

264850-0001-1

032680-0003-7

292870-0001-B

$2274900002-5$

268300-0001-7

$032710-0001-8$

032710-0002-6

267800)-0101-5

$032760-0007-5$

032780-0001-3

$101300-0001-9$

$275650-0001-4$

$284940-0001-2$

$032830-0205-3$

$032830-0306-8$

$268320-0001-4$

GOVERNMENT

$032860-0002-7$

Manassas, VA

Sunnyvale, $C A$

$193830-0001-5$

193830-0005-8

GOVERNMENT TECHNOLOGY SVCS INC Golden, $\mathrm{CO}$

184840-0007-9

GOVERNOR MIFFLIN SCHOOL DIST

Shillington, PA

097590-0001-6

GOVT SCIENTIFIC SOURCE INC

Vienna, VA

GP TECHNOLOGY CORP

Landover Hills, MD

GRAHAM \& SCHLAGETER INC

Chicago, IL.
Awardee

Identification

coile

GRAM INC

Golden, $\mathrm{CO}$

GRAM RESEARCH \& DEVELOPMENT

Charlorte, NC

268360-000\}-9

GRAMLING ENG CORP

Cheyy Chase, MD

GRAND JUNCTION CITY OF

Grand Junction, $\mathrm{CO}$

GRAND RAPIDS PUBLIC SCHOOLS

Grand Rapids, MI

$268370-900]-2$

GRAND TRAVERSE COUNTY

Board of Commissioners

Traverse City, MJ

GRANDE PETER C

Phoenix ville, PA

GRANGER INC

Sunnyvale, CA

$287550-0001-0$

$282850-000 \mathrm{H}-\mathrm{X}$

GRANGER JAMES A

Tallahassee, FL

GRANGEVILLE JOINT \$CH DIST 241

Grangevile, ID

GRANT PAUL $\boldsymbol{w}$

Davis, CA

GRANT THORNTON LLC Vienna, VA

268380-0101-2

$276500-0001-X$

$280430-0001-8$

$270400-0001-6$

279720-0001-8

$268390-0001-x$

GRAYS HARBOR COMM ACTION Aberdeen, WA

GREAT LAKES RESEARCH CORP

Elizabethion, TN

292030-0001-1

GREAT SOUTHW Estes Park, CO

GREATER CARIB ENER \& ENVIR FND

Key Biscayne, FL

CNCL

268400-(0001-X

GREATER LAWRENCE SANTTARY DIST

North Andover, MA

GREATER LON ISL CLEAN CITIES

Hauppauge, NY

$149340-0001-1$

GREATER PAR

Desiver, $\mathbf{C O}$

GREATER PORTLAND COUN OF GOV

Porltand, ME

GRECH LEONARD

Capistrano Beach, CA

GREEN IMPACT

Green Building Conference

Austin, TX

GREEN INTERNATIONAL INC Sewicklcy, PA

268420-0101-3

279440-0001-7

REEN RIVER

Owensboro, KY

GREEN SULPHUR DISTRICT VOL

Sandstone, WV

IND

$268430-0001-0$

279460-10001-4

$289660-0001-0$

GREENBRIER ASSOC

Atlanta, GA

R \& M Systems Dl.

Albuquerque, NM

GREENE CITY COMM ACTION CNCL Xenia, OH
$286570-0101-2$

$268440-000 \mathrm{~J}-4$

268450-000]-8

$268460-0001-1$

$268470-0001-5$

$268470-0102-X$

268480-000L-9 
Awardee

Identification code

GREENFIELD ATTAWAY TYLER

San Rafael, CA

GREENING AMERICA

Washington, $D C$

GREENLANDSFLY A/S

Nuuk, Greenland

GREENLEE \& WINNER

Boulder, $\mathrm{CO}$

GREMMINGER GREG $w$

Ste Genevieve, MO

GRENADA COMPUTER SERVICES INC

Norristown, PA

GRIGGS PRODUCTION

EEO

San Francisco, CA

280380-0101-X

GRINER INC

Waltham, MA

GRIPS JOINT POWERS AGENCY

Napa, CA

GROOMAN GRAPHICS

Laramie, WY

GROUP FOR RECYCLING

Pittsburgh, PA

GRUMMAN ECOSYSTEMS CORP Melville, NY

GS MICRO CORP

Novato, $\mathrm{CA}$

GS TECH INC

Oak Ridge, TN

GTE SYLVANLA INC

Towanda, PA

Electmonic System Group

Mountain View, $\mathrm{CA}$

$268520-00011-X$

268530-0001-3

268540-0001-7

$268550-0000]-0$

$268560-0001-4$

285080-0001-4

$243940-0002-5$

$268570-0001-6$

$268570-0102-2$

GTE WTRELESS PRODUCTS \& SERVS Atlanta, GA

290160-0001-1

GUAM UNIVERSITY OF

Agana, GU

GUANTERRA

Golden, $\mathrm{CO}$

GUELPH UNIVERSITY OF

Guelph, Canada

GUENTHNER JOHN L

Newark, DE

GUERDON INDUSTRIES INC

Louisville, KY

GULA PATRICIA

Mounl Airy, MD

GULF WESTERN INOUSTRIAL Swarthmore, NY

$165720-0002-8$

$287840-0001-5$

$291280-0001-3$

$270840-0001-1$

$268580.0001-1$

$276240-000]-6$

$268610-0001-9$

GUN SHOP

Vincentown, NJ

$292440-0001-6$

GUTHMILLER EARTHMOVING INC Jamesiown, ND

GUTHRIE COUNTY EXTENSION SERV Guthrie Center, IA
Awardee

Identification code

H

H \& H PROTECTIVE SERY CORP

Seallue, WA

$268640-0101-6$

H J DEGENKOLB \& ASSOC

San Francisco, CA

$268660-0001-7$

H2A INC

Boise, ID

$268670 \cdot 0001-0$

HADLEY \& BENNETT INC

Henniker, NH

$268680-0001-4$

HAEFELY TRENCH

Ontario, Canada

Scarbotough, Canada

$250890-0002-6$

250890-0001-8

HAGAN CO

Sioux City, IA

$293260-0001-X$

HAHINEMANN MEDICAL COLLEGE

Biological Chemistry Dept

Pliladiphia, PA

HAINES BOROUGH PUBLIC LIBRARY

Haines, AK

HAINES BOROUGH SCHOOL DIST Haines, AK

HAIR MICHAEL

Lafayetle, LA

HAL KIRN \& ASSOC Chevy Chase, MD

HALE M

Bone Cave, TN

HALE STEPHEN H

Cambridge, MA

HALL MARK SERVICES

Sactamento, CA

HaLl OF STATES

Washinglon, EC

HALLIBURTON NUS CORP Álbuquerque, NM

HALLIBURTON NUS ENV CORP Aiken, SC

Consolting Services Group

Gaithersburg, MD

HALLIBURTON SERVICES

Weston, WV

HAMBURG ROBERT A Onma, WV

HAMILTON ASSOC INC

Basel, Switzetland

Denver, $\mathrm{CO}$

268690-0101-4

276070-0001-1

$268710-0001-1$

$2737100-0001-\mathrm{X}$

$268720-0001-5$

$272720-0001-5$

$268730-0001-9$

$265270-0001-4$

$268740-0001-2$

$279610-0001-1$

218010-0001-8

$218010-0102-2$

268750-000]-6

$277460-000]-0$

$034820-0002-6$

034820-0003.4

HAMILTON COUNTY OF

Treasurer

Cincinnati, OH

HAMPTON'S HONEY FARM

Tetonia, ID

$10] 480-0503-9$

HANDICAP OPPOR \& PROG IN EMPLM Waterbury, VT

$268760 \cdot 0001 \cdot X$

HANDY \& WAJDA CONSULTING GROUP

Oakland, CA

268770-0101-X

HANDZLIK DDS WALTER A

West Beod, WJ

$268780-0001-7$ 
Awardee $\quad \begin{gathered}\text { Identification } \\ \text { cosde }\end{gathered}$

HANFORD ENVIRON HEALTH FDN

Richland, WA

HANOVER PETROLEUM CORP

Dallas, TX

035090-0001-3

HANSEN JOHN A

Brighton, MA

268800-0001-0

$2688] 10-0001-4$

HANSEN SCHOOL DISTRICT \#415

Hansen, ID

HANSEN TIM

Wichila, KS

HANSON DAVID M

White Lake, SD

HANSON JOHN

Jefferson, MD

HAQUE INTERNATIONAL South Portland, ME

HARAMBEE INC Okfahoma City, OK

$264910-0001 \cdot X$

$280700-0001.5$

$268820-0001-8$

270820-0001-4

$268830-0001-1$

HARBISON WALKER REFRAC Pirtsburgh, PA

HARBRIDGE HOUSE INC Boston, MA

268840-0001-5

035230-0003-3

HARD COATING INC

Evansville, IN

$289640-0001-3$

HARDER JOHN D Anahola, HJ

HARDNETT \& ASSOC Washington, DC

HARLEY ELLINTON \& P YEE ASSOC Southicld, MI

HARMON ROBERT A Latham, NY

HARMONY PROJECT Charleston, SC

HARMS ROGER Wichita, KS

HAROLD F SCOTT CONTRACTOR Bridgevitle. PA

HARPSTER CONSTRUCTION CO Grand Forks, ND

HARRIS CANADA INC Chicago, IL

HARRIS T Butlex, KY

$270780-0001-3$

2914201-0001-7

268850-0001-9

268860-0001-2

$284170-0001-1$

$277670 \cdot 0001 \cdot \mathrm{X}$

$268870-0001.6$

268890-0001-3

$254450-0002-8$

$035480-0002-9$

HARRIS THOMAS D

Auburn, AL

HARRISBURG CITY OF Harrisburg, PA

HARRY DIAMOND LABORATORY Hyaltsville, MD

HARSHAW CHEMICAL CO

Crystol \& Electronics Solon, OH

2689 ]0-010L-3

HART GRAHAME Friday Harbor, WA

HARVARD UNIVERSITY

Kennedy School of Gorerament Cambridge, MA

$268350-0001-5$

$035710-2402-8$
268170-0001-7

Awardee

Identification

code

HARVEST ENERGY TECHNOLOGY

Sunt Valley, CA

$251150-0002-4$

HARVEY SAND \& GRAVEL INC Devils Lake, ND

$295930-0001-7$

HARWOOD JAMES V Honolulu, $\mathrm{HL}$

$270510-0001-2$

HASAM REALTY CORP Hallandale, FL

$268920-0001-0$

HASTRAP HERMAN Kaneohe, HI

HATFIELD SCHOOL DEPARTMENT

Hatfield, MA

$268930-0001-4$

$287060-0001-0$

HAWAII ELECTRIC CO

Energy Services Dept Honolulu, HI

035880-010]-8

HAWAII STATE OF

Community Services Office of Honglulu, $\mathrm{HI}$

$035900-0901-2$

Energ Office Honolulu, HI

$035900-0301-4$

HAWKEYE CONSTRUCTION INC Rudyard, MT

HAWORTH INC Las Vegas, NV

$287280-0001-3$

HAWPE CARLETON $w$ Majuro, MH

$174640-0006-4$

HAY ASSOC

Golden, CO

HAYWORTH INC Albuquerque, NM

HAZEN SCHOOL DISTRICT Hazen, ND

HEADWATERS COMPOSTES Three Forks, MT

HEALTH \& HUMAN SVCS DEPT OF Dallas, TX

Food \& Drug Admin

Gaithersburg. MD

Indian Health Services

Billings, MT

National Institutes of Health

Bethesdo, MD

Durham, NC

Rockville, MD

Natl Inst Oecup Sfty \& Hith

Washington, $\mathrm{DC}$

Social Security Admin

Battimore, MD

$268980-0001-2$

$268990-0001-6$

285980-0001-9

$269000-000]-0$

$292410-0001-5$

036100-0018-X

$036100-\mid 121-1$

036100-1519-5

036100-0810-5

036100-0820-2

036100-0804-0

$036] 00-1405-9$

036100-0401-0

HEALTH EDUC \& WELFARE DEPT OF

Shiprock, NM

Washington, $D C$

269010-0003-0

$269010-0001-4$

West Hyattsvil]e, MD

269010-0002-2

Healtb Resonrces
Rockville, MD

HEALTH STRATEGIES INC Miannisburg, $\mathrm{OH}$

$269010-0104-5$

$293060-0001-4$

HEART FELT FITNESS

Carlsbad, NM

$290890-000]-1$ 
Awardee $\begin{gathered}\text { Identification } \\ \text { code }\end{gathered}$

HEART OF IL CLEAN CITIES CORP

Peoria, IL

$286660-0001-5$

HEAT TRANSFER RESEARCH INC

South Pasadena, CA

269020-0001-8

HEAVERS RICHARD $M$

Kingoton, RI

277320-0001-3

HEBERT ROBERT T

Saratoga, CA

HEBREW UNIVERSTTY

Jerusalem, Istael

$269030-000 \mathrm{l}-1$

$036200-0001-8$

HEC INC

Nahant, MA

Natick, MA

HEIMSATH CLORIS ASSOC

Houston, TX

HEIN MTCHAEL

Hawkeye, IA

HEINDESELMAN RUSS

La Grange, MO

HELIODYNE

Richmond, CA

HELIUM BREEDER ASSOC Newport Beach, CA

San Diego, CA

HEMKER PAUL W

New Paris, OH

HEMLOCK SEMICONDUCTOR CORP

Hemlock, MJ

HENDERSON ZACHARY

Roswell, GA

HENLEY CONSTRUCTION INC Harrison, AR

HENRY FORD HOSPITAL

Ann Astor, MI

HENRY JOSEPH

Fayetteville, AR

Winslow, AR

HENRY M JACKSON FOUNDATION

Rockvillc, MD

HERBERT SCHNEIDER CORP North Conway. NH

HERBERT WADE

Hartsburg, MO

HERBERT ZIEN

Milwaukee, WI

HERCULES INC

West Valley, UT

hERITAGE INSTITUTE

Caste Rock, $\mathrm{CO}$

Englewood, $\mathrm{CO}$

HERITAGE REPORTING CORP Washington, DC

HERITAGE TECHNOLOGIES

Castle Rock, $\mathrm{CO}$

HERMAN BROS LOGGING

Port Angeles, WA

HERMAN MLLER INC

Tulsa, $O K$

$289290-0001-0$

289290-0002-9

$269040-0001-5$

273710-0001-3

$277870-10001-5$

$269050-0001-9$

269060-0001-2

$269060-0002-0$

036360-0001-2

269070-0001-6

$269080-(0) 01-X$

273350-0001-7

$036500-0002-4$

269200-0001-6

269200-0002-4

260540-0002-0

$269100-0001-3$

269130-0001-4

$269+20-000]-0$

036540-0002-9

283830-0001-4

283830-0002-2

$179470-0001-8$

$283400-0001-2$

$269590-0001-7$

205800-0005-2
Awardee

Identification code

Washington, DC

$205800-0006-0$

C/O Mid South Interiors Inc

Elbertorn, GA

$205800-0104-0$

Henriksen/Buller

Zeeland, MI

$205800-6201-2$

HERRON AL
Cottonwood, ID

HERRON STEPHEN A

McCloud, $\mathrm{CA}$

HEWLETT-PACKARD CO

Rockville, MD

Flagstaff, $A Z$

HIGH POWER INC

Casa Grande, AZ

Casper, WY

Loveland, $\mathrm{CO}$

HIGH TECHNOLOGY ASSOC

Fronton, TX

HIGH VOLTAGE POWER CORP

Westborough, MA

HIGH WIND ASSOC INC

Milwauke, WI

HIGHLAND GEOGRAPHKC INC

Burlington, VT

HIGHLINE COLLEGE

Physical Sctences Dept

Des Moines, WA

$269140-0001-8$

$286000-0001-7$

$036640-0010-2$

$290400-000]-8$

$169520-0022-4$

169520-0020-8

$169520-0021-6$

$269150-0001-1$

$269160-0001-5$

$269770-0001-9$

$290240-0001-7$

HILGER HENRY

Fort Wayne, IN

HILL COUNTY YOUTH RANCH Ingran, TX

HILL INTERNATIONAL INC

Willingtoro, NJ

HLLCREST EDUCATIONAL CENTER

Lenox, MA

HIILLNBRAND $5 \mathrm{~J}$

Knoxville, TN

HIMMELMANN ROBERT

Oxford, WI

HISPANIC-AMERICAN MULTI SVCS

Indianapolis, $\mathbb{I N}$

HISTORY ASSOC INC

Gaithersbuts: MD

HITECH ENGINEERING CO

Germantown, MD

HITECH METALLURGICAL CO

San Diego, CA

HITEK INC

Sweet Homc, $O R$

HITTMAN ASSOC INC

Columbia, $\mathrm{MD}$

Columbia, SC

HODEL GENE

Roanoke, IL

HOFFMAN ED J

Laramie, WY
HIGH DESERT INVESTMENT CO
162800-00002-8

$277990-0(0) 1-5$

$283300-010]-6$

$269190-0001-6$

$269210-0001-\mathrm{X}$

$293020-0001-\mathrm{X}$

$269220-0001-3$

$269230-0001-7$

$037050-0001-2$

$133780-0001-5$

$284360-0001-3$

269240-0001-0

$037070-0001-\mathrm{X}$

$037070-0002-8$

$267870-0001-4$

269250-0000]-4 
Awardee

Irentification cade

HOFFMAN ELECTRIC INC

Pittsburgh, PA

HOLBROOK PUBLIC SCHOOLS

Holbrook, AZ

HOLLTNGSWORTH VOL FIRE DEPT

Alto, GA

HOLMAN'S OF NEVADA INC

Las Vegas, NV

HOLTSBERG FREDERICK W

Pierce, FL.

HOLY CHILD SCHOOL OF THE Rye, $\mathbf{N Y}$

HOME IMPROVEMENT CO INC

Yakima, WA

HOME PARK COMMUN IMPROV

Atlanta, GA

HOMEDALE JONT SCH DIST \$370

Homedale, $\mathrm{ID}$

HOMEDALE PUBLIC LIBRARY

Homedale, ID

HOMER PUBLIC LIBRARY

Homer, AK

HOMESTEAD ENERGY SYSTEMS

Orford, NH

HON CO

Chicago, IL

Muscatine, IA

HONEYWELL INC

Albuquerque, NM

Atlanta, GA

Berkeley, CA

Roseville, MN

Engineering Research

Minneapolís, MN

Home \& Building Control

Minneapolis, MN

Research Center

Blooningion, MN

Technology Strategy Center

Golden Valley, $\mathbf{M N}$

HOONAH SCHOOLS LIBRARY

Hoonah, AK

HOOPER BAY HIGH SCHOOL

Hopper Bay, AK

HOOPER INSTITUTE

Walpole, NH

HOPE COLLEGE

Natural Sciences

Holland, MI

HOPEWELL DESIGNS INC

Alpharet!a, CA

HOPI TRIBE

Mining \& Mineral Res Off of Kykotsmovi Village, AZ

HORANSON BRAD

Minneapolis, $M N$

HORIZON DATA CORP

Des Moines, IA
$269260-0001-8$

269270-0001-1

$269280-0001-5$

$287500-0001-2$

$269310-0001 \cdot 2$

$073570-0001-\mathrm{X}$

269320-0001-6

ASSOC

$269330-0001-X$

283670-0001-3

$269340-0001-3$

269350)-0001-7

269360-0001-0

139910-0004-0

139910-0003-2

$037700-0015-3$

037700-(0)14-5

037700-(x)13-7

037700-(k)12-9

$037700-0802-2$

$037700-1002-7$

$037700-0901-0$

$269370-0001-4$

$269380-0001-8$

269390-0001-1

269400-0101-8

264230-0001-X

291540-0101-3

269420-0001-9

$203240 \cdot 0004-9$
$037700-0411-6$
Awordete

Idenfification code

$203240-0003-0$

$203240-0005-7$

$203240-0006-5$

203240.0002 .2

Phoenix, AZ

HORIZONS COMPUTER INC

Washington, $D C$

269430-0001-2

HORNE ENGINEERING SERVICES INC

Bel Ajr, MD

2581330-0002-7

Fairfax, VA

258030-0003-5

HORSMAN DARRYL G

Mendotri Heights, MN

$269440-10001-6$

HORTON JACK

Del Rey, CA

HOT METAL MOLDING INC Arkadelphia, AR

HOUSANG \& URBAN DEV DEPT DF Washinglon, DC

HOUSING AUTHORITY OF ST LOUIS

Energy Dept of

St. Louis, MO

HOUSING SERVICES OF CHARLOTTE

Charlotte, NC

$269450-000 \mathrm{~J}-\mathrm{X}$

$284550-0001-5$

$038030-0001-7$

HOUSTON CITY OF

Planning Dept Eec Gov Bd

Houston, TX

HOUSTON UNIVERSITY OF

Houston, TX

HOWARD RELOCATION GROUP

Clarkston, WA

Fairfax, VA

Florence, SD

Los Angeles, CA

Mead, CO

Portland, OR

Redding, CA

Sacramento, CA

West Linn, OR

HOWARD UNIVERSITY

Chemical Enpdueering Dept

Washington, DC

HOWE NOLAN

Neosho, MO

HOWERTON WLFRED M

Escondido, CA

HUDSON INSTITUTE

Croton On Hudson, NY

New York, NY

HUFFMAN PAUL

Pemberville, $\mathrm{OH}$

HUGH COLE SCHOOL

Wamen, RI

HUGHES-CALIHAN

Las Vegas, NV

HUMAN DRMENSIONS INST

Ojai, CA

HUMAN ENVIRON INSTITUTE FOR

San Francisco, CA
$291120-0101-5$

269460-0001-3

286500-010:-7

$038080-0001-5$

$151320-0062-2$

151320-0054-1

151320-0055-X

151320-006]-4

151320-0056-8

$151320-0057-6$

151320-0058-4

151320-0059-2

$151320-0060-6$

038130-1501-7

269470-0001-7

$269480-0001-0$

$269490-0002-2$

269490-0001-4

$269500-0001-4$

$272540-000]-7$

291210-0001-8

$269510-0001-8$

269910-000]-9 

Awardee
Identification
code

HUMAN SCIENCES RESEARCH INC

McLean, VA

269520-000]-1

HUSKY OIL CO INC

New Orleans, LA

Santa Maria, CA

HY-TECH RESEARCH CORP

Radford, VA

$038630-0001-3$

038630-0002-1

288230-0001-7

HYACINTHETLCS CORP

Lake Charles, LA

HYDRAMECH TECHNOLOGES

Sumtier, WA

HYDROCARBON RECYCLERS

Tulsa, $O K$

HYDROCARBON RESEARCH INC

McLean, VA

Trenton, $\mathrm{N}$ J

HYOROCOIL NAN CO

Los Alamitos, CA

HYDROGEN BURNER TECH INC

Long Beach, CA

Fuel Cell Dept of

Long Beach, CA

HYDRONAUTICS INC

Launel, MD

HYGEIA RESEARCH ASSOC INC Commack, NY

269530-0001-5

284130-000]-7

269550-0001-2

038740-0006-0

038740-0007-9

$269560-0001-6$

284830-(0001-6

284B30-0101-2

038750-0001-3

292750-0001-8

HYPER-THERM HIGH-TEMP COMP INC Hurnington Beach, CA

HYPERION CATALYSIS INTL Cambridge, MA

HYPERNEX INC State College, PA

HYTEC INC

Los Alamos, NM

HYUNDAI CORP

Gardena, CA

258750-0001-5

$290200-0001-2$

$289120-0001-2$

$283790-0001-3$

$289710.0001-5$

\section{I}

1 SHEINBALM CO INC

Paşadena, CA

IBM GLOBAL Denver, $C O$

IBM NNC Hopewell Junction, NY

IBS SPARTAN

Savage, MD

ICF BUILDERS \& CONSULTANTS INC Nashville, TN

ICF INC

New York, NY

Washington, $D C$

ICF KAISER ENGINEERS INC

Oakland, CA

ICN BIOMEDICALS

Cleveland, $\mathrm{OH}$

IDAHO FALLS CITY OF

Mayor Office of

Idaho Falls, ID

269620-0001-4

289090-0001-5

269670-0001-2

158580-0002-9

2881 10-0001-7

269610-00002-9

$269610-0001-0$

190750-0002-2

$282780-0001-8$

038970-0601-4
Awardee

Identification

code

DAHO FALLS PUBLIC LIBRARY

Idaho Falls, ID

DAHO STATE OF

Energy Office of

Boise, ID

Euvinonmental Protection Aqcy

Boise, ID

Fish \& Game Dept

Boise, ID

Nationtai Guard

Boise, ID

Vocationad Education Div

ldaho Fails, ID

Water Resources Dept of

Boise, ID

IDAHO STATE UNIVERSITY

Id Center Snnall Accekerstors

Pocatello. ID

$269680-0001-6$

$039020-0601-3$

$039020-2401-1$

$039020-1701-5$

$039020-2501-8$

$039020-2607-3$

039020-0401-0

039030-0601-7

IDAHO UNIVERSITY OF

Accounting Services

Moscow, ID

Biological \& Agric Engrg

Moscow, ID

IDEAMATICS INC

Washington, $D C$

IEA EA TECHNOLOGY LTD

Capenhurst Chester, United Kingdom280370-0001-X

IEA PROJEKTLEITUNG ENERGIETORS Julich, Germany

IFE ENVIRON ANALYSIS LAB

Richmond, CA

269700-0001-X IHS

Englewood, CO

IKON OFFICE SOLUTIONS

Phoenix AZ

ILL BASIN COAL MI MP COV Indiznapolis, IN

$039040-2101-\mathrm{X}$

039040-2001-3

269690-0001-X

LLINOIS COAL GAS GROUP

Chicago, IL
ILLINOIS COMMUNTTY COLLEGE East Peoria, IL

ILINOIS INSTITUTE OF TECH

Clvil Engineering Dept

Chicago, It

ILLINOIS STATE OF

Capital Development Board

Springfield, IL

Commerce \& Cinty Affs Dept of

Springfield, IE

ILLINOLS TOOL WORKS Elgin, IL

ILLINOIS UNIVERSITY OF Chicago, IL

Electrical Engineering Dept Urbana, IL

I,O TECHNOLOGY INC

Surnyvale, CA
$269710-000 \mathrm{~L}-3$

$283060-0001-3$

$287590-0001-5$

$269720-000]-7$

269730-0001-0

$269750-0001-8$

039] 30-1401-2

1)39]40-2101-2

039140-1801-]

269740-0001-4

039170-0002-4

039]70-1103-4

269760-0001-1 
Awardee

IMAGE ASSOC Washington, DC

MAGE EXPRESS

Los Angeles, CA

IMDT INC Springfield, VA

IMMUSOL INC San Diego, CA

IMPERIAL WIRE \& CABLE New1own, PA

IMPRESSION 5 MUSEUM Lansing, MI

INACOMP Oakland, CA

INCELL CORP Shorewood, WI

INCO EUROPE LIMITED Birmingham, United Kingdon

INCO UNITED STATES INC Oak Ridge, TN

INDEPENDENCE CITY OF Independence, $M O$

INDIANA BIOLAB Palmyтa, IN

INDIANA COMM FOR EARTH DAY

Earth Day 80 Indiana Indianapolis, $\mathbb{N}$

INDIANA STATE OF

Commerce Dept of Henryvilk, IN Indianapolis, $\mathbf{N}$

Family \& Social Services Adm Indianapolis, IN

INDIANA UNIVERSITY

Chemical Sciences Bloomington, IN

Geological Survey Bloomington, IN

INDUSTRLAL BYOTEST LABS Northbrook, IL

INDUSTRLAL DESIGN MODELS Mount Clemens, MI

INDUSTRIAL ECOLOGY CENTER

National Defense Center

Picatinny Arsenal, NJ

INDUSTRIAL QUALITY INC

Gaithersburg, MD

INDUSTRLAL SCI \& TECH NETWORK York, PA

INDUSTRY CONSULTING Midlothian, VA

INFO ENGINEERING CONSORTIUM

Deale, $\mathrm{MD}$

INFORMATICS INC Golden, $C O$

Intormation Program Division Rockville, MD
Identification code

$269770 \cdot 0001-5$

$285670-0001-7$

$288070-0001-6$

$288040-0001.5$

$290440-0001-2$

$269780-0001-9$

$256550-0002-4$

$269790-0001-2$

269800-0001-2

$269810-0001-6$

039400-0002-7

$269840-000]-7$

$269850-0101-7$

039590-0102-9

039590-0101-0

039590-1201-2

$039620-0801-2$

$039620-0901-9$

$269860-900]-4$

$269870-0001-8$

$287360-0101-5$

$289750-000]-X$

$284560-0001.9$

269880-0001-1

$293050-0001-0$

039850-0003-4

039850-0102-2
Awardee

Identification code

\section{INFORMATION AGENCY US \\ Washington, DC \\ INFORMATKON BUILDERS \\ Arlington, VA \\ INFORMATION DESIGN ASSOC}

Sausalito, CA

INFORMATION DIMENSIONS INC

Golden, $\mathrm{CO}$

$283850-0001-1$

283440-0001-7

$285140-0001-2$

INFORMATION MGMT CONSULTNT INC

McLean, VA $236940-0001-1$

INFORMATION PLANNING ASSOC

Gaithersburg, MD

039870-0001-5

Rockville, MD

039870-0002-3

INFORMATION TELEVISLON NETWORK

Boca Ralon, FL

287950-0001-1

INFOSYS INTERNATIONAL Melville, NY

INFRARED INC

Reno, NY

292150-(0001-1

INGERSOLL RAND COMPR SYSTEMS

Tulsa, oK

INGRAM ANNE

Bethesda, MD

INMAC

Norwod, NJ

269890-0001-5

INNOVARE INC

Bath, PA

INNOVATECH INC

Dusham, NC

INNOVATIVE SYSTEMS INC

Kansas City, KS

t82450-0004-3

INSIGHT

Tempe, AZ

INSIGHT TECHNOLOGY

Londonderty, NH

$289840-0001-9$

252210-0001-6

$2860600-00001-9$

$281770-0001-2$

INSTITUTE FOR ENV MGMT INC

Pajo Alto, CA

288270-0(0)]-J

NSTRUMENT

Charlotle, NC

$288520-0001-1$

INSULATING SHADE LTD PTS

Guilford, CT

NNTEC SERVICES INC

Fort Collins, CO

INTEGRAL URBAN HOUSE

Berkeley, CA

INTEGRATED ENERGY SYSTEMS INC

Chapel Hill, NC

RP

292050-0001-9

269950-000]-3

$254430-0002-0$

$269970-0001-0$

INTEGRATED OFFICE SYSTEMS Gaithersburg, $M D$

NTEGRATED SERVICES CORP

Housion, TX

269980-00001-4

CORP

269990-0001-8

INTEGRATED SOFTWARE SYSTEMS CO Washington, DC

INTEGRATED VEG \& INSECT MGMT Vancouver, WA 287900-0001-3

INTEGRITY NETWORKING SYSTEMS Albuquerçue, NM

$289070-0001-8$ Tucson, AZ 
Awardee

Identifieation cole

INTELLIGENT FIBER OPTIC SYSTEM

Mountain View, CA

289010-0001-6

INTELLISENSE CORP

Wilmington, MA

$220860-0002-2$

INTER SYSTEMS INC

Washington, DC

$170840-0002 \cdot \mathrm{x}$

INTERDISCIPLINARY GRP FOR ECOL

Los Angeles, CA

$040240-10001-2$

Santa Monica, CA

INTERIOR DEPARTMENT OF

Golden, CO

Hemdon, VA

Fiș \& Willitife Service

Golden, $\mathrm{CO}$

Hejena, MT

Spokane, WA

Geologlcal Survey

Tacoma, WA

$040240-0002-0$

$040250-0030-6$

040250-0086-5

$040250-0780-0$

040250-07A7-H

040250-0779-7

Tutmers Falls, MA

Insular Afifairs Office of

Washingtod, DC

$040250-0830-0$

040250-08A9-K

040250-3232-5

Land Mangement Bureatu of Tulst, OK

Navajo Area Indian Health Sre

Window Rock, $A Z$

INTERMIETRCS INC

Greespel, MO

040250-34BO-B

040250-33A8-H

265580-0001-8

INTERNATIONAL \& DOMESTIC DEV

Paducah, KY

040300-0903-7

INIERNATIONAL ASSN ETC

Coral Gables, FL

$2700100001-9$

INTERNATIONAL BUSINESS SERVICE

Waşhington, DC

$270020-000 \mathrm{~L}-2$

INTERNATIONAL CONF ON RAD RES

Argonne, IL

$270150-0001-6$

INTERNATIONAL DATA CORP

Waltham, MA

$181700-0002-5$

INTERNATIONAL ENERGY AGENCY

Oxfordshire, United Kingdom 040420-0005-3

INTERNATIONAL ENGY \& ECON DEV

Boulder, $\mathrm{CD}$

270100-0001-8

INTERNATIONAL HARVESTER

Los Angeles, CA

San Diego, CA

$270030-0001-6$

$270030-0002.4$

INTERNATYONAL MANAGEMENT DEVEL Springfield, VA

$273340-0001-3$

INTERNATIONAL NICKEL CO

Suffem, NY

$270040-0001 \cdot X$

INTERNATIONAL PETRQLEUM SVC

Ei Dorado, KS

$270050-0001-3$

INTERNATIONAL POWER TECH

Sunnyvale, CA

$270060-0001-7$

INTERNATIONAL PRE-HEATER

Seattle, WA

$270070-0001-0$

INTERNATIONAL RADHATION PROT

Washinglon, DC 270080-0001-4

INTERNATIONAL RES \& TECH CORP

McLean, VA

040520-0002-1
Awardee

Identification

code

INTERNATIONAL RES INFO SYST

Fairfax, VA

040540-0001-0

INTERNATIONAL SCIENTIFIC INST

Santi Clara, CA

270090-0001-8

INTERNATTONAL SOC EXP HEMATEL

Bethesda, MD

$269960-000\}-7$

INTERNATIONAL SOC PLANT/MOL BI

Athens, GA

$124940-0003.3$

INTERNATIONAL SOLAR ENERGY SOC

Publictotions Depl

Killeen, TX

$040600-0201-1$

INTERNATL TRAINING SCHOOL FOR Brattleboro, VT

124070-0001-3

INTERNET DATACOMM INC

Lisle, IL

$270110-000 \mathrm{~L}-1$

INTERNET SYSTEMS INC

Germantown, MD

$204890-0001-0$

INTERSTATE ELECTRONICS CORP Anaheion, CA 270120-0001-5

INTERSTATE RENEWABLE ENERGY CO Albany, NY Lathan, NY $237530-0001-9$ $237530-0002-7$

INTERTECHNOLOGY/SOLAR CORP Warrenton. VA 040690-0001-1

INTERUNIV COM CNCL \& EDU COM Princeton, NJ

$270140-0001-2$

INTL AMERJCAN DEVELOPMENT

Washington, DC

285!30-0001-9

INTL BROTHERHOOD OF TEAMSTERS Mercury, NV $293220-0001-5$

INTL COUNCIL FOR LOCAL ENVIR

Berkeley, CA $280300-0001-4$

INTL FED OF INST FOR ADV STUDY

Toronto, Canada 283980-0001-5

INTL INST FOR INDIGENOUS RESOU Denver, $C O \quad 290870-0001-4$

INTL SCH OF ADVANCED SFUDIES Trieste, Ilaly 288620-0001-4

FNTL UTILITY STRUCTURES INC Scotisdale, AZ

$288750-0001.8$

IOGCC INTERSTATE OIL \& GAS Okbahoma Cily, OK

$284920-0001-5$

IOTECH CORP LTD

Otawa, Canade

$270160-0001-X$

IOWA CENTER FOR LOCAL RELLANCE

Des Moincs, IA

270170-0101-X

IOWA ENERGY POLICY COUNCIL Alnes, IA

04079)-0002-2

lOWA STATE OF

Human Rights Dept of

Des Moines, $1 \mathrm{~A}$

IOWA UNTVERITY OF

Mechanical Engineering Dept.

Jowa Cily, lA

Physics \& Astronomy Dept

lowa City, IA

IP FARMS INC

Houston, TX
040800-0501-6

$040820-0801-2$

$040820-0601-X$

$038800-000[-8$ 
Awardee

IRBY CONSTRUCTION CO

Jonesboro, AR

IRELAND JR WLLIAM

Georgetown, DE

IRI INTERNATIONAL CORP

Beaument, TX

IRVINE UC

Physics Dept

Irvine, CA

IRVING BURTON ASSOC INC

Elberton, GA

Oak Ridge, TN

ISC TELECOM INC

Englewood, $\mathrm{CO}$

ISOMETRICS INC

Reidsville, NC

ISSI INC

Silver Spring, MD

IT CORP

Washington, DC

Alaska Division

Las Vegas, NV

ITC CORP

Warrenton, VA

ITE IMPERIAL CORP

Greensburg, PA

Spring Housc, PA

ITEK OPTICAL SYSTEMS

Lexingıon, MA.

ITN ENERGY SYSTEMS INC

Wheat Ridge, $\mathrm{CO}$

ITT DEFENSE \& ELECTRONICS Roanoke, VA

IVANNA EUDORA KEAN HIGH SCHOOL

Charlote Amalie, VI

IVENTEK CORP

New Lenox, IL

\section{J}

I L INDUSTRIES INC

Glendive, MT

I \& L JANITORIAL

Baltimore, MD

J A JONES CONSTR CO

Argonise, IL

J BARRY SIMMONS \& ASSOC INC Baton Rouge, LA

J C SCHUMACHER CO

Oceanside, CA

J D G ASSOC INC

Sata Antonio, TX

J E NEWELL DECORATORS INC Washinglon, DC

J E NOTTI \& ASSOC

Fuilerton, CA

J E SIRRWE CO

Greenville, $\$ C$
270200-0001-0

288830-000]-3

2906600-0001-5

Identification

code

133900-0002-X

$282330-0001-9$

$150640-0002-2$

$270180-0101-3$

040920-0006-5

040920-0005-7

291670-000]-0

288150-0001-1

$287410-0001-3$

$145960-0005-0$

145960-0104-9

$270190-0001-0$

$269640-0001-1$

$269640-0002-\mathrm{X}$

269630-000]-8

$289040-10001-7$

284880-0001-4

$270210-0001-4$

$041150-0002-3$

$265360-0001-3$

278230-0001-6

288570-0001-X

$270220-0001-8$

$270230-0005-1$

$278730-0001-\mathrm{X}$
Awandee

Identification code

J F HOLMAN \& CO INC

Washinglon, DC

269300-000]-9

J K RESEARCH ASSOC INC

Artinglon, VA

$323440-0003-8$

J P MCGEHRIN \& ASSOC INC Upper Marlboro, MD

$257090-0001-9$

J R LUNDBERG \& ASSOC Poplar, WI

JACK FAUCETT ASSOC INC Chevy Chase, MD

$041280-0001-9$

JACKSON ROGER Oroville, WA

JACKSONVILLE ELEC AUTHORITY

Jacksonville, FL

JACKSONVILLE URBAN LRAGUE INC Jacksonville, FL 270340-0001-8

JACOBS ENERGY RESEARCH INC Audubon, $\mathbf{M N}$ $270350-0001-1$

JACOBS ENGINEERING GROUP INC

Albuquerque, NM

$041510-0002-X$

Houston, TX

041510-0004-6

Martinez, CA

041510-0003-8

Pasadena, CA

Richland, WA

$041510-0001-1$

041510-0005-4

Adranced Systems Dir

Pasadena, CA

Design Sci Div

Pasadena, CA

JAGCO DEVELOPMENT \& CONSTR

Las Vegas, NV

Mercury, NV

JALAR ASSOC

Wellesley, MA

JAMCO CONSTRUCTORS INC Diremt, $O K$

JAMES A LEWIS ENGNG

Dallas, TX

$041510-0101-8$

$041510-0201-4$

155590-0001-9

155590-0002-7

270360-0001-5

$253450-0003-4$

JAMIL SIMON PRODUCTIONS INC Cambridge, MA

$272280-0001-3$

JAPAN ATOMIC

270540-0001-3

Tokyo, Japan

$270550-0001-7$

JARRIEL J L

Aiken, SC

JASCO CONSTRUCTION SERVICES

Baker, MT

Mission, SD

Sidney, MT

JASON ASSOC CORP

Ashtabula, $\mathrm{OH}$

Dublin, $\mathrm{OH}$

Harrison, $\mathrm{OH}$

Mianisburg, $\mathrm{OH}$

JAY B HITCHING \& SONS

Downingeown, PA

JAZZ TECHNOLOGY USA LLC

Placentia, CA

JBF SCIENTIFIC CORP

Wilmington, MA
$270260-0001-2$

259820-0004-3

259820-0002-7

259820-0003-5

214980-0009-6

214980-0008-8

214980-0007-X

214980-0006-]

284410-000]-8

$286410-(0001-1$

041860-0002-6 
Awardee $\quad \begin{gathered}\text { Identification } \\ \text { code }\end{gathered}$

FDC INC

New Cumberland, WV

$28 \$ 810-0001-6$

JDG ASSOC INC

Boerne, TX

JEFFERSON COUNTY OF

Rigby, ID

JEFFERSON GREENHOUSES

Kearneysvilte, WV

JEFFERSON PARISH

Public Schools

Metaicie, LA

JEMICO INC

Columbia, MD

JENSEN ASSOC INC

Boston, MA

JEOL USA INC

Peabody, MA

JEROME UTE INC

Commack, NY

JERRY M BROWN CONSTRUCTION

Worland, WY

JETER SHELDON $M$

Atlanta, GA

JICKS BURUTA

Truk, FM

JIM BEABOUT CO

Littleton, $\mathrm{CO}$

JHMS BUILDING SERVICE

Cody, WY

JMJ ELECTRONICS

Oklahomia City, OK

JOE ROEBER ASSOC

London, Uniled Kingdom

JOHN C CAMPBELL FOLK SCHOOL

Brasstown. NC

JOHN FINCHYE FDN

Beloxi, WI

JOHN FITZGERALD KENNEDY LIB

Boston, MA

JOHN MUIR INSTITUTE INC

Los Alatros, NM

JOHNS HOPKINS UNIVERSITY

CBI Dept

Shendy Side, MD

Naval Se System

Silver Springs, MD

$232310-0001-7$

041950-0003-3

$270610-0001-5$

$270620-010]-5$

$270640+0001-6$

$042110-0002-0$

042120-0002-4

270650-0001-X

292430-0001-2

2706700001.7

270680-0001-0

$292890-0001-5$

$292590-0001-7$

$270700-0001-4$

62880.000t-9

$270730-0001-5$

$270800-0001-7$

270810-0001-0

$270860-000]-9$

JOHNS-MANVILLE PRODUCTS

R \& Center

Denver, $C O$

JOHNSON ALFRED

Oakland, CA

JOHNSON CITY MEMORIAL HOSP

Mouniain Ciry, TN

042440-2204-X

042440-2103-5

042450-0101-1

$128150-0002-4$

JOHNSON CONTROLS INC

San Diego, $\mathrm{CA}$

Walnul Creek, CA

Government Systems Group

San Diego, CA

$042520-0007-6$

$042520-0006-8$

$042520-0207-9$
Awardee

Identification

code

JOHNSON FLOWER \& GARDEN CENTER

Gaithersburg, MD

$164750-0002-7$

Gemantown, MD

JOHNSON FLOXD

Canton, $\mathrm{MO}$

$164750-0001-9$

JOHNSON G E

Knorville, TN

JOHNSON JAY

Excelsior, MN

JOHNSON LYNDON BAINES

Austin, TX

JOHNSON RON

Omaba, NE

$267260-0101-0$

$267520-0001.8$

$270930-0001-0$

$272650-0001-3$

JOHNSON RUSSELL G

Huron, SD

270940-0001-4

JOHNSON SHERRI

Victor, MT

$292630-0001-8$

JOHNSON W D

willoughby, $\mathrm{OH}$

JOINT CTR FOR POL \& ECON STUDI

Washington, $\mathrm{DC}$

JONT SCHOOL DISTRICT \#215

St Anthony, ID

JOINT TOWNSHIP DIST MEM HOSP

St Marys, OH

JOKEL A A AND GAGE STEPHEN

San Diego, CA

JONES E E

Mobile, AL

JONES EDWARD H

Richmond, VA

$278630-0001-7$

$270950-0001.8$

$289270-0001-3$

$267160-0001-1$

173060-0001-2

$270960-0001-1$

$270970-0001-5$

JONES ENGINEERING SALES INC

Cayce, SC

JONES RICHARD

Olyndon, MN

JONES WALTER F

Washougal, WA

JORDAN BROTHERS

Suffolk, VA

JORDAN LAWRENCE D

Rialto, CA

265950-0001-6

289580-0001-5

$277310-0001-X$

$281720-0001-4$

$270990-0001-2$

JOSLIN JEFF

Providence, RI

JOY EVEEYN

Silver Spring, MD

JOZEF STEFAN INSTITUTE

Jamova, Sierra leotre

JPR ASSOC INC

Stowe, VT

JRB ASSOC INC

McLean, VA

JRH MARKETING SERVICES New Yark, NY

$271000-0001-7$

$270590-0001-1$

$288120-0001-0$

$293300-0001-0$

$270270-000 \div-6$

$042900-0001-0$

$271050-0003.5$

JSA CO

Yuma, AZ
JUSTICE DEPARTMENT OF

Systems \& Ptanning Stafi

Washington, DC
$290700-0001-6$

$042960-1001-8$ 
Identification
code

$\mathbf{K}$

K \& A ENERGY CONSULTANTS INC Houston, TX

169670-0002-0

K N GAS GATHERING INC

K N Energy Inc

Brighton, $C O$

267800-0101-7

K R KOMAREK INC

Elk Grove Village, [L

KACHINA MOVING \& STORAGE

Albuquerque, NM

290950-0001-X

KAHN \& CO

Wethersfield, CT

KAHN AMERICAN HOFER INC

Wethersfield, CT

KALISPELL \$CHOOL DISTRICT \#S Pierte, SD

285300-0001-3

$271080-0001-6$

$271070-0001-2$

KAMAN SCIENCES CORP

Colorado Springs, $\mathrm{CO}$

043180-0002-8

KAMYR VALVES

Pittsburgh, PA

$157600-000]-2$

KANABLE JAMES H Mound City. SD

$279780-0001-\mathrm{X}$

KANKAKEE CON

$271090-0001-x$

Xankakee, IL

KANSAS CITY BOARD OF PUB UTIL Kansas City, KS

KANSAS STATE OF

Heelth Environment Dept of Topeka, KS

Weatherization Assistance Prog Topeka, KS

KANSAS UNIVERSITY OF Lawrence, KS

KAPPA SYSTEMS INC Arlington, VA

KARA FARM INC Whitewater, WI

KATTLESON MEMORIAL LIBRARY Sitka, AK

KAWECKI BERYLCO IND INC Reading, PA

KAYEX CORP Rochester, NY

KAYSINGER BASIN REG PLAN COMM Clinton, MO

KBB INC Washington, DC

$043290-1001-1$

043290-1101-8

043310-0001-X

043330-0002-5

$266880-0001-6$

$271140-0001.4$

$271150-0001-8$

271160-0001-1

$271170-0001-5$

KEEFE RIS \& 0 Chicago, IL

KEGOAYAH KOZGA LIBRARY Nome, AK

$269820-0001 \cdot x$

$277400-0001-9$

043480-0001-8

KELEHER DAN D

Sibley, MO

KELLER \& GANNON

San Francisco, CA

KELLOGG CO

Piscateway, NJ

$271210-0001-6$

$271220-0001-\mathrm{X}$
Awardee

Identification code

KELLOGG MW CO

Houston, TX

$271240-0001-7$

KELLY SERVICE

Albuguerque, NM

$271250-0001-0$

KEMPER MARI

Poulsbo, WA

$273000-0001-0$

KENAI PENINSULA COMMUNITY COL

Soldotna, AK

KENDRICK JOINT SCH DIST \#283

Kendrick, ID

$271260-0001-4$

KENNEGUNK LIGHT AND PWR DIST

Kennebunk, ME

KENNEDY VAN SUAN CORP

Danville, $\mathrm{PA}$

KENNETH E JOHNSON ENVIRON

Energy Center

Huntsville, AL

KENNY LAKE COMMUNITY LEAGUE

Copper Center, AK

$283600-0001-8$

KENNY LAKE COMM

(350-0001-3

Copper Center, AK

271270-0001-8

KENT CITY OF

Kent, WA

KENTUCKY COMMONWEALTH OF

Frankfort, KY

Lexington, KY

Lexington-Fayelte, $\mathrm{KY}$

043670-0001-X

Energy Cabinet

Frankfort, KY

Lexington, $K Y$

Energy Diviston of

Frankfort, KY

Energy Production \& Util Dept

Frankfort, KY

Lexington-Fayette, $\mathrm{KY}$

Nataral Res \& Env Prot Cab

Frankfort, $\mathrm{KY}$

Natural Resources \& Env

Frankfort, KY

Natural Resources Dept of

Frankfort, KY

RARY

$271360-0001-7$

Otl \& Gas

Lexington-Fayette, $\mathrm{KY}$

Social Insurance Dept for

Frankfort, KY

$271370-0001-0$

043820-0001-7

043B20-0002-5

043820-0003-3

$043820-0201-X$

$043820-0202-8$

043820-080k-8

043820-0501-9

$043820-0503-5$

043820-0301-6

$043820-060 \mathrm{~L}-5$

$043820-1001-2$

043820-0703-8

Special Projects Office of Frankfort. KY

$043820-0901-4$

KENTUCKY ENGY REGULATORY COMM Frankfort, KY

KENTUCKY RESEARCH FDN UNIV OF Lexington, KY

Agrononny Dept Lexington, $\mathrm{KY}$

$095890-0003-7$

Chemistry Dept

Lexington, $\mathrm{KY}$

$095890-0603-5$

KENTUCKY STATE OF

Geological Survey

Lexington, $\mathrm{KX}$
095890-0203-X

290970-0101-3 
Awardee

Identification

code

KENTUCKY UNIVERSITY OF

Extended Campus Program

Paducah, KY

Horticulture \& Land Arch

Lexington-Fayette, KY

KENTUCKY-WEST VA GAS CO

Prestonsburg, KY

KENZY CONSTRUCTION INC Gettysburg, SD

KEPLINGER \& ASSOC INC

Tulsâ, OK

KERN COLNTY REFINERY

Long Beach, $\mathrm{CA}$

KERN OIL \& REFINING CO

Long Beach, CA

KERNFORSCHUNGSANLAGE

Tipman, CA

KERR OLIVER

Miami, fL

KERSCHNER SWEENEY \& SWEENEY

Townsend, DE

KETCHIKAN PUBLIC LIBRARY

Ketchikan, AK

KETCHIKAN PUBLIC UTLITIES

Ketchikan, AK

043840-1503-8

043840-1002-8

$271390-0001-8$

286120-0001-7

$043890-0002-0$

$271400-0001-8$

$043930-0003-x$

$043940-0002-5$

$275640-0001-5$

$281450-0001-7$

$271410-0001-1$

KETRON INC

Phila Opr Eug \& Euv Sys

Wayne, PA

KETTLESON MEMORLAL LIBRARY Sitka, AK

KEV CORP

Washinglon, DC

KEVEX CORP

Foster City, CA

KEWANEE OIL CO

Tulsa, OK

KEYBOARD COMMUNICATIONS INC

Carle Place, NY

KEYSTONE CENTER

Boulder, $\mathrm{CO}$

KEYSTOHE SYMIPOSLA

Silverthorne, $C O$

$271420-0001-5$

$044020-0202-8$

$271440-0001-2$

279420-0001-X

$178110-0002-4$

$271460-0001-\mathrm{X}$

$044060-0002-X$

$110420-0003.4$

$288430-0001-2$

KIEFFER GORDON D

Wausau, Wl

KIEFFER SANITATTON

Rapid Cily, SD

KIGRE INC

Toledo, $\mathrm{OH}$

KLEARY SCOTT ASSOC Annapolis, MD

KDM TIMOTHY \& MARTHA

Roseville, $\mathrm{OH}$

KTMBERLY CLARK CORP

Neenah, W]

KINDSCHER KELLY

Saliza, KS

KINERGY RESEARCH \& DEV

Wake Forest, NC

$27[470-000]-3$

291640-0001-X

$271480-0001-7$

$271490-00001-0$

$284210-0001-2$

$271500-0001-0$

$271510-0000]-4$
Awardee

Identification code

KINETIC COATTNGS INC

Burlington, $\mathrm{MA}$

$271530-0001-1$

KINETIC SYSTEMS CORP

Lockport, lit

$271540-0001-5$

KINSMAN \& ASSOC

Corpus Christi, TX

$244980-0002-1$

Engineering

Richardson, TX

$210780-0101-4$

KIRA INC

Gertiantown, MD

KJRKPATRICK OFFICE OF

Brussels, Belgium

$208630-0005-3$

KIRSCHNER ASSOC INC

Washington, DC

KISS \& CATHCART

New York, NY

KLAMATH COUNTY YMCA

Klamath Falls, OR

KhaUS THOMAS J

Cooks, MI

KLEAN-RITE SERVICES INC

Oak Ridge, TN

KLEIN CONSTRUCTION CO

Westchester, Il

$044340-0001-2$

$271550-0001.9$

$285540-0001-3$

271570-0001-6

$271580-0001-X$

$221640-10002-1$

$2715900001-3$

KLEINMAN ROBERT E Golden, $\mathrm{CO}$

$2892500001-6$

KLEFTZ CHARLES

Union, $\mathrm{OH}$

$271600-0001-3$

KMC INC

Washington, DC

$16920000002-4$

KNEECAP NATURAL FOOD CO-OP

Providence, Rt

$271610-0001-7$

KNEELAND FAMILY PARTNERSHIP

Glenelg, MD

288190-0001-6

KNIGHT RIDDER INFORMATION INC

Arfington, VA

279410-0001-6

KNIGHT TOM

Tallulah, Falis, GA

KNIGHTSCENES INC

Washington, DC

$264500-0001-5$

KNOLL INC

East Greenville, PA

$264490-0001-5$

KNOM RADIO

Nome. AK

$285330-0001-4$

$271520 \cdot 0001.8$

KNOWLEDGE BASED SYSTEMS INC
College Station, TX

KNUDTSEN CHEVROLET CO Jantsville, WI

KODLAK ISLAND BOROUGH OF

Public Library

Kodiak, AK

KODLAK MENTAL HEALTH

Kodiak, AK

KOHRING GENE WILLLAM

Hamilton, $\mathrm{OH}$

KONHERS JEFFREY K

Wașington, DC
$271620-0001=0$

289300-0001-0

284710-0001-6

$230940-0101-7$

$271630-0001-4$

$267900-0001-1$

$270630-9001-2$ 


$$
\text { Awardee } \begin{gathered}
\text { Identification } \\
\text { code }
\end{gathered}
$$

KOOTENAI JOINT SCH DIST $\$ 274$

Harrison, ID

$114790-0001-9$

KOREA ATOMIC ENERCY RES INST

Yusung Taejon, Korea, Republic of 288670-0001-2

KORSGAARD \ENS

Princeton Junclion, NJ

KOTZEBUE CITY OF

Kotzebue, AK

KOTZEBUE ELECTRIC ASSN

Kotzebue, AK

KPMG PEAT MARWICK

Tulsa, OK

KRAMER CHIN \& MAY INC

Engimeering

Juneau, AK

KRELL INSTITUTE

Ames, LA

$290120-0001-7$

$285230-0001-1$

$285220-0001-8$

229030-(0002-6

271640-0101-4

$279130-0001-5$

KRILEY SOLAR DISTRIBUTORS

Hantisville, PA

KRIPIN TECHNOLOGIES INC

Rockille, MD

KRUEGER ENTERPRISES INC Cambridge, MA

KRUGEL LINDA

Portland, $O R$

KRUSE RICHARD W

Fort Calhoun, NE

KUEHL R I

Grants Pass, OR

KUHL CRAIG

SI Louis, MO

KUHN LOEB \& CO INC

New York, NY

KUJAWA PATRIClA S

Vienna, VA.

KUNA COMMUNITY LIBRARY Kuna, ID

KUSKOKWIM NATTVE ASSOC Anjak, AK

KVB EQUIPMENT CORP Tuștin, CA

KW TUNNELL CO INC Springfield, VA

KXL INC

La Due, MO

L

L'ECOLE POLYTECH DE MONTREAL

Metallurgical Eng Dept Montreal, Canada

LA CROSSE WISCONSIN WCA La Crosse, W1

LA MALOO

Pahor, HI

LA PLANTE DONALD A

White Mountains Regional High Whitefeld, NH

$271650-0001-1$

$284000-0001-3$

$271660-0001-5$

$272320-0001-4$

$277350-0001-4$

$271670-0001-9$

$289310-0001-4$

$271680-0001 \cdot 2$

$276250-0001 \cdot \mathrm{X}$

$271700-000 t-6$

$271710-000 t-x$

$271720-0001-3$

$291730-6001+9$

$166900-0002-8$

$271740-0101-7$

$271770-0001-1$

$271750-0001-4$

$264690-0101-7$
Awardee

Identification code

LA QUINTA MOTOR INNS

San Antonto, TX

$045080-0000]-0$

LA VETA SCHOOL DISTRICT

Region 2

La Veta, $\mathrm{CO}$

LABADIE JOHN R Seattle, WA

LABOR DEPARTMENT OF

Mine Safty \& Health Admin

Arlington, VA

Pittsburgh, PA

LABRENZ KIT

Dalton, WI

$045120-0101-8$

$271760-0001-8$

LAJET INC

Los Angeles, CA

LAKE \& PENINSULA \$CH DIST Nondalton, AK

LAKE AGISSIZ COUNCIL Moorhead, MN

045140-0201-1

045140-0208-9

$271560-0001-2$

$271790-0001-9$

$271820-0001-6$

LAKE MICHIGAN St Joseph, MI

LAKE REGION HOSPITAL Ottertail, $M N$

271800-0001-9

LAKE WASHINGTON SCHOOL DIST Juanita High School Kirkland. WA

045370-0101-1

LAKELAND CTTY OF

Lakeland, FL

$240250-1001-6$

LAKELAND COMMUNITY COELEGE Mattoon, IL

045420-0003-6

LAKESHORE BOARD OF GDUCATION Cleveland, WI

$271830-0001-X$

LAKESIDE ENGINEERING Mirror Lake, NH

$271840-0001-3$

LAKIN HOSPITAL Mason, WV

$271850-0001-7$

LAMAR COMMUNITY COLLEGE Lamar, $\mathrm{CO}$

$271860-0001-0$

LAMAR COUNTY MISSISSIPPI Purvis, MS

LAMAX CONSTRUCTION INC Basin, WY

$285470-0001-1$

LAMB ENGINEERING \& CONSTR CO Hardin, MT

LAMB R H AItoo, TN

LAMBROS LOIS Bethesda, MD

LANCIAULT JOSEPH A Fitchburg, MA
LAND DESIGN RESEARCH Columbia, MD

LAND INSTRUMENTS Nullytown, PA

LAND PETER Chicago, IL

LANDER CITY OF Lander, WY
$114910-0010-4$

$271870-0001-4$

$288340-0001-3$

$271880-000]-8$

$271890-0001-1$

$271910-0001-5$

$271920-0001-9$

271930-0001-2
$271900-0001-1$ 
Awardee

LANDIS \& STAEFA INC

Beltsville, MD

Hyattsville, MD

Las Vegas, NV

LANDMARK SYSTEMS Vienna, VA

LANE PLYWOOD CORP

Eugene, $O R$

LANGNER PARRY

London, United Kingdom

LANL FOUNDATION Santa Fe, NM

LANNING I MCCLURE

Slippery Rock, PA

LAPWAI SCHOOL DIST \#341

Lapwai, 10

LARRY BROWN \& ASSOC

Grants Pass, $O R$

LARRY RUSSELL ASSOC Houston, TX

LARSEN MANUFACTURING Huron, SD

LARSON \& TAYLOR Alexanktio, VA

Arlington, VA

LARUS CORP

San Jose, CA

LAS ANMAS SCHOOL DHST

Region\# 1

Las Animas, $\mathbf{C O}$

LAS COMUNIDADES

Vallecilos, NM

LAS VEGAS WEED \& SEED Las Vegas, NV

LASER VISION TECHNOLOGIES Colorado Springs, CO

LATIN AMERICA ENERGY ORGN Quito, Ecuador

LAURENS COMMUNITY Lajrens, la

LAURITE CORP San Diego CA

LAVELLE TOM Eugene, $O R$

LAW OFFICES STANTON KAY WATSON San Francisco, CA

LAWERTY DENISE Salmon, ID

LAWRENCE BERKELEY LABORATORY Berkeley, CA

LAWRENCE LIYERMORE NATL LAB Berkeley, CA

Livemore, $\mathrm{CA}$

272060-0001-0

Earth Sciences Division

Livermore, $\mathrm{CA}$

LAWRENCE REYNOLIS INC Richround, VA
$272060.0002-9$

272060-0102-5

Identification

code

$284640-0003-0$

284640-0001-4

$284640-0002-2$

$125000-0003-6$

$272670-0001=0$

271950-0001-X

$289060-0001-4$

271730-0001-7

$266830-0001-0$

271970-0001-7

$271980-0001-0$

$287290-0001-7$

045900-0003-2

$045900-0001-6$

$290420 \cdot 0001-5$

(45920-0101-X

$289140-0001-x$

$265030-0001-4$

279570.0001 .0

$280310-000+-8$

$272020-0001-6$

266680-0001-0

$272030-0001-X$

$286790-0001-9$

$272040-0001-3$

$272050-0001.7$

$272070-0001 \cdot 4$
Awardee

Identiffcation

code

LEADS CORP

Arlington, VA

LEAGUE FOR INNOV IN CMTY COLL

Los Angeles, CA

LEAGUE OF CITIES

Mayors Conf of

Washington, DC

$272090-0101-8$

LEAN ERIC G Chappaqua, NY

27210000001-1

LEAR MOTORS CORP Reno, NV

Sparks. NV

LEBANON TOWNSHIP ENV CO

Gien Gardner, NJ

LEBRUN JAY

Kent, WA

LECLERE GEORGE D

Montrose, $\mathrm{CO}$

LEE ALBERTA

Leeds, UT

LEE CASH

Atlanta, GA

2721 10-0001-5

$272110-0002-3$

$276260-0001-3$

$272120-0001-9$

$267980-0001-0$

264470-0001-8

$272130-0001-2$

LEE KRAMER ASSOC

Washington, DC

LEE LORDN D

Leeds, UT

LEE WAN \& ASSOC INC

Decatur, GA

Golden, $C O$

LEFEVER BLDG SYSTEMS INC

Craig, $\mathrm{CO}$

Montrose, $\infty$

LEGIN GROUP INC

Gaithersburg, MD

LEGIS 50

Denver, $C O$

LEGISLATIVE IMPRVMT CNTR FOR Denver, $\mathrm{CO}$

LEGORE J \& WILLIS T

Searsmont, ME

LEHIGH ROCAPPI

Pennsauken, NJ

LEMNITZER WILLIAM L Arlington, VA

LEROY EUGENE $P$ Merizo, GQ

LEROY PERCY STATE PARK Hobliston, MS

LESSER STAN

Chicora, PA

LETCO INC

Springfield, MO

LETOURNEAU UNIVERSITY

Engineeriag Dept

Longview. TX

$291300-0001-7$

046340-0001-6

$194870-0003-8$

$1948700004-6$

285280-0002-3

288280-000]-5

288930-0001-6

$272180-0001-0$

$272190-0001 \cdot 4$

$272200-0001-4$

272210-0001-8

$282350-0001-6$

$2665100001-2$

$266520-0001-6$

$272260-0001-6$

$272270-0001 \cdot \mathrm{X}$

$283290-0101-6$

LEVI RAY * SHOUP INC

Springfield, IL 
Awardee $\begin{gathered}\text { Identification } \\ \text { code }\end{gathered}$

LEWAN \& ASSOC

Golden, co

LEWIS \& CLARK STATE COLLEGE

Lewiston, ID

$286890-0001-1$

LEWIS PRITCHARD C

Crosswille. TN

$272290-0001-7$

LEWISTON INDEPENDENT SD \#340

Lewiston, ID

276980-0001-X

LEXIS NEXIS

Washington, DC

LIBRARY SYSTEMS \& SERVICES Germantown, MD

LIEBERT BROS ELECTRIC CO Coffeyville, KS

$283650-0000]-6$

$250100-0001-6$

$046890-0001-8$

LIFE ENHANCEMENT TECH INC Redwood City, CA

LIGHTING TECHNOEOGY CORP Burlingame, CA

$272300-0001-7$

$289590-6001-9$

LLLEMO KENNETH \& SCOTT HOLCOM Ames, iA

$271300-0001-5$

LILLICK MCHOSE \& CHARLES

San Francisco, CA

$120630-6001-8$

LINCOLN HOUSING AUTHORJTY

Energy and Kinetics Div of Lincoln, NE

LINCOLN TECHNICAL SERVICES Sudbury, MA

047070-0101-7

LINDSAY CADILAC CO

Alcxandria, VA

LINEAR PROGRAMMING I

Silver Spring, MD

LINEBARGER DICK

Fellon, CA

LINFIELD RESEARCH INST McMiznville, OR

LINN-RENTON COMM COLLEGE Albany, $O R$

$285630-0001-2$

$289570-0001-1$

$272330-0001-8$

$272340-000]-1$

$2723500001-5$

LIONEL HENĐERSON * CO Elk Grove, CA

LISENBY WILLIAM A Chesterfield, SC

LIST OLDS-CADILLAC Bartlesville, OK

LITTMAN STEVE Quincy, FL

LITYON PRC Reston, VA

LIVERMORE USD Liventore, CA

LIVING SYSTEMS Winters, CA

LLOYD WALLACE $R$ Sandpoint, ID

LOCAL SERVICES DEPT OF Comm S Diviston of Little Rack, AR

$272360-0001-9$

$290900-0001-1$

$272370-0001-2$

$272380-0001 \cdot 6$

$279920-0001-3$

$293110-0001-9$

$272390-0001 \cdot \mathrm{X}$

$272400-0001 \cdot x$

$277120-0005-8$

LOCKHART ELEMENTARY SCHOOL Charlote Amalie, VI
Awardee

Identification code

LOCKHEED AHCRAFT CORP

Burbank, CA

Carlsbad, CA

$272440-0001-4$

Sunnyvale, CA

LOCKHEED MARTTN ASTRONAUTICS

Degver, $\mathrm{CO}$

LOCKMAN \& ASSOC

Monlerey, CA.

$272440-0002-2$

$272440-0003-0$

287710-000:-1

LOCKWOOD GREENE TECHNOLOGIES

Miamisburg, OH

LOFTNESS MARVIN

Olympia, WA

LOGICON EAGLE TECHNOLOGY Arlington, VA

LOGICON INC

Tortance, CA

LOGISTICS APPLICATIONS INC

Washinglon, DC

LOGISTICS MANAGEMENT INST Cincinnati, $\mathrm{OH}$

LOGIUDICE THOMAS

New York, NY

LONDE-PARKER-MICHELS Clayton, MO

LONNECKER ADRIAN \& BRIDGENS $S$ Kansas City, MO

LOPEZ CONSTRUCMON CO

Bruceton, PA

LOPEZ ISLAND GRANGE 1060 Lopez, WA

LOPEZ JUAN M

Bayamon, $R Q$

LOPEZ QUARRIES

Everett, WA

LOS ALAMOS COUNTY OF

Los Alamos, NM

LOS ALAMOS NATIONAL LABORATORY Albuquerque, $\mathrm{NM}$

LOS ALAMOS PUBLIC SCHOOLS Los Alamós, NM

$273160 \cdot 0001-5$

$281110-0001-4$

208610-0002-1

$185520-0002-4$

$047590 \cdot 0003-8$

$272460-0001-1$

047610-0002-3

$272470-0001-5$

$134340-0002-X$

$272480-0001-9$

$272490-0001-2$

$272500-0001-2$

047830-000]-8

$291050-0001-7$

LOS ALAMOS TE 286670-000]-9

Idaho Ffalls, ID

LOS ANGELES CTTY OF

Euvironmentsl Aftairs Dept Los Angeles, CA

$047850-0201-8$

LOS GATOS RESEARCH Mountain View, CA

LOS RIOS COLLEGE Sactamento, CA

LOST RIVER COMMUNITY LIBRARY Aroo, ID

LOST RIVER RANGER DISTRICT Mackay, ID

LOTEC INC

Salt Lake City, UT

LOTUS DEVELOPMENT CORP

Westfield, MA
283190-0001-7

$272520-0001-X$

$272530-0001-3$

$283390-0001-2$

34350-0003-1

279580-0001-4

291460-000L-I 
Awardee

Identification code

LOUIS HARRIS \& ASSOC

New York, NY

$272560.0001-4$

LOUISLANA STATE OF

Social Service Dept of

Baton Rouge, LA

Wildilfe \& Fisheries Dept

Hackberry, LA

LOUISIANA STATE IDNIVERSITY

Enpironinentiol Stury Inst For

Balon Rouge, LA

LOVELACE BIOMED \& ENV RES INST

Albuquerque, NM

LOVELACE RESPIRATORY RES INST

Albuquerq̨ue, NM

LOW COST ENERGY CO

Greenville, SC

LOWE KING PRECE \& MARK

Arlingtor, VA

LOWELL UNIVERSITY OF

Engipeering College of Lowell, MA

Nłclear Eagineering Dept Loweli, MA

LOWER SAXONY STATE OF THE

Ministry Science \& Culture

Hannover, Gcrmany

LOYOLA UNIVERSITY

VA Hespital

Maywood, L

LSR REFINISHING

Clinton, MD

LTC AMIERICAS INC

Stetling, VA

LUCENT TECHNOLOGIES

Silver Spring, MD

Oxte] Messaghng Division

Fairfax, VA

LUDLUM MEASUREMENTS INC

Swoctwater, TX

LUDWTG WOLF

Crystal Lake, $\mathbb{L}$

LUGARDO LUIS M

San Juar, PR

LULEJLAN \& ASSOC Falls Church, VA

LUMMUS CO

Bruceton, PA

Chicago, IL

LUNAIRE LTD

Parsippany, NJ

LUNDBERG SURVEY INC

North Holfywood, CA

LUNDE MARTTN RONALD

Minneapolis, MN

LUTHER COLLEGE

Decorah, IA

LUTHERAN MEDICAL CENTER New York, NY
047960-0601-X

$047960-0403.3$

047970-1601-9

048040-0001-]

288630-000I-8

$290030-0001-8$

$2725700001-8$

$048080-0401-1$

048080-0501-8

288400-0101-8

$166270-0102-0$

289960-(x)01-9

$289950-0001-5$

$260670-0002-4$

$260670 \cdot 0103-9$

285760-0001-6

$282570-0001.9$

$272580-0001-1$

$272600-0001-5$

$048250-0003-7$

048250-0004-5

$272610-0001-9$

$272620-0001-2$

$271330-0001-6$

$102380-0004-4$
290430-0001-9
Awardee

Identification code

LUTHERAN WELFARE SERVTCE

St Charles, IL

$272630-0001-6$

LUX CONCRETE \& CONSTRUCMON 0

Jamestown, ND

290170-0001-5

LYMAN SCHOOL DIST 42-1

Presho, SD

$272640-0001-X$

LZP INC

Denter. $\mathrm{CO}$

$290390-0001-8$

$\mathbf{M}$

M D WOOD INC

Palo Alo, CA

272710-000L-1

$M$ S CASPE CO

Burlingame, CA

$140010-0002-4$

M S GINN \& CO

Hyattsville, MD

048590-0006-4

M-C POWER CORP

Burr Ridge, $\mathbf{L}$

MAC CONSTRUCTION CO INC Faith, SD

MAC DRILLING INC

Livonia, LA

MAC JAG TECH

Las Vegas, NV

MAC TECHNICAL SERVICES CO

Germantown, MD

MACGREGOR WILLIAM A

Geneseo, NY

MACK TRUCK INC

Allentown, PA

MACK TRUCK SALES OF TULSA INC Tulsa, OK

MACLEAN POWER SYSTEMS Franklin Park, IL

MACON CALYIN E Cataula, GA

MACON DIST EMP ASSN

Macon, GA

$202790-00001-4$

$292620-00001-4$

272740-0005-2

$283890-0001-6$

$158670-0004-4$

$272750-000 \mathrm{~L}-6$

272760-0001-X

$287440-0001-4$

$291570-0001-8$

$272770-0001-3$

MACRO METALICS DIV OF ELCON

San Jose, CA

MACTEC IDAHO DIVISION Idaho Fails, ID

MACTEC INC

Germantown, MD

Golden, $\mathrm{CO}$

Grand Iunction, CO

West Valley, NY

MAD DOG DESIGN \& CONST INC

Tallahasset, FL

MADANSKY DR CHARLES

BarTe, MA

MADGE NETWORKS INC

Eatontown, NJ

Golden, $C$

MADISON INC OF GEORGLA

Conyers, GA

MADISON RESEARCH CORP

Oak Ridge, TN
$272780-0001-7$

$283950-0001-4$

$283080-0001-0$

$283080-0002-9$

283080-0003.7

$283080-0004-5$

283080-0005-3

272790-0001-0

$276080-0001-5$

$258980-0002-X$

258980-0001-1

$272800-0001-0$

$252100-0002-8$ 


$$
\begin{aligned}
& \text { Identification } \\
& \text { code }
\end{aligned}
$$

MADISON SCHOOL DISTRICT \#321

Rexburg, ID

$264890-0001-6$

MAECO INC

Cape Girardeau, MO

$272810-000 \mathrm{H}-4$

MAERZ MICHAEL

Keokuk, IA

$273750-0001-8$

MAGIC VALLEY LIBRARY SYSTEM

Twin Falls, ID

$272620-000]-8$

MAGMA GULF CO

Baton Rouge, LA

MAGNETIC CORP OF AMERICA

Wailtham, MA

$272830-0001-1$

$272840-0001-5$

MAGRUDER JAMES $K$

Las Vegas, NV

289280-0001-7

MaHaLla SHaLOM

Phoetix, AZ

$272850-0001-9$

MAIL ADVERTISING Abilene, TX

$272860-0001-2$

MAIL AMERICA

Bryans Road, MD

MAINE AUDUBON SOCIETY

Falmouth, ME

MAINE HYDRO-ELEC DEVELOP CORP

Belfast, ME

MAINE MASTER ELEC PROD CO

Winterport, $\mathrm{ME}$

MAINE SCHOOL ADMIN

Frenchville, ME

MAINE UNIVERSITY OF

Orono, ME

MAjOR WLLIAM L

Glennallen, AK

MALHEUR NATIONAL FOREST

John Day, OR

MAN \& SCIENCE INSTITUTE OF

Rensselaerville, NY

MANAGEMENT ANALYSIS CO

San Diego, CA

MANAGEMENT COMMISSION Jackson, MS

MANAGEMENT SCIENCE SYST

Alexardsia, VA

Falls Church, VA

$272870-0001-6$

$272880-0001 \cdot X$

$272890-0001-3$

$272900-0001-3$

$049050-0015-7$

$049070-0002-2$

$282340-0001-2$

$272950-0001=1$

040110-0001-9

$272960-0001-5$

$282960-0001-6$

$272970-0001-9$

$272970-0002-7$

MANAGEMENT SYSTEMS DESIGNER

Vienna, VA

287100-000t-t

MANAGEMENT SYSTEMS INTL INC

Washington, DC

MANAZANITA BAND OF MISSION

Boulevard Gardens, CA

292350-0001-7

MANCOCK COMPREHENSIVE D Stanford, CA

$255040-0001-7$

272980-0001-2

MANHATTAN CMTY COLL BOROUGH OF New York, NY

MANHATTAN UNIVERSITY FOR

Manhattan, KS

224470-0001-4

MANHATTAN USD

Manhattan, KS

272940-0001-8

$272930-0001-4$
Awardee

Ideutification

code

MANSOUR SAMIR E

Upland, CA

$249300-0001-7$

MANUFACTURING \& TECH CONVERSN Battimore, MD

$174970-0003-9$

MAPEL J R

Lake City, IA

$270280-0001-X$

MARADA CORP

Fairmont, WV

$248710-0002-8$

MARCHETTI GEORGE A

Western Springs, IL

$285010-0001-9$

MARELCO INC

Alexandria, VA

$272990-0001-6$

MARIDAN INC

Washington, DC

MARTRMONT BOARD OF EDUCATION

Mariemont, $\mathrm{OH}$

MARIN SOLAR VILLAGE CORP

Inverness, CA

MARION COUNTY OF

Housing Anthority

Salem, OR

MARION SCHOOL DISTRICT Marion. IA

273030-0001-1

MARKET FACTS INC

Chicago, IL

049780-0003-3

MARKET FACTS WASHINGTON Washington, DC

273090-0001-3

MARKET TRANSFORMATION INST FOR San Francisco, CA

293180-0001-4

MARKLEY $O$ W

Housston, TX

$273100-0001-3$

MARLIN CHAMBER OP COMMERCE Marlin, TX

273110-0001-7

MARQUETIE UNIVERSITY

Biology Dept

Mitwaukee, WI

$049870-0201-9$

MARS ELECTRONICS

Murphysbora, IL

$273120-0001-0$

MARSH PR ROCKEY

Portland, OR

MARSH VALLEY JOINT SD $\$ 21$ Arimo, 1D

$277620-000 t-1$

MARSHAUS MARVIN

Defiance, $\mathrm{OH}$

$266790-0001-7$

MARSING JOINT SCHOOL DIST $\# 363$ Marsing, ID

MARTIN CONSTRUCTION CO Thermopolis, WY

MARTIN MARIETYA CORP Denver, CO

$273130-0005-4$

Space * Mitssile Syst Org

Los Angeles, CA

MARTEN MARIETTA ENRGY SYST INC Oak Ridge, TN

Paducab, KY

$283660-0001-\mathrm{X}$

$286210-0001-6$

$050030-0002-2$

$050030-0604-7$

$$
\text { Chapel Hill, NC }
$$

050050-0001-1

$050050-0003.8$

$281980-0001-1$ 

Awardee
Identification coxle

MARTINEZ CITY OE

Martinez, CA

$288590-0001-7$

MARTINEZ EDGAR R Springfield, VA

MARTINEZ MARIO J

San Juan, RQ

MARTINEZ VINCENTE T

Agena, Guam

$291390-000 ! x$

$273150-00001-1$

MARYLAND ENVTRONMENTAL SVC Annapolis, MD

$281550-0001-X$

$273210-000]-\mathrm{X}$

MARYLAND ROREST \& PARK SERVICE Antapolis, MD

273220-0001-3

MARYLAND PUBLIC TELEVISION

National Productions

Owings Mills, MD

$285070-0101-7$

MARYLAND STATE OF

Energy Admin

Annapolis, MD

Housing \& Compr Dev Dept of Crownswilte, MD

$050290-090]-9$

Maryland Energy Office

Baltimore, MD

MARYLAND UNIV OF EASTERN SHORE

Natural Sciences

Princess Anthe, MD

MARYLAND UNIVERSITY OF

Policy Sciences Dept

College Park, MD

MASEC CORP

Bloomington, IL

Y Complex

Minneapolis, MN

$285250-0101-5$

MASIKO PETER Miami, FL

MASLONKA \& ASSOC INC Phoenix, AZ

MASLUSK」\& LITVAN ARCHTTECTS Springfield, IL

MASON \& HANGER

Burlington, IA

MASON DLXON FARMS

Geltysburg, PA

$050300-1704-6$

$273230-0001-7$

$273230-0102-1$

273240-(1001-0

$292830-8001-3$

$273250-0001-4$

$273260-0001-8$

MASON KOLEHMAINEN RATHBURN \& W Chicago, IL

$287140-6000]-6$

MASSACHUSETTS COMMONWEALTH OF

Boston, MA

050470-0002-8

Cambridge, MA

Conmittee On Criminal Justice Bosion, MA

Communities \& Develop Exec Ofi Boston, MA.

Communities \& Development Bosion, MA

EOEA Office of Tech Assistance Boston, MA

050470-0004-4

$050470-0102-4$

$050470-0202-0$

$050470-1102-X$

$050470-1502-5$

Economic Affairs Exec Off of Boston, MA

$050470-1302-2$
Awardee

Identification code

Energy Resounces Division of

Boston, MA

$050470-1402.9$

Energy Resources Exec Oft

Boston, MA

$050470-0302-7$

Lomg Range Planning Div

Boston, MA

$050470-0402-3$

Mass Enerty Office

Boston, MA

$050470-0502-x$

Mass Health Research Inst line

Boston, MA

$050470-0602-6$

Public Utillites Dept

Boston, MA

$050470-0802-9$

Solar Dept

Bosion, MA

$050470-0902-5$

State Blds Code Commission

Boston, MA

$050470-1202-6$

MASSACHUSETTS GENERAL HOSPITAL

Bostor, MA

$05043000001-5$

MASSACHUSETTS INST OF TECH

Lexinglon, MA

$050440-0002-7$

Trenton, NE

$050440-0003-5$

Electric Dept

Cambridge, MA

$050440-5001-6$

Energy Lab

Cambridge, MA

$050440-5201+9$

Fuid Mexhanies

Cambridge, MA

$050440-5101-2$

Heallh Sciences \& Tech Dir

Cambridge, MA

$050440-1201-7$

Health Sciences Dir

Lexington, MA

Nutrition \& Food Schence Dept

Cambridge, MA

$050440-0702.1$

Systents Dynamic Group

Cambridge, MA

050440-2701-4

050440-530L-5

MASSACHUSETTS LOWELL UNIV OF

Research Foundation

Lowell, MA

MASSACHUSETTS SCI \& TECH

Wakefiteld, MA

$239230-0401-X$

MASSACHUSETTS UNIVERSITY OF Amberst, MA

$273300-0001-9$

MAT.SU COMMUNITY COLLEGE

Palmer, AK

050480-0001-3

MATER DEI HYGH SCHOOL

New Monmouth, NJ

$273310-0001-2$

MATERLAL INTEGRITY SOLUTIONS

Berkeley, CA

MATERLALS \& ELECTROCHEMICAL

Tucson, AZ

128900-0001.X

MATHTECH INTERNATIONAL INC

Falls Church, VA

289410-0001-7

MATRIX DESIGN \& ENGINEERING

Hot Springs, MT

$273320-000 \mathrm{~L}-6$

MATRTX ENGINEERENG INC

Baltimore, MD

273330-0002-8

Pittsburgh, PA

$273330-0001-X$ 
Awardee

MAU WIND ELECTRIC Haikz, HI

MAUELL CORP Dillsburi, PA

MAURER ENGINEERING INC Houston, TX

MAIJRER WARREN W Charlotte Ambsic, VI

MAX I DERBES CONSULTANTS Metairic, LA

MAXIMA CORP Hayward, CA

MAXTEK INC Torrance, CA

MAZER CORP Dayton, OH

MBA ASGOC San Ramon, CA

MBX SYSTEMS INC Tucser, AZ

MCADAMS TECHNOLOGY INC Fairfax, VA

MCAFEE SOFTWARE INC Golden, $\mathrm{CO}$

MCBRIDE ENE Fait Lawn, NJ

MCBRTDE MICRO SOURCE

Albuquerque, NM

MCCALL-DONNELLY JS DIST \#421 MoCall, ID

MCCALLUM JOHN Worthington, $\mathrm{OH}$

MCCANTS THOMAS W Animas, NM

MCCOLL JR JOHN R Branford, CT

MCCOLL ROBERT W Lawrence, KS

MCCRACKEN HORACE Alturas, CA

MCDERMOTT TECHNOLOGY INC

Alliance, $\mathrm{OH}$ Lynchburg, VA

MCDONNELL DOUGL AS CORP Sarla Mortica, CA

Astronantics St Louis, MO

Entrgy Systems St Charles, MO

MCDOWELL-WELLMAN ENG CO Cleveland, $\mathrm{OH}$

MCDS INC Lakewond, $\mathrm{CO}$ Sidney, NE

MOGRATH COMMUNITY LIBRARY MoGralh, AK

MCGRATH ED College, AK
Identifieation code

282310-0001-1

$285940-0001-4$

$050710-0001-6$

$273360-0001-0$

$283110-0001-8$

$050760-0007-3$

$273370-0001-4$

$273380-4001-8$

$273390-0001-1$

$284030-0001-4$

$291240-0001.9$

$28490000001-8$

O LLC

$292490-00014$

$161400-0002-5$

$283690-0001-0$

273400-0001-1

$267620-000]-0$

$273410-0001-5$

$273420 \cdot 0001-9$

$269410-0001-5$

$2854100001 \cdot \mathrm{X}$ 285410-0002-8

$050980.0003-3$

$050980.0302-4$

$050980-0201-\mathrm{X}$

273430-0001-2

$251110-0002-\mathrm{X}$ $251110-0003-8$

$265830-0001-6$ $265820-0001-2$
Awardee

Identification code

MCGRAW HIIL INFO SYSTEMS

Silver Spring, MD

$051060-0001-7$

F W Dodge Div

Washington, $D C$.

051060-0202-8

MCGRAW-EDISTON CO

Power Systems Diy

Canensburg, PA

New Orleans, LA

MCI WORLDCOM

Dallas, TX

MCKEE ARTHUR G

Argonne, IL

Chicago, Il

MCMANIS ASSOC INC

Washington, DC

MCMANUS SCOTT

Cashmere, WA

MCMASTER UNIVERSITY

Hamilton, Canada

MCWHORTER EDWARD D

Ciarts Heights, CA

MDCs

Ralston, NE

$051070-0101-7$

$051070-0109.2$

$292850-0001-0$

$051150-(0) 02-4$

051150-0003-2

$051230-0001-1$

273440-0001-6

288390-0001-1

273450-0001-X

$122670-0002-4$

MilH INDUSTRIFS

Monrovia, CA

MEASUREMENTS ANALYSIS CORP

Torance, CA

$273460-0001-3$

$273470-0001.7$

MECHANICAL CONTRACTNNG IND

Dev Fund of Central Iowa

Des Moines, IA

$273480-0101.7$

MECHANICAL SYSTEMS INC

Choyenne, Wr

$292740-0001-4$

MECHANICAL TECHNOLOGY INC Albany, NY

$051350-00046$

MEDEX ASSISTANCE

Timonium, MD

MEDICAL COLLEGE OF GEORGIA

Physiology Dept

Augusta, GA

$291600-0001-5$

MEGA ENGINEERING

Silver Spring, MD

Washington, DC

$051440-0101-7$

$051480-0001-5$

$0514800002-3$

MEGASOFT TECHNOLOGIES INC Dayton, $O H$

$284280-0001-8$

MELBA JOINT SCHOOL DIST \#136 Melba, ID

$283540-0001-\mathrm{X}$

MELLARD GEORGE La Verne, CA

$267990-0001-4$

MELOY LABS

Springfield, VA

$273500-000 !-4$

MELPAR INC

Falls Church, VA

$273510-000 t-8$

MELTEC ASSOC INC

Shorevicw, MTN

273520-0001-1

MEMBRANE TECHNOLOGY \& RES ANC Menlo Park, CA

051540-000t-3 

Awardee
Identification code

MEMPHIS STATE UNTVERSTTY

Chemistry Dept

Memphis, TN

MEMPHIS UNIVERSITY OF

Chemistry Dept

Memphis,

MENDEZ INC

Cheyenne, WY

Loveland, $\mathrm{CO}$

Prospect Valley, $\mathrm{CO}$

MENKES MUNICTPAL SERVICE INC

West Orange, NJ

MEPPSCO INC

Boston, MA

MERADCOM

Fort Belvoir, VA

MERCFR COUNTY OF

Energy Development Board

Haten, ND

MERCO INC

Golden, $\mathrm{CO}$

MEREDTTH \& FINLAYSON

Ontario, Canada

MERIDIAN INSTITUTE

Dillon, $C O$

MERYLLAT DBA MIDWEST FDN

Topeka, KS

MERFT MACHINERY INC

Pittsburgh, PA

MERIZO SHORES LTD PARTNER San Francisco, CA

$051750-0101-9$

284040-0101-4

254440-0003-2

254440-0004-0

$254440-0002-4$

$273530-0001-5$

$273540-0001-9$

$273550-0001-2$

$051910-0101-X$

$287850-00001-9$

$052150-0002-6$

$291360-0001.9$

286180-0001-9

273560-0001-6

MERRL MARK

Willow, AK

273070-0001-6

MERRILL NATURAL RESOURCES INC

Richmond, VA

MERUTT INC

Santa Monica, CA

META ENVIRONMENTAL INC

Walertown, MA

META SOLUTONS INC

Linthicum, MD

METAL PROPERTIES COUNCIL

New York. NY

METCALF \& EDDY

Wakefield, MA

METHACTON SCHOOL DISTRICT Eagleville, PA

273580-0001-3

$285360-0001-5$

$284290-0001-1$

273590-0001-7

$264920-00001-3$

$052260-0002-2$

METOROLA

Phoenix, AZ

METRICS INC

Allanta, GA

METRO

Seattle, WA

METRO MECHANICAL INC

Phoenjx, AZ

METRO SANITARY DISTRICT

Chicage, IL

$273620-0001-4$

$254760-0001-1$

$273630-000 \mathrm{l}-8$
$273570-0001-\mathrm{X}$

$264970-0001 \cdot 1$

$$
\text { Awardet }
$$

Identifteation

code

METRO STUDY CORP

Washington, DC

$273640-0001-1$

METROPOLITAN WASH COUNCIL GOV

Wașington, DC

273660-0001-9

METROPOLITAN WASH PLAN \& HOUS

Washington, $\mathrm{DC}$

$273650-0001-5$

METROTECH

Dallas, $T X$

$273670-0001-2$

MHP ELECTRTC

Middlesboro, $\mathrm{KY}$

$290360-0001-7$

MICHAEL A MOORE \& ASSOC

Atlanta, GA

MICHAELS ENGINEERING

New Haven, CT

$052570-0004-0$

MICHAELS FERSCHL

Milwaukee, WI

MICHIGAN ENERGY RSRC RES ASSN

Detroil, MI

MICHIGAN MOLECULAR INSTITUTE

Midjland, MII

$273790-0001-2$

MICHIGAN PUBLIC POWER

Traverse City, MI

$273800-0001-2$

MICHIGAN STATE OF

Commerce Energy Admin Dept

Lansing, MI

$052610-1202-3$

Family Independence Agency

Lansing, ML

$052610-1102-7$

Labor Dept of

Lansing, MI

$052610-1302-\mathrm{x}$

MICHIGAN 'TEGHNOLOGICAL UNTV

Metallurgical \& Materials Eng

Houghton, MT

052640-1001-3

MICHIGAN UNIVERSTTY OF

Nac Eng \& Radiohogical Science

Ann Arbor, MB

$052650-3301-7$

MICOMAGE

Arlington, VA

$273810-0001-6$

MICRAMICS INC

Santa Clara, CA

$289510-0001-\mathrm{X}$

MICRO COATING TECHNOLOGLS

Chamblee, GA

289480-0001-2

MTCROAGE FEDERAL

Vienna, VA

$287640-000]-X$

MICROBAC LABS

Pittsburgh, PA

MICROCARBURETOR CORP

Buffalo, NY

MICROECON ASSN

Stanford, CA

MICRON ELECTRONICS INC

Meridian, ID

MICRONIC INC

Miami, FL
MICROPYRETICS HEATERS INTL INC

Cincinnati, OH

MICROSOFT CORP

Charlotle, NC
$107850-0002-\mathrm{X}$

273820-0X01-X

$273830-000]-3$

$257720-0003-9$

288020-0001-8

290040-0001-]

$259990-0002-5$ 


$$
\begin{gathered}
\text { Identification } \\
\text { code }
\end{gathered}
$$

MICROWAVE DATA SYSTEMS INC

$$
\text { Rochester, NY }
$$

$223290-0002-2$

MID ATLANTIC CORP

Bethesda, MD 273840-0001-7

MII MISSOURI COUNCIL OF GOVTS

$$
\text { Jefferson Cily, MO }
$$

MID-AMERICA CONSULTING GROUP

Miamisburg. OH

$254370-0001-4$

MID-ATLANTIC SOLAR ENERGY ASSN

Philadetphia, PA

$052750-0001-4$

MID-COLUMBLA LIBRARY

Kenmewick, WA

MID-CONTINENT EARTH STATIONS

New Ujderwood, SD

MIIDDLE EARTH FARMS

Louisturg, MO

MIJDLE RIO GRANDE CONSERV DIST

Albuquerque, NM

Los Alamos, NM

273880-0001-1

$273890-0001-5$

2668860-0001-9

MIDDLE TENNESSEE STATE UNIV

Murtreasboro, TN

MTDDLE WEST SERVICE CO

Chicago, IL

MIDDLEBURY

Middlebury, VT

MIDLAND ROSS CORP

Columbus, $\mathrm{OH}$

Toledo, $\mathrm{OH}$

MIDVALE SCHOOL DISTRICT $\$ 433$

Midvale, ID

$052840-0001-3$

$052840-0002-1$

$052870 \cdot 0001-4$

$273900-0001-5$

282970-000]-X

273910-0001-9

$273910-0002-7$

MEWAY PLUMBENG INC

Albany, $O R$

$283710-0001-4$

MIDWEST CONTROL SYSTEMS INC

Coral Springs, FL

$265690-0001-4$

MIDWEST ENERGY EDUCN CONSORTM

Redfield, IA

MIIDWEST MICRO

Flercher, $\mathrm{OH}$

MIDWEST POWER SYSTEMS INC

Des Moines, IA

MIDWEST REGIONAL SOLAR VENT

Esgan, MN

MIDWEST SUPERCONDUCTIVITY INC

Lawrence, KS

MIKE J THIEL INC:

New Madrid, MO

MIL CORP

Bowie, MD

MILCOM SYSTEMS CORP

Virginia Beach, VA

MHLES JOHN

U⿱宀㠯丿, Ja, IL

MILLARD SCHOOL DISTRICT

Delta, UT

MILLCREEK ENERGY

Heardsburg, CA

MILLER \& LENTS LTD

Houston, TX

$052990-10002-2$

$264960-0001-8$

$211300-000 t-\mathrm{X}$

273920-000t-2

283920-0001-3

053020-0015-6

$178270-0002-9$

$286070-0001-2$

273930-0001-6

$053110-0001-5$

$273940 \cdot 0001-\mathrm{X}$

$2652200-0001-6$

$$
\text { Awardee }
$$

Identification

code

MILLER DONALD R

Rosean, MN

273950-000]-3

MILLER DR MARVIN $M$

Newhon, MA

$273960-0001-7$

MILLER JAMES A

Kearney, NE

MILLER MARK A

Juneau, AK

MLLLER THOMAS J

Kodiak, AK

MILLS JR JOSEPH L

Jamettsville, MO

MILSTEN DONALD ELLIS

Baltimore, MD

MIILTEL INC

Herndon, VA

MINARD RUN EXPLOR CO INC

Bradford, PA

MIND TECH LLC

Evergreen, CO

MINERAL AREA COLLEGE

Flal River, MO

MINERAL EXPLORATION DRLLL

Fort Collins, CO

Hanna, WY

MINGES ASSOC INC

Avon, CT

MINGO ROOER

Washington, $\mathrm{DC}$

MRICARS INC

Goleta, CA

MINIDOKA COUNTY OF

Sheriff's Office

Rupert, ID
MINING \& RECLAMATION COUNCII Washington, DC

MINN DR HOWARD S

Lake Charles, LA

MINNESOTA ENV SCIENCE FDN INC Mintreapolis, MN

MINNESOTA STATE OF

Economaic Security Dept of St Paul, MN

Public Service Dept of St Paul, MN

MINNESOTA UNIVERSLTY OF

Research Tech Transfer Admin Minneapolis, MN

Sponsored Projects Adrain Míneapolis, MN

MINOR WILBERT R

Newark, DE

MINORITY ACCESS INC

Hyaltsville, MD

MINORITY OWNED BUS TECH TRANSF

Falls Church, VA

Washington, $D C$ $270410-0001-x$

$273040-0001-5$

$267600-0001-3$

271040-0001-1

$265620-0001-7$

$291260-0001-6$

$273970-0001-0$

$274840-0005-9$

$282860-000 \mathrm{j}-3$

273980-0001-4

$273980-0002-2$

$273990-0001-8$

$277690-000[-7$

$274000-0001-2$

$168550-0101-7$

274010-0001-6

265170-0001-1

274020-0001-X

053480-0201-J

053480-1601-2

$053490-3301-8$

053490-3201-1

282250-0001-3

$293190-0001-8$

2839100001-X

283910-0002-8 

Awardee
Identification
code

MINORITY SERVICES INC

Silver Spring, MD

274030-0001-3

MIRACAL SERVICES INC

Hanoyer, MD

$291610-0001-9$

MISSISSIPPI COUNTY

Arkanses Epe Inc

Blytheville, AR

Nom-Profit Corp

Blytheville, AR

MISSISSIPPI FUEL ENGY MGMT Jackson, MS

MISSISSIPPI GEO ECO TOPO SVY

Jacksson, MS

MISSISSIPPI STATE OF

Fuel \& Energy Management Jackson, MS

Human Services Dept of Jackson, MS

MISSOULA COUNTY OF

Missoula, MT

Missoula County Courthouse Missoula, MT

MISSOURI FOREST PRODUCTS ASSOC Jefferson City, MO

MISSOURI FRIENES OF THE EARTH Columbia, MO

2865]0-0101-0

$286510-0201.7$

COM

$274040-0001-7$

$274050-0001-0$

$053710-0701-6$

$053710-0601-x$

$274060-0001-4$

274060-0101-0

274080-9001-1

MISSOURI UNIVERSITY OF

Curators

Rotla, MO

Engineering Department of Columbia, $\mathrm{MO}$

$274090-0001-5$

Nuclear Engineering Dept Rolla: MO

$053810-2302-2$

$053810-2601-3$

053810-1702-2

Rock Mech \& Explos Res Ctr Rotla, MO

053810-2502-5

MISSOURI-ROLLA UNIVERSTTY Rolla, MO

$274070-0001-8$

MITCHELL RICHARD S Jonesboro, AR

MITRE CORP

Bedfotd, MA

Boston, MA

MeLean, VA

Washinglon, DC

Euergy Conversion Dept

Washington, $\mathrm{DC}$

Metrek Div

McLean, VA

Westgate Research McLean, VA

MITRE TEK SYSTEMS INC Mclean, VA

MitTtelhauser CORP

Chicago, IL

$277340-0001-0$

$053860-00001-2$

$053860-0003-9$

$053860-0002-0$

053860-0004-7

053860-0404-2

053860-0502-2

053860-0602-9

$287380-0001-6$

053880-00003-6

MUS MANUFACTURING ENTERPRISES Gilbert, $\mathbf{A Z}$
$293280-0001-7$
Awardee

Identification code

MK-FERGUSON CO

Power Div

Albuquetque, NM

MK-FERGUSON OF DAK RIDGE CO

Oak Ridge. TN

I40340-0103-8

MOAPA BAND OF PAIUTES

Moapa, NV

MOHAY CHEMICAL CORP

Pittsburgh, PA

MOBIL RESEARCH \& DEV CORP

New York 1 NY

Paulsboro, NJ

Princelon, NJ

Research Dept

Paulsboro, NJ

$282950-0001-2$

$274100-0001-5$

$274 \times 10-0001-9$

$053930-0003-0$

053930-0001-4

053930-0002-2

MOBIL TYCO SOLAR ENERGY CORP

Waltharn, MA

053930-0101-0

MOBILE CHARACTIZATION SERV LLC

Carlsbad, NM

MOBLE SOLAR ENERGY CORP

Waltharn, MA

$284990-0001.0$

MOBIUS MANAGEMENT SYSTEMS INC

Clinton, MD

Falts Church, VA

New Rochelle, NY

$223370-0001-\mathrm{X}$

$223370-0003-6$

$223370-0002-8$

MOBLESOURCE INDUSTRIES INC

Indiana, PA

MODERN ALLOYS INC

Stanion, CA

MODERN ELECTRIC POWER

Boston, MA

$290560-0001-2$

$274130-0001-6$

MODERN PROCEDURES INSTTFUTE OF

Washington, DC

MODESTO IRRIGATION DIST

Modesto, CA

MODLANA \& ASSOC

Washington, Italy

MOFFAT CSD

Moffat, $\infty O$

MOFFITT REPORTING ASSOC

Vienna, VA

MOHAVE SOLAR ENERGY COMM

Kingman, AZ

MOHEGAN KaDS aH CLUB

Woonsocket, RI

MOLECULON RESEARCH CORP

Carnbridge, MA

MONTTOR LABS INC

Englewood, $\mathrm{CO}$

San Diego, CA

MONMOUTH SCHOOL DEPARTMENT

Richmond, ME

MONOLITHIC SYSTEMS CORP

Englewood, $C O$

MONOSOLAR INC

Santa Monica, CA

MONROE WAYNE W

Windsor, $C O$

$040050-0001-0$

$178280-000]-4$

$274150 \cdot 0001 \cdot 3$

$274160-0001 \cdot 7$

$05402000003-4$

$274170-0001-0$

$274180-0001-4$

$274190-0001-8$

171880-0002-6

171880-0001-8

$274200.0001-8$

$274210-0001-1$

$054220-0001-3$

2742200-0001-5 
Awardee $\begin{gathered}\text { Identification } \\ \text { code }\end{gathered}$

MONSANTO RESEARCH CORP

Daylon, $\mathrm{OH}$

054340-0000]-3

MONTANA COLL OF MINERAL SCI

Butte-Silver Bow, MT

MONTANA ENERGY

Butte, MT

MONTANA STATE OF

Admín Dept

Helena, MT

Environmental Quality Dept of

Helena, MT

Fish Widdife \& Parks Dept

Kałispell, MT

Missoula, MT

Mines \& Geology Burean of

Butte, MT

Pubjic Health \& Honuan Services Helena, MT

Supplemental Dept

Helena, MT

Weatherization Dept

Helena, MT

MONTANA UNTERSITY OF

Admin Dept

Helena, MT

MONTEFIORE COMP HEALTH New' York, NY

MONTEFIORE HOSPTTAL \& MEDICAL New York, NY

MONTGOMERY ENGINEERING

Watertown, SD

MOORE KENNETH V

Aiken, SC

MOORE LARUE \& SCHAFER

Dallis, TX

MOORHEAD CITY OF

Planning Dept

Moortend, MN

MORALES \& ASSOC LLC Ojai, CA

$274230-0001-9$

$054420-1102-9$

$054420-1302-1$

054420-1203-3

054420-1204- I

$054420-1701-9$

$054420-1402-8$

$054420-1502-4$

$054420-1602-0$

054440-0202-7

274240-000t-2

274250-0001-6

194040-0002-7

2015620-0001-1

272000-0001-9

$274280-0101-3$

$260260-0001-1$

MORGAN COMMUNITY COLEEGE

Forl Morgan, CO

MORgAN SAMUELS CO INC

Beverly Hills, CA

$274290-0001-0$

MORGANTOWN UTILITY BOARD Morgantown, WV

MORPAC INDUSTRIES INC Tucson, AZ

MORRIS F DE CASTRO CLINIC

Heallh Dept Charlote Amalie, VI

MORRIS GURALNICK ASSOC

San Francisco, CA

MORRIS SCOTT $W$ Santa $\mathrm{Fe}, \mathrm{NM}$

MORRISON-KNUDSEN CO INC

Power Systems Division

Rocky Mount, NC

$290940-0001-6$

$189860-0002-4$

$2743000-0101-7$

$274310-0001-4$

274330-0001-1

055040-0103-X
$054400-0002-X$

Awarilee

Identification code

MORSE CHALN CO

Jthaca, NY

MOSCOW JOINT SCHOOL DIST \$281

Moscow, ID

MOSCOW-LATAH COUNTY LIBRARY

Moscow, ID

$274360-0001-2$

MOSLAC MAGING TECHNOLOGY INC

Palaine, IL

MOSLER GOVERNMENT SALES

Alexandria, VA

MOSTEK CORP

Carroliton, TX

MOTHER GOOSE NURSERY

Winslow, ME

MOTLEY CONST CO

Carmichael, $\mathrm{CA}$

MOTOR COACH INDUSTRIES

Pembina, ND

MOTOR VEHLCLE MFRS ASSN

Detroit, MI

MOTORCARBON LLC

Mianisburg, OH

MOTOROLA INC

Lawrence, KS

System Solution Group

Golden, $\mathrm{CO}$

MOTT COMMUNITY COLLEGE

Fint, MI

MOTT CORP

Farmington, $C T$

MOUNT HOOD NATIONAL FOREST

Greshath, $O R$

MOUNTAIN BELL TELEPHONE

Albuquerque, NM

MOUNTAIN COMM WORKSHOP

Berea, KY

$289180-0001-4$

170950-0003-4

$274370-0001-6$

$274380-0001-X$

$274400-0001-3$

$288140-0001-8$

$1158100001-4$

284450-000t-2

055260-0018-4

055260-0919-X

$2744200001 \cdot 0$

$292730-0001-0$

$274430-0001-4$

$055650-0001.7$

MOUNTAIN MANOR CONDO ASSOC

Wharton, NJ

MOUNTAIN REGION CORP

Flagstaff, AZ

MOVEMENT INC

Phoenix, AZ

MOWERY JAMES W

Oldsmar, FL

292930-0001-6
MPA MODULAR L.LL Arlinglon, TX

MPI SOFTWARE TECHNOLOGY INC Starkville, MS

MR WIZARD STUDIO

Canoga Park, CA

MS TECHNOLOGIES CORP

Gaithersburg, MD

MSA C/O GILSON ENGR SALES INC Pittsburgh, PA.

MSA RESEARCH CORP

Evans Cily, PA

MSP CORP

Minneapolis, MN
$274440-0005.8$

286640-0001-8

107920-0003-X

$293100-000$ t-5

270530-0001-X

279530-0001-6

284300-0001-1

$274450-00001-1$

267780-0001-5

260160-0001-9

2744600-0001-5

$293310-0001-4$ 
Identification
code

MT PUB SERVICE COMM

Helena, MT

MTS TECHNOLOGIES INC

Alexandria, VA

Arlington, VA

$274470-0001-9$

$287170-0002-5$

287170-0001.?

MUEHLECK NORMAN

Rock Island, WI

274480-0001-2

MUELLER ASSOC INC

Baltimore, MD

MULDOON CENTER

Rockford, IL

MULTILINK TECHNOLOGY CORP

Santa Monica, CA

MURDOCK JAMES D

Moylan, PA

MURPHY EDITH

Alexandria, VA

MURPHY MARVIN W

Reno, NV

MURRAY JOHN R

Limerick, ME

MURTAUGH JOINT SCH DIST \#418

Murtaugh, JD

MUSEUM OF SCIENCE INC

Bosion, MA

Miami, FL

MUSKEGON MERCY HOSPITAL

Muskegon, MI

MUSON GLENN W

Pawlet, VT

MYKOTRONX INC

Colurabia, MD

MYRL \& ROY'S PAYING INC

Sioux Falls, SD

055850-0001-2

$274490-0001-6$

$283930-0001-7$

$270450-0001-4$

$291440-0001-4$

$273170-0001-9$

$270890-0001-X$

$165020-0001-0$

$222790-00001-6$

$222790-0002-4$

2828370-6001-7

$268210-0001-8$

$288730-0001-0$

$286840-0001-3$

$\mathbf{N}$

N CASTLE CITY VOC SCHOOL

Wikmington, DE

$274510-0001-X$

NAC INTERNATIONAL

Nerctoss, GA

$265240-0001-3$

NACRE

Washinglon, DC

NADLER MOBILE OFFICES Kingston, NJ

NAHB RESEARCH FOUNDATION Rockville, MD

Special Sudies Rockville, MD

NAJAR CONSTRUCTION

Las Cnuces, NM

$291180-0001-0$

$292510-0001-8$

056190-0001-X

056190-0201-2

NANO INSTRUMENTS INC

Oak Ridge, TN

$284620-000 \mathrm{t}-7$

NANOPOWDER ENTERPRISES INC Piscataway, NJ

NANTICOKE MEMORIAL HOSPITAL

Facilitites Dept

Seaford, DE

$056220-0101-3$
Awardee

Identification coude

NARAMORE BAIN BRADY

Seattle. WA

$274580-0001-5$

NASA

Mountain View, CA

$056300-0012-8$

Pasadena, CA

$056300-0030-1$

Admin

Langley Res Center, VA

$056300-2408-6$

Jet Propulsion Laboratory

Pasadena، CA

$056300-0410-7$

Radiation Sciences

Hampion, VA

$056300-3113-9$

NATHAN ASSOC INC

Washìngton, $D C$

$274610-0001-2$

NATIONAL ACADEMY OF ENGNG Arlington, VT

274890-0(0)1-7

NATIONAL ACADEMY OF SCIENCES

Washington, DC

056450-000]-3

Bidg Tech Center for

Washington, DC

056450-2201-7

National Research Council

Wastington, DC

056450.1101 .5

NATIONAL ARCHIVES \& RECORDS AD

College Park, MD

2902900001.5

NATIONAL ARCHIVES \& RECORDS SR Golden, $\mathrm{CO}$

27462000001-6

NATIONAL ASSN OF COUNTIES

Washingion, DC

$274900-0001-7$

Research Foundation

Washinglon, DC

$274900-0101-3$

NATIONAL ASSN OF CTYS RSCH Washington, DC

$056510-0001-1$

NATIONAL ASSN OF FARMWORKERS

Washington, DC 2668500001-5

NATIONAL ASSN OF STATE ENGY OF

Alexandzia, VA

286250-0001-0

NATJONAL ASSOC FOR EQUAL OPPOR Silver Spring, MD

289620-0001-6

NATIONAL ASSOC OF COMMUN RSCH Washington, DC

$274640-0001.3$

NATIONAL BUREAU OF STANDARDS

Gaithersburg, MD

Washinglon, DC

004460-0004-3

$004460-0002.7$

Analytical Chemistry Center

Gaithersburg, MD

$004460-0204-6$

Bnilding Center For Tech

Gaithersburg, MD

Building Equip Div

Gaithersburg, MD

Electricity Div

Gaithersburg, MD

Washington, DC

Governiment Acconints

Washington, DC

Materhals Sclente Center

Gaithersburg, MD

$004460.1304-8$

$004460-0104-X$

004460-0404-9

004460-0402-2

$004460-1602-0$

Mech Enpineering Dept

Gaithersburg, MD

$004460-0304.2$

$004460-14044$ 
Awardee

Nuclear Radiation Div

Gaithersburg, MD

Quantam Metrology Group

Gaithersburg, MD

Rodiation Theory Group

Gaithersburg. MD

Standard Reference Data

Washington, DC

Temperature \& Pressare Div

Gaithersburg, MD

NATIONAL CANCER INSTITUTE

Rockville, MD

NATIONAL CAPITOL PLANNING CO

Washington, DC

NATIONAL, CARPOOL ASSN

Porlland, OR

NATFONAL, CENTER RESEARCH RECOV

Washington, DC

NATIONAL CLEAN AIR COALITION

Washington, DC

NATIONAL COMMUNITY ACTION FDN

Washington, LC.

NATIONAL CONF STATS BLDG

McLear, VA

Tate Legislature

Denver, $C O$

NATIONAL CONSRTM BLK PROF DEV Louisville, KY

NATIONAL, CONSUMER LAW CENTER Boston, MA

NATTONAL, COUN ON THE AGING INC

Washington, DC

280470-0001-2

274670-0001-4

$274670-0102-9$

274930-0001-8

175150-0002-3

274600- $0001-9$

NATIONAL COUNCIL ON RAD PROTEC

Bethesda, MD

195590-0001-2

NATIONAL CRANES OF OKLAHOMA

Oklahoma City, OK

$290780-0001-5$

NATIONAL CTR RES RECOVERY

Washinglon, DC

274650-000I-7

NATIONAL ECON RES ASSOC INC

Boston, MA

056830-0003-3

Los Angeles, CA

New York, NY

056830-0004-I

056830-0001-7

Seattle, WA

NATIONAL ELECTRICAL SYSTEMS

Boonville, NY

$056830-0002-5$

NATIONAL ELEC High Point, NC

NATIONAL ENERGY CORP

Glen Ellyn, IL

285860-000]-9

NATIONAL ENV

Washington, DC

NATIONAL FAMILY OPINION

Northwood, OH

NATIONAL FENESTRATION CO

Topeka, KS

NATIONAL FISHERY RESEARCH CTR

Seatte, WA
Awardee

kdentification code

NATIONAL FOOD PROCESS ASSN

Dublin, CA

056900-0002-7

Western Research Laboratory

Berkeley, CA

NATIONAL FUEL GAS SUPPLY

Buffalo, NY

NATIONAL GENERAL SUPPLY Great Falls, MT

$056900-0101-5$

$274750-0001-X$

$287610-0001.9$

NATIONAL GEOPHYSICAL DATA CTR Boulder, $C O$

287450-0001-8

NATTONAL GOVERNORS ASSN

Washington, DC

056940-000]-3

Best Practices Center for

Washington, DC:

NATIONAL GOVERNORS CONF

Washington, DC

056940-0101-X

NATIONAL HOMES MFG CO

Lafayette, $\mathbf{N}$

$274760-0001-3$

$274770-0001-7$

NATTONAL INDIAN YOUTH COUNCIL Albuquerque, NM

NATIONAL INST FOR COMM DEVEL

Arlington, VA

Washington, DC

274590-0001-9

$274590-0002-7$

NATIONAL INST OF STNDS \& TECH Washinglon, DC

I80110-0005-5

NATIONAL JOURNAL GROUP

Washington, DC

291480-0001-9

NATIONAL LEAD COMPANY OF OHIO

Cincinat, $\mathrm{OH}$

$274790-0001-4$

NATIONAL LEAGUE OF CITIES

Mayors Conf on

Washington, DC

$057050-0101-0$

NATIONAL LIME ASSN

Washington, DC

NATIONAL OPINION RES Chicago, iL

$274800-0001-4$

274810-0001-8

NATIONAL PUBLIC SER RESEARCH Afexandria, $V A$

274970-0001-2

NATIONAC RECREATION \& PA Arlingion, VA

274820-0001-1

NATIONAL RESEARCH DEVEL CORP

London. United Kingdom

274980-0001-6

NATIONAL RESOURCES DEFENSE Washington, DC

$274850-0001-2$

NATIONAL RESTAURANT ASSOC Washington, DC

$274860-0001-6$

NATIONAL SAVINGS \& LOAN

Washington, DC

NATIONAL SCIENCE FOUNDATION

Washington, DC

Eavey, Dr. Cheryl

Arlington, $\mathrm{VA}$

NATIONAL SECURITY AGENCY

Albuquerque, NM

Cheverly, MD

Forl George G Meade, MD

Riverdale, MD
$274870-0001-X$

057130-0001-X

057130-1903-9

$057150-0009-2$

$057150-0010-6$

057150-0003-3

057150-COL 1-4 

Awardete
Identification
code

COMSEC Acquisition \& Mat Mgt

Fort George G Meaje, MD

057150-0103-X

Langley Research Center

Fort George G Meate, MD

$057150-0403-9$

V-25

Fort George $\mathbf{G}$ Meade, MD

$057150-0503-5$

NATIONAL STEEL CORP

Pittsburgh, PA.

NATIONAL SUPPORT SYSTEMS

Tulsa, OK

$274880-0001-3$

274990-0001-X

NATIONAL SYSTEMS \& RESEARCH CO

Montrose, $\mathrm{CO}$

$057190-0007-0$

NATIONAL TRANSPORT POLICY COMM

Washington, DC

NATIONAL URBAN LEAGUE INC

New York, NY

NATIONS BANK

Washington, DC

NATIVE AMERICAN SALES INC

Detwer, $\mathrm{CO}$

Englewood, $\mathrm{CO}$

275000-0001-4

057250-0002-8

288580-0001-3

258700-0604-1 $258700-0003.3$

NATIVE AMERICAN SYSTEMS INC

Englewoud, $C O$

NATL FDN FOR FUNC BRAIN RES

A]tuguerque, NM

NATL INST OF ENV HEALTH SCI

Duntam, NC

292670-0001-2

NATL INSTITUTE FOR ENV RENEWAL MayGeld, PA

NATURAL DYNAMICS

Des Moincs, LA

NATURAL GAS VEHICLE COAL Arlington, VA

NATURAL RESOURCERS CORP Brooklyn Park, MN

$291340-0001-1$

$244100-0001-2$

283410-0001-6

275010-0001-8

290490-0001-0

275020-0001-1

NATURAL RESOURCES DEFENSE CNCL

New York, NY

Palo Alto, CA

San Francisco, CA

Washinglon, DC

Washington Office

San Francisco, CA

057370-0004-4

$057370-0002.8$

$057370-0003-6$

$057370-0001 . \mathrm{X}$

057370-0103-2

NATURE AMERICA INC New Yotk, NY

NATURE CONSERVANCY Arlington, $V A$

NAUTILUS OF AMERICA INC

Berkeley, CA

NAYAJO ENGINEERING \& CONSTR CO Stiprock, NM

NAVAJO NATION

Arlington, VA

288760-0001-]

247690-0002-2

292280-0001-5

$287780-0001-?$

Fish \& Wildife Dept

Window Rock, AZ

$057380-0003-X$

$057380-0402-7$

Navajo/Untra Program

Window Rock. AZ

057380-0502-3

NAVAL RESEARCH LABORATORY

Washington, $\mathrm{DC}$

261970-0001-6

$$
\text { Awardee }
$$

Identification

code

NAVAL SURFACE WARFARE

Bethesda, MD

NAVY DEPARTMENT OF

Corona, CA

[Dahlgren, VA

Goose Creek, SC

Indian Head, MD

Norfolk, VA

Pearl City, HI

Ricbland, WA

Coestal Systems Center

Panama City, FL

IPYARDS Conversion \& Repair

San Francisco, CA

Naval Air Warfare Center

Orlando, FL

Naval Center For Acquisition

Richiand, WA

Napal Ctr for Acquisition Trng

Norfolk, VA.

Naval Ctr for Aquisition Trag

Rock lsland, IL

Naval Fac Enc Command OS

Arlington, VA

Naval Facflities Eng Conntumd Alexandria, VA

Naval Ocean Research \& Dev

Gulfport, MS

Naval Offace of

Washington, DC

Naval Ordnance $L$

Indian Head, MD

Silver Spring, MD

Naval Research Lab

Washington, DC

Naval Research Office of

Arlingtoni $V_{A}$

Washington, DC

Naval Ship Engineribg Center

Philadelphia, PA

Washington, DC

Naval Spawar Systems Cepter

North Charleston, SC

San Diego, CA

Naval Speclal Warfare Gr Two

Norfolk, VA

Naval Surface Wariare Center

Indian Head, MD

Radiation Phy Group

San Diego, CA

Weapons Support Facility

Seal Beach, CA

NAZAR ANDE

Sunderland, MA

283120-0001-1

$05743000075-1$

057430-0027-1

$057430-0070-0$

$057430-0042-5$

057430-0041-7

057430-0074-3

057430-0072-7

057430-8469-6

057430-8821-7

057430-6471-7

$057430-9272-9$

$057430-9041-6$

057430-9376-8

$057430-8202-2$

$057430-5301-4$

$057430-8368-1$

057430-8625-7

057430-8542-0

057430-8523-4

$057430-1425-6$

057430-2402-2

057430-2425-1

057430-1718-2

057430-1725-5

$057430-8973-6$

$057430-8920-5$

057430-8141-7

$057430-5142-9$

$057430-8720-2$

057430-9122-6

275040-0001-9

NBI INC

Rosslyn, VA

NC CONFERENCE BLK MAYORS

Catrbore, NC
057480-0009-1

274570-0001-1 


\section{Awardiee}

ND STATE SCHOOL OF SELENCE

Wahpeton, NE

NEBRASKA MUNICIPAL

Lincoln, $\mathrm{NE}$

NEBRASKA STATE OF

Health Dept

Lincoln, NE

Radiological Heajth Div

Lincoln, NE

NEBRASKA UNIVERSTTY OF

Bjological Sciences School of

Lincoln, NE

NEBRASKA WESTERN COLLEGE

Westem Tech Conty Coll

Scotsbluff, NE

NEEDLE \& ROSENBERG PC

Atlanta, GA

NEEEEY DEREK K

Pittsourgh, PA

NEGRON JUAN RIVERA

Bayamon, $R Q$

NEHER MICHAEL

Missoula, MT

NEIGHBORFOOD HOUSE INC

Seatule, WA

NELSON ROBERT E

Juneau, AK

NENANA PUBLIC LIERARY

Nenana, AK

NEOPOST

Union City, CA

NETPREDICT INC

Menlo Park, CA

NETWORK SOFTWARE ASSOC INC Arlington, $V A$

NETWORLD SYSTEMS

Scottsdale, AZ

NEVADA CHIEDREN'S CENTER

Las Vegas, NV

NEVADA STATE OF

Energy Ofifice

Carson City, NV

Motor Vehicles Dept of

Carson City, NV

NEVADA TAHOE CONSERYATION

South Lake Tahoe, CA

NEVADA TESTTNG INSTTTUTE INC

Las Vegas, NV

NEVADA UNIVERSITY OF

Curriculum \& Education Dept of

Reno, NV

Electrical Engineering Dept

Las Vegas, NV

NEW AGE CONSTRUCTION

Bismarck, ND

NEW CREATTVE COMMUNTCATONE

Corpus Christi, TX
Identifleation

code

$274520-0001-3$

$275070-0001-X$

$057580-0601-7$

$057580-0701-3$

057590-180i-9

$057600-0101-9$

$281120-0001-8$

$264310-0001-3$

$275080-0001-3$

$275090-0001-7$

275100-0001-7

$277510-0001-5$

$275130-0001-8$

$292560-0001-6$

$2932900001-0$

$268970-0001-9$

$28570000001-4$

$2874700001-5$

057770-0501-2

$057770-2301-0$

269580-0001-3

$287460-0001-1$

$057780-2201-8$

$057780-2302-2$

280450-0001-5

$284590000 \mathrm{I}-\mathrm{X}$
Awardee

Identifiestion code

NEW ENERGY CORP OF INDIANA

South Bend, IN

$096550-000 t-\mathrm{X}$

NEW ENGLAND ALTERNATIVE

Brattleboro, VT

NEW ENGLAND AOUARIUM CORP

Edgerton Reseanch Lohoratory

Boston, MA

NEW ENGLAND FUEL DSTITUTE

Boston, MA

$272550-0001-0$

NEW HAMPSHIRE STATE OF

Eneray Conservition Ofifice

Concord, NH

$058040-1301-7$

Governor's Council on Energy

Concord, NH

$058040-120 \mathrm{I}-0$

NEW HAMPSHIRE WATER RESOURCE

Concord, NH

275140-0001-1

NEW JERSEY MARANE \$CI CONSORT

Highlands, NJ

$275150-0001-5$

NEW JERSEY STATE OF

Energy Div

Newark, NJ

$058130-0401.7$

Public Utillties Board of

Newark, NJ

$058130-0201-4$

NEW LIFE CHRISTLAN CENTER

Las Vegas, NV

NEW LONDON COUNTY EXT SVC

Norwich, CT

$283340-0001-4$

NEW MEXICO CONF OF CHURCHES

Albuquexque, NM

NEW MEXICO ENERGY $R$ \& D INST

Albuquetque. NM

$103130-0002-6$

NEW MEXICO INST OF MIN \& TECH

Socono, NM

$058200-0001-3$

NEW MEXTCO INST OF TECHNOLOGY

Los Alamos, NM

NEW MEXICO MESA INC

Los Alamos, NM

275170-0001-2

$224460-0002-9$

NEW MEXICO RESONANCE

Albuquerque, NM

$287070-0001-4$

NEW MEXICO STATE OF

Eeonomic Bevelopment Dept

Senta Fe, NM

$058210-2203-7$

Environnental Improvement Dix

Los Alamos, NM

$058210-1002-0$

Gowernor's Comm Office of

Santa Fe, NM

$058210-2403-X$

Mortgage Funnce Agency

Albuquerque, NM

058210-2301-7

Palice

Albuquerque, NM

$058210-2501-\mathrm{X}$

NEW MEXICO STATE UNIVERSTTY

Waste Mgmt Edu Res Consorthn Las Cruces, NM

NEW MEXICO UNIVERSITY OF

Albuquerque, NM

$058220-1402-\mathrm{X}$

$058240-0001-8$

NEW ORLEANS UNIVERSTTY OF

New Orleans, LA

$058280-0001-2$ 

Awardee
Identification code

NEW SOUTH ASSOC

Stone Mountain, GA

$285050-0001-3$

NEW WAVE COMMTNICATION INC

Washington, DC

275180-9001-6

NEW YORK CITY OF

Honsing Anthority

New York, NY

$058450 \cdot 0701-1$

Traus Dept of / Alt Fued Prog

New York, NY

NEW YORK INSTITUTE OF TECH

New York, NY

058450-0801-8

NEW YORK MEDICAL COLLEGE

Microbiology Dept

New York, NY

NEW YORK STATE COLLEGE OF

Agriculture / Cornell Univ

Ithaca, NY

$058490-0002-\mathrm{X}$

NEW YORK STATE ENERGY RES AUTH

Albany, NY

058500-0102-6

NEW YORK STATE OF

Altorney Gen / Envir Protect

Albany, NY

Energy Office

Albany, NY

Energy Research \& Dev Auth

Albany, NY

Health \& Research Dept of

Buffalo, NY

Heallh Dept.

Albany, NY

Buffalo, NY

$275500-0101-4$

284520-0001-4

$275190-0101-6$

$058550-060]-8$

$058550-0701-4$

$058550-2302-8$

$058550-0304-9$

$058550-0302.7$

Housing \& Commun Renewal Div Albany, NY

$058550-220]-3$

NEW YORK STATE UNIV RES FDN

Old Westbury, NY

NEW YORK STATE UNIVERSITY OF

Albany, NY

Binghamoton, NY

NEW YORK TIMES

Parsippany, NJ

NEW YORK UNIV MEDICAL CENTER New York, NY

$039260-0012-0$

$058560-0001-3$

058560-0002-1

275510-0001-1

NEWACT

Republic, WA

$058580-00000$

NEWARK WAYNE CMTY HOSPITAL

Newark, NI

NEWCOMB ANDERSON ASSOC

Anchorage, AK

Ketchikan, AK

La Jolla, CA

Las Vegas, NV

Moscow, ID

Pacific Grove, CA

Pago Pago, Australia

Photnix AZ

Riverside, CA

San Francjsco, CA

Saratoga, WY
$274540-0001-0$

$275200-0001-X$

245180-0007-2

245180-0005-6

$245180-0011-0$

245180-0004-8

245180-0003-X

$245180-0013-7$

$245180-0012-9$

$245180-0010-2$

$245180-0002-1$

245180-0001-3

245180-0006-4
Awardee

Identification

code

Tucson, AZ

$245180-0008-15$

Whiskeytown, CA

245180-0009-9

NEWMARK INTERNATIONAL INC

Beflville, TX

$290710-0001-x$

NEWTON SCIENTIFIC INC

Cambridge, MA

NEXL

Pçabody, MA

$244920-0002-\mathrm{X}$

NEZPERCE \ONT SCH DIST \#302

Nezperce, ID

$287030-0001-\mathrm{X}$

NFT INC

Albuquerque, NM

NI-LECTRA CORP

Daylon, $\mathrm{OH}$

$283630-0001-9$

NICHOLS \& ASSOC

Austin, TX

$227160-0002-6$

$275210-0001-3$

NICOLET COMPUTER GRAPHICS DIV

Washington, DC

NIELSEN ENGINEERING \& RESEARCH

Mountain View, CA

$059030-0001-0$

NINE FORTY EIGHT INC

Baltimore, MD

$288360-0001 \cdot 0$

NISE EAST

North Charleston, SC

NOBLESTAR SYSTEMS CORP

Falls Chwech, VA

NOELEYE DOCUMENTARIES

San Francisco, CA

$243180-0001-X$

290650-0001-1

NORAN INSTRUMENTS

Middleton, WI

NORANDA ALUMINUM INC

New Madrid, MO

$286970 \cdot 0001.7$

$270560-0001-0$

$270570-0001-4$

NORCO COMPUTER SYSTEMS INC

Cleveland, $\mathrm{OH}$

$287810-0001-4$

NORESCO

Framingham, MA

NORFOLK STATE COLLEGE

Norfolk, VA

$288820-0001-X$

NORFOX SOFTWARE FNC

Lynnwood, WA

NORQUAY CONSTRUCTION INC

Tempe, AZ

NORTECH INC

Antigo, WI

NORTEL INC

Springfield, MO

$275250-0001.8$

264520-000I-2

246130-0001-7

$275290-0001-2$

NORTH AMER ELEC RELLABLITY CNL

Prisceton, Ni

$293120-0001-2$

NORTH AMERICAN ACCESS TECH INC

Hawthome, NY

290310-0001-9

NORTH AMERICAN PHILLIPS CO

Briarcliff Manor, NY

$275300 \cdot 0001-2$

NORTH AMERICAN REG TECH CENTER

Clinton, IL

284950.0001 .6

NORTH ARLINGTON BOROUGH OF

North Arlington, NJ

$284240-000 \mathrm{I}-3$ 
Awardee Identification

NORTH BONNEVLLE CITY OF

Planning Divition

North Bonneville, WA

NORTH CAROLINA A \& T STATE $U$

Greensboro, NC

NORTH CAROLNA ELEC MEM CORP

Raleigh, NC

NORTH CAROLINA STATE OF

Adnin Dept

Raleigh, NC

Commerce Dept of

Raleigh, NC

Energy Comm Dept of

Raleigh, NC

Energy Div

Rakeigh, NC

NORTH CAROLINA STATE UNIV

Raleigh, NC

Forestry Dept of

Rateigh, NC

NORTH CAROLINA UNIVERSITY OF

Chapel Hill,

Pembroke, NC

NORTH COAST CRYSTAL INC

Cleveland Heights, OH

NORTH COAST ELECTRIC CO Anasco, PR

St Catherínes, Canada

NORTH COUNTRY PEOPLE'S COOP

Colville, WA

NORTH DAKOTA NATURAL RES

Bismarck, ND

NORTH DAKOTA REAP

Bismarck, ND

NORTH DAKOTA STATE OF

Intergovernment Assist Off of Bísmarck, ND

NORTH GEM SCHOOL DIST \#149 Bancroft, ID

NORTH GREENVTLLE COLLEGE Trgerville, SC

NORTH PEMISCOT CTY R-I SD

Wardell, MO

NORTH PLATTE hOSPITAL CORP

Great Plains Reg Med Ctr North Platte, NE

NORTHBY JAN A

West Kingston, RI

NORTHCOAST CTR POLICY

Arcata, CA

NORTHEAST BY NORTHWEST Alna, ME

NORTHEAST CAKRY

Hallowetl, ME

NORTHEAST ENERGY SERVICES

Framinghlam, MA

NORTHEAST REG CANCER INST

Scranton, PA

287300-0001-7

$059460-0102-7$

$059460-0702-5$

059460-0902-8

$059460-0302-x$

059470.0001 .6

$059470.2101-3$

OF

059490-0009-9

$059490-0008-0$

$289760-0001-3$

059590-0007-5

059590-00008-3

275330-0001-3

275340-0001-7

275350-0001-0

$059660-0301-7$

$266800-000\}-7$

$059770-0001-4$

$282880-0001-0$

$275360-0101-0$

275370-0001-8

$275380-0001-1$

$286390-0001-8$

$275390-0001-5$

$291890-0001-3$
Awardee

Jdentification

conle

NORTHEAST SOLAR ENERGY CENTER

Boston, MA

275400-0001-5

NORTHEAST SUSTAINABLE ENERGY

Greenfield, MA

$285020-0001-2$

NORTHERN CA SOLAR ENERGY

Mountsin View, CA

$275410-0001-9$

NORTHERN CALIFORNIA POWER AGEN

Steom Fleld

Middletown, CA

$180820-0102 \cdot \mathrm{X}$
NORTHERN ESSEX COMMUNITY COLL

Administrative Setvices

Havertill, MA

200200-0101-0

NORTHERN HOMES OE PENNA INC

Chambersburg, PA

275420-0001-2

NORTHERN ILLINOIS UNIVERSITY

Chemistry \& Biochemistry

Dekalb, IL

060250-0403.X

NORTHERN IOWA UNTVERSITY OF

Af-Based Research

Waverly, IA

$105510-0202-6$

NORTHERN MARIANA IS CMNWLTH OP

Emergency Management Office

Saipan, MP

159860-0701-8

Energy Office

Saipan, MP

159860-0101-X

Publtc Works Dept of /Eugg Div

Sajpan, MP

$159860-0601-1$

NORTHERN MUCHGAN HOSPITALS

Petoskey, MI

$282890-0001-4$

NORTHERN NEF INC

Richland, WA

239190-0002-1

NORTHERN NEW MEXICO COMM COL

E] Rito, NM

060330-0001-3

Espanola, NM

$060330-0002-1$

NORTHERN RESEARCH $\&$ ENG CORP

Cambridge, MA

$060350-0002.9$

NORTHERN TELECOM LTD

Alpharetta, GA

269570-0002-8

Burnaby, Comoros

269570-0001-X

NORTHERN UNIVERSISY

Sponsored Proj Adm

Bvanston, IL

275430-0)101-2

NORTHLAND SIDING \& WINDOWS

Bismarck, ND

$257200-0002-\mathrm{X}$

NORTHROP RES \& TECH CENTER

Palos Verdes Peninsula, CA

275440-0001-X

NORTHRUP \& ASSOC

Holidaysburg, PA

$275460-0001-7$

NORTHSTAR COMPANIES INC

Indianapolis, IN

$285880-0001-6$

NORTHWEST ALUMINUM TECHNOLOGIE

The Dalles, OR

NORTHWEST COMMUNITY COLLEGE

Rangely, $\mathrm{CO}$

$060590-0002-9$

NORTHWEST GEOTHERMAL CORP

Lakeview, OR

$116120-0001-0$

Pordand, OR

$116120-0002-9$ 
Awardee $\begin{gathered}\text { Identífication } \\ \text { code }\end{gathered}$

NORTHWEST MISSISSIPPI JR COLL

Senarobia, MS

$060660-0001-2$

NORTHWEST PRECISION AG

Blackfool, ID

292540-0001-9

NORTHWEST RURAL PUB POWER DIST

Hay Springs, NE

290530-0001-1

NORTON CO

Chemical Process Products Akton, OH

NORWEGLAN SEISMIC ARRAY

Kjeller, Norway

NOTRE DAME UNIVERSITY OF

Graduate School

South Bend, IN

NUCLEAR ASSURANCE CORP

Aktau, Kazakhstan

Atlanta, GA

$275470 \cdot 0101-7$

$291430-0001-0$

$060900-0701-3$

060950-0003-3

060950-0001-7

NUCLEAR DATA INC

Woodfield Mall. IL

060960-0004-5

NUCLEAR ENERGY AGENCY

Issy-Les-Moulineaux, France

060970-0002-2

NUCLEAR ENERGY SERVICES INC Danbury, CT

$060980-0002-6$

NUCLEAR MATLS AND EQP CORP Apollo, PA 275480-(0001-4

NUCLEAR MEDICINE SOCIETY OF

Radiopharm Sci Council

New York, NY

076040-0101-7

NUCLEAR OPERATION INSTITUTE OF Atlanta, GA 228800-0001-7

NUCLEAR POWER INSTTTUTE OF Atlanta, GA

$169890-0001-5$

NUCLEAR REGULATORY COMMISSION

Rockuille, MD

NUTTLE DAVID A

Ralejgh, NC

061010-0003-6

NW ENERGY

$275490-0001-8$

Steattle, WA

NWT INC

Salt Lake Gity, UT

NYE COUNTY NEVADA

Nuclear Repository Program

Pahrump. NV

NYE COUNTY OF

Round Mountain, NV

NYFM INC

Latham, NY

286240-0001-7

$291080-0001-8$

$257170-0102-9$

180950-0002-7

$287960-0001-5$

o

O M OFFICE SUPPLY CO

Mechanicsbutg. PA

$288870-0001-8$

O'HARA JAMES C

Corpus Christi, TX

270430-0001-7

OAKLAND BOARD OF EDUCATION

Dakland, CA

$06120010001-1$

OAO CORP

Manvigement Advisory Serv Greenbeht, MD

$061300-0103-7$
Awardee

Identification

code

OASIS PETRO ENERGY CORP

Culver Cily, CA

$275520-0001-5$

OBERBRORCKLING ELMER \& C MOORE

New Vienna, IA

$266070-0001-0$

OBLON FISHER SPIVAL MACL

Arlington, VA

275530-0001-9

OCCIDENTAL COLLEGE

Las Angeles, CA

OCCIDENTAL OIL SHALE INC

Bakersfield, CA

OCCIDENTAL RESEARCH CORP

La Verne, CA

$286870-0001-4$

116160-0003-1

OCCUPATIONAL MED INSTITUTE OF

Edinburgh, United Kingdom $\quad 040070-0001-8$

OCCUSAFE

Wilmette, IL

$061330-0002-3$

OCE OFFICE SYSTEMS

San Francisco, CA

254690-000i-X

OCE USA INC

Chicago, IL

$196350-0002-2$

OCEANIC SOCIETY

Stamfotd, CT

$275540-0001-2$

OCHS GREG

Austin, TX

OCTROOIDUREAU VRIESENDORP

The Hague, Netherlands

$268500-0001-2$

ODIN ASSOC

Cambridge, IA

OFF POLL PREY COMPL ASSSIST

Hartisburg, PA

$061420-0001-4$

$275550-0001-6$

OFFICE AUTOMATION TECHNOLOGIES

Lakewood, $C O$

$288550-0001-2$

OFFICE SOLUTIONS INC

Alexandria, VA

OGDEN CITY SCHOOL DIST

Ogden, UT

$2755700001-3$

275580-0001-7

OGDEN POWER CORP

Mammoth Pacific Lp

Mammoth Lakes, CA

OGLE PETROLEUM INCTS PC

Santa Barbara, CA

OHIO AGRI R \& D CENTER

Wooster, $O H$

OHIO STATE OF

Energy Dept of

Columbus, $\mathrm{OH}$

Energy Efficiency Office of

Coluntous, OH

OHIO VALLEY ELECTRIC CORP

Piketon, $\mathrm{OH}$

OHMART CORP

Cincinnati, $\mathrm{OH}$

OIL CHEM \& ATOMIC WORKERS INTL

Lakewood, CO

OIL WELL AUTOMATION INC

Seneca, PA

OKLAHOMA ELECTRIC COOP

Norman, $O K$
$288100-0101-\mathrm{X}$

$275590-0001-0$

$061520-0001-7$

061570-1901-8

$061570-2001-6$

061620-0001-X

275600-0001-0

260220-0001-7

280280-0001-0

$275610-0001-4$ 


\section{Awardee}

OKLAHOMA STATE OF

Commerce Dept of

Oklahoma City, OK

Community Aftairs \& Dev

Okłahoma City, OK

Corp Commlssion

OkJahoma City, OK

Enersy Dept of

Oklahoma City, OK

Tourism Department Oklahoma City, OK

OKLAHOMA STATE UNIVERSITY

Entyineering College of

Stillwater, $O K$

OKLAHOMA UNIVERSITY OF

Civil Eng \& Env Science

Norman, $O K$

Reseserth Admin Office of

Norman, OK

OLAVSON L G

Sal Lake Cìty, UT

OLSON ASSOC

Lincoln, NE

OLSON HOME PRODUCTS

Catonswille, MD

OLSON WAYNE $R$

Catonswille, MD

OLYMPIC ENGINEERING CORP

Seattle, WA

OMF CONTRACTORS INC

Beitsville, MD

OMNI CABLE CORP

West Chester, PA

ON LOCATION INC

Dunn Loring, VA

ONA JUNIOR HEGH SCHOOL Ona, WV

ONEIDA COUNTY SCHOOL DIST Stone, iD

ONSITE ENERGY CORP

Carlsbad, CA

ONTARIO HYDRO/AECL

Nuclear Saudies \& Safety Dept

Toronto Omtario, Canads

OPEN PLAN SYSTEMS INC

Fairfax, VA

OPERATING ENGRS INTL UNION O

Noth Las Vegas, NV

OPERATIONAL TECHNOLOGJES

San Antonio, TX

OPHFR CORP

Lakewood, $\mathrm{CO}$

OPHHR TOWN OF

Ophir, CO 28077
OPTICAL COATING LABORATORY INC

Industry, $C A$

OPTICAL DATA SYSTEMS

Vientha, VA
Identification eicde

$061750-0602-X$

$061750-1102-3$

061750-0102-8

$061750-0902-9$

$061750-1002-7$

$061770-1401-1$

061780-25(3)-7

$061780-2401-0$

275620-0001-8

275660-0001-2

$275650-0001-9$

$281900-10001-2$

275670-(ด)ก1-6

$256480,0002-2$

293150-000]-3

293140-0001-X

$275690 \cdot 0001-3$

$\$ 351$

264840-0001-8

$265280-0001-8$

$275700-0101-\mathrm{X}$

$288090-0001.3$

254070-0003.2

CORP

$286080-0001-6$

$062120 \cdot(1003.4$

280770-0001-0

$248650-0002-3$

264770-0001-6
Awardee

Identification

code

OPTICAL SOCIETY OF AMERICA

Erecutive Orifle

Washington, $\mathrm{DC}$

$137020-0101-8$

OPTION PROGRAM NEW CASTLE CTE

School District Area III

Newark, DE

$275710-0101-3$

OPTOMEC DESIGN CO

Albuxirerque, NM

$283200-0001-7$

ORACLE CORP

Belmont, CA

$116300-0008-6$

Reston, VA

[ ]6300-00]0-8

San Francisco, CA

$16300-0009-4$

ORAL ROBERTS UNIVERSITY

Chemistry Dept

Tulse, OK

ORANGE TECHNOLOGIES INC

Gaithersburg, MD

ORBITAL SCIENCES CORP

Germantown, MD

$062200-0301-2$

$286090-0001-x$

ORD

Nahant, MA

$292610-0001-0$

ORE-IDA FOODS INC

275720-0001-0

Boișe, ID

$275730-0001-4$

OREGON APPROPRIATE TECH INC

Eugene, OR

275740-00001-8

OREGON COLLEGE OF EDUCATION

Physical Science

Monmouth, OR

OREGON COOPERATIVE FISHERTES

Corvallis, OR

$062270-0101-5$

DREGON \$TATE OF

Economic Development Dept

Salem, OR

275750-0001-1

Fish \& Wldite Dept of

Corvallis, $O R$

Enterpise, OR

OREGON STATE UNIVERSITY

Air Resources

Corvallis, OR

Civil Environmental Constr

Corvallis, OR

Forest Science Dept of

Corvallis, OR

ORENSTEIN MEIR

Tel-Aviv, Iceland

ORG DEVELOPMENT SYS

Washington, DC

$062320-1602-5$

062320-0804-9

$062320-0809-\mathrm{X}$

ORKAND CORP

Hyattsvilke, MD

Silver Spring, MD

ORLANDO UTIL COMAISSION

Orlando, FL.

062330-3101-X

062330-3301-2

$062330-3201-6$

$134650-0002-1$

$275760-0001-5$

$062370-0004-6$

062370-0001-1

OROFINO JOINT SCHOOL DIST \#171

Orofino, ID

$275780.0001-2$

ORSHANSKY TRANSMISSION

San Diego, CA

283560-0001-7

OSAGE CITY CITY OF

Orage City, KS

$275790-0001-6$

$106360-0001-x$ 

Awardes
Identification
code

OSAGE TRIBAL COUNCll

Pawhuska, OK

$284910-0001-1$

OSBORN CHARLES

Sparks, NV

OSBORN DANIEL C

Athens, AL

OSBORN/HODGES/ROBERTS/W

Bryan, TX

OSTERHOUDT II M GLENN

Arlington, TX

OSTGREN ASSOC INC

San Francisco, CA

OTAWA TORU

Landscape Arch Dept

Muncie, IN

OTHMOR MEIER

La Porte City, IA

OTISCA INDUSTRIES LTD

Syracuse, NY

OTT DR KARL 0

Lafayette, IN

OTY WATER ENGINEERS INC

Bellevue, WA

Redding, CA

OTTAWA UNIVERSITY OF

Ottawa, Conada

OUR LADY OF MT CARMEL CHURCH

New York, NY

OUR LADY OF PERPETUAL HELP

New York, NY

OUR LADY OF THE SNOWS SCHOOL

Eugene, MO

OURAY CITY OF

Ouray, $C O$

OUIay, CO
OVERHEAD DOOR CO OF ABERDEEN
Aberdeen, SD

275840-0101-7

275930-0001-X

062540-0001-6

275940-0001-3

$062560-0002-1$

$062560-00001-3$

243890-0002-0

062720-0003-0

$108070-0002-7$

OVONIC BATTERY CO

Troy, MI

OWEN \& MAYES CONST ENG

Springfield, VA

OWEN MURPHY PRODUCTIONS

Westpor1, $\mathrm{CT}$

OWENS ILLINOIS INC

Tọledo, $\mathrm{OH}$

OWENS INIMIS INC

Toledo, OH

OXFORD SCHOOL DEPT

Oxford, MA

$282900-000 \mathrm{i}-4$

275950-0001-7

275960-0001-0

289980-0001-6

$275970-0001-4$

275980-000]-8

062990-0001-5

275990-000]-1

276010-0001-X

\section{$\mathbf{P}$}

P G \& E ENERGY TRADING CORP Houston, TX

286940-00001-6

P/R WIND TANKERS Oslo I, Noway

PA GRADE CRUde OIL ASSN Bradford, PA

063080-0003-5

PACE CO CON $276020-6001-3$ Houston, TX
Awardee

Identification

code

PACE UNFVERSITY

White Plains, NY

PACIFIC ADVANCED TECHNOLOGY

Santa Ynez, CA

PACIFIC ECONOMIC COOPERATION

Washington, DC

286770-0001-1

PACIFIC ENERGY SYSTEMS INC

Pordand, OR

$285560-0001 \cdot 0$

PACIFIC ENVIRON SVCS INC Santa Monica, CA

PACIFIC Gas a ELECTRIC CO

Dakland. CA

$063130-0003-x$

Geysers Power Plant

Healdsburg, CA

$063140-0008-4$

PACIFIC HEALTH RESEARCH INST

Honolulu, HI

PACIFIC NORTHWEST LABORATORY

Richlatid, WA

276120-0001-6

PACIFIC REFINING

Houston, TX

$276130-0001-X$

PACIFIC RESOURCES INC

Honolulu, $\mathrm{HI}$

PACIFIC SIERRA RESEARCH CORP

Santa Monica, CA

PACIFIC SUN INC

Menlo Park, CA

$063140-0409-8$

PACIFIC TRANS SUPPLY CO

San Francisco, CA

PACIFIC WESTERN TECHNOLOGIES

Albuquerque, NM

Page NORMAN L Amherst, MA

PAGENET OF NEW MEXICO

Albuquerque, NM

$063220-000 \mathrm{~L}-2$

$165160-0002-6$

PAHRUMP NEVADA

Pahrump, NV

PAKTANK NEDERLAND B $\mathrm{V}$

Rotterdam, Netherlands

PALAU REPUBLIC OF

Energy Office

Koror, PW

276]40-0001-3

PALMER MARK E

Eugene, OR

PALMER PUBLIC LIBRARY

Palmer, AK

$276150-0001-7$

224070-0002-1

275260-0001-1

275890-0004-9

$292710-0001-3$

$063370 \cdot 0001-3$

PALMOUR GROUP

Livingston. TX

PAMELA HORRELL \& ASSOC INC Pittsburgh, PA

$167360.0201 \cdot X$

$273060-0001-2$

276170-0001-4

$276050-0001 \cdot 4$

PAN ABODE INC

Benton City, WA

248610-0003-3

$276180-0001-8$

PANASONIC BROADCAST \& TV Albuquerque, NM

$283430-0001-3$

PANASONIC INDUSTRLAL CO

Secaucus, NJ

292450-000]-X

PAPER CHEMISTRY ENSTITUTE OF Appleton, WI

040080-0001-1 


$$
\text { Awardee } \quad \begin{gathered}
\text { Identification } \\
\text { code }
\end{gathered}
$$

Chemical Sciences Div

Appleton, WI

$040080-0101-8$

Paper Materials \& Systs Div

Appleton, WI

$040080-0201-4$

PAPEK SCIENCE \& TECH INST OF

Tiber \& Paper Physics Div

Atlanta, GA

$203800-0501-8$

PARADIGM GENETICS INC

Durham, NC

$290250-0001-0$

PARALLAX INC

Golden, $\mathrm{CO}$

$242420-0004-9$

PARKDALE BUILDING MAINTENANCE Blacksburg, VA

$276190-0001-1$

PARKER CONNIE \& ROBEKT

Pavillion. WY

PARMA SCHOOL DIST $\$ 137$

Panma, ID

$276200-0001-1$

PARSONS IN Reading, PA

$259270-(00) 1-0$

ARSONS POW

Reading, $P A$

PARSONS-BRINCKERHOFF

Harahan, LA

Houston, TX

New Orleans, LA

New York, NY

PARSONS-BRINCKERHOFF ENERGY

Denyer, CO

PARSONS-BRINCKERHOFF-QUADE

Giolden, $C O$

PARTNERS ANDRA

Cedar Rapids, IA

PARTNERSHIP INC

Lakewood, $\mathrm{CO}$

PASADENA CITY COLLEGE

Pasadena, CA

PASADENA CITY OF

Pasadena, CA

PASCO COUNTY SCHOOL BOARI

Land $\theta^{\prime}$ Lakes, FL

Pasco, FL

\section{L}

ASCOR PACHFIC AIR SWTCH CORP

Forest Grove, OR

PASSAMAQUODDY TRIBE

Themaston: ME

Council

Eastpor, ME

PASSIVE SOLAR ALTERNATIVES

Rapid City, SD

PAT HARRISON WATERWAY DISTRICT

Hattiesburg, MS

PATHFINDERS INC

Cherry Hill, NJ

PATTERSON A O

Macon, GA

226610-0001-1

$276210-0001-5$

292530-0001-5

$130820-0001-0$

$150000-0001-3$

063790-0003-8

063790-0004-6

203500-0001-8

$139170-0003-2$

139170-0204-3

PATTERSON GENE Springfeld, MO
Awrardee

Identification code

PAUL C RIZZO ASSOC INC

$$
\text { Monroeville, PA }
$$

$285570-0001-4$

PAUL REED CONSTR \& SUPPLY

Momili, NE

$287700-0001-8$

PAYNE RICHARD C

Kents Store, VA

276290-0001-4

PB KBB INC

Harahan, LA

PBNA ARCHITECTS INC

Kansas City, MO

166320-0002-9

PC \& J'S

Bruceton Milts, WV

PCB DISPOSAL SYSTEMS INC

Little Rock, AR

PEABODY COAL CO

Lexingon, MA

PEABODY PROCESS SYSTEMS INC

Stamford, CT

PEAK OIL CO

Tampa, FL

PEARL B LARSEN ELEMENTARY SCH

Charlotte Amalie, VI

$276300-0001-4$

285040-0001-X

$120830-0003-x$

276310-0001-8

$276320-0001-1$

$276340-0001-9$

PEARSON DERTDDER \& JOHNSON INC

Lakewood, $\mathrm{CO}$

PEAT MARWICK MTTCHELL \& CO

Fernald, OH

Washington, DC

PECKWORTH RALPH H

Charlotec, NC

PECO ENERGY CO

Gas Services Group

Philadelpbia, PA

PELGER EDWARD

Philadelphia, PA

PELICAN PUBLIC LIBRARY

Pelican, AK

PELLISSIPPI ST TECH CMTY COLL.

Knoxville, TN

Oak Ridge, TN

PEM TECHNOLOGIES

Bethesda, MD

PENN GEOLOGICAL SURVEY

Envir Res Dept

Harrisburg, PA

PENNSBURY SCHOOL DISTRICT

Levitown, PA

290980-0001-0

064300-0005-7

064100-0006-5

$2763600001-6$

$283370-0101-1$

$276370-0001-\mathrm{X}$

$276380-0001-3$

$240900-0001-7$

$2409(02-0002-5$

$284020-00001-0$

PENNSYLVANIA COLL OF TEX \& SC

Philadelphia, PA

$276390-0101-3$

$064290-0002-8$

PENNSYLVANLA COMMONWEALTH OF

Comm \& Econ Dev Dept of

Hamisbung, PA

064360-1401-2

Governor's Energy Coumcil

Harrisburg. PA

$064360-0401-7$

Public Utility

Hartisburg, PA

Visually Handicapped

Pittsburgh, PA

$064360-1301-6$

$064360-0603-6$ 

Awardee
Identification
code

PENNSYLVANIA STATE UNIVERSITY

Biophysics Dept

University Park, PA

$064370-3102-6$

PENNSYLVANLA UNIVERSITY OF

Surpery Hdsr

Philadelphia, PA

$064380-2201-2$

PENNWALT

King of Prussia, PA

PENNZOIL CO

Exploration \& Production

Parksburg, WV

PENOBSCOT INDLAN NATION

Indian Island, ME

PENQUOS CAP INC

Bangor, ME

PENTA INTERNATIONAL INC Washington, DC

PENTA POST INC

Tutke, ID

PEOPLES NATURAL GAS CO

Pitsburgh, PA

PEOPLESOFT INC

Bethesda, MD

Chicago, IL

Richland, WA

PERDUE ROBERT W

Washington, DC

PERKNN ELMER CORP

Chicago, JL

$276410-0001-0$

$064390-0102-7$

$276420-0001-4$

$276430-0001-8$

$276440-0001-1$

276450-0001-5

276460-0001-9

248520-0002-6

248520-0004-2

$248520-0003-4$

$276470-0001-2$

$064520-0012-8$

PERSONNEL MANAGEMENT OFFICE OF

Charlottesville, VA

Federal Executive Institule

Charlottesville, VA

064630-0013-2

Golden, $\mathrm{CO}$

PET INFO CORP

Dallas, TX

PETER F LOFTUS CORP

Bruceton, PA

Pitssbugh, PA

PETER KIEWTT SONS CO Concord, CA

$064630-1313.7$

$064630-1311-0$

$276490-0001-\mathrm{X}$

$064680-0003-3$

064680-0002-5

$276510-0001-3$

PETER PALICA ATTORNEY AT LAW

Marshtield, MA

$286360-000 \mathrm{~J}-7$

PETERSBURG CITY OF

Mnnicipal Power \& Light

Petersbutg, AK

$064700-0101.7$

Public Library

Petersburg, AK

$064700-0201-3$

PETERSON CO

Baltimore, MD

293]70-0001-0

PETERSON CONSTRUCTION CO INC

Grand Forks, ND

$109620-0002-7$

Miamisburg, OH

$109620-0001-9$

PETERSON RICHARD C

Easıtham, MA

$277270-0001-9$

PETERSON ROGER JOHN Cheyenne, WY
Awardee

Identification code

PETRO LEWIS CORP

Denver, $\mathrm{CO}$

PETROLEUM ENGINEERING

Tulsa, OK

PETROLEUM INFO DWIGHTS LLC

Richardson. TX

PETROLEUM INSTITUTE OF

London, United Kingdows

PETROLEUM LABORATORIES INC

Lafayette, LA

$276530-0001-0$

$276540-0001-4$

$287720-0001-5$

174700-0001-1

PETROLEUM TECH CORP

Redmond, WA

276550-0001-8

ETROLEUM TECHNOLOGY TRANSFER

Washington, $D C$

PHARCHEM LABORATORIES INC Menlo Park, CA

PHELPS PACKTNG RUBBER CO

Baltimore, MD

276560-0001-1

$287990-0001-6$

$239660-0001-6$

PHILIPS ELECTRONIC INSTRUMENTS

Fremont, $\mathrm{CA}$

Hillsboro, OR

0.0006.X

PHILLIPS CITY OF

City Schools

Phillips, WI

$276630-0101 \cdot x$

PHILLIPS CLYDE G

Rehoboth Beach, DE

PHOENIX ELECTRIC CORP

Cation, MA

$276600-0001-2$

PHOTON IMAGING INC

Northridge, CA

$276610-0001-6$

PHOTOWATT INTERNATIONAL Tempe, AZ

PHYSICAL ELECTRONICS INC

Ellicolt City, MD

PHYSICAL MIED \& REHAB INST OF Loujsvilte, $\mathrm{KY}$

PHYSITRON INC

Huntsuile, AL

$283210-0001-0$

276620-0001-X

285210.0001 .4

040090-000t-5

284420-0001-1

PICKARD LOWE \& GARRICK INC Washinglont, DC

PICTURETEL CORP

Andover, MA

$154500-0002-2$

PIEDMONT TECHNICAL COLlEGE

Greenwood, SC

251730-0003-1

PIELKENROAD SEPARATOR CO

Houston, TX

PIERCE MANUFACTURING INC

Applelon, Wl

PIETRI JOSE M

Yauco, PR

PIHAF SCHMIIT WESTERDAHL

Porlland, $O R$

065190-00001-9

$276640-0001-7$

$246040-0001-8$

$276650-0001 \cdot 0$

PINEHURST.KINGSTON FREE LIB

Pinehurst, ID

$276660-0001-4$

PIONEER NATURAL RESOURCES USA Irving, TX

PIRANHA PRODUCTS CO

San Diego, CA

276680-0001-1 


\section{Awardec \\ Identification \\ code}

PIRELLI CABLE CORP

Lexington, SC

PIRELLI CABLE SYSTEMS

New York, NY

PITKIN LUCIUS INC

Grand Junction, $C O$

PITNEY BOWES INC

Annandale, VA

Golden, $\mathrm{CO}$

PITTSBURGH PARTNERSHIP INC

Atlanta, $G_{A}$

PITTSBURGH UNIVERSITY OF

History \& Lanzuage NDMK

Pittsburgh, PA

Sponsored Programs Offle of

Pittsburgh, PA

PITTSTON CO

Greenwich, CT

PIUS X HIGH SCHOOL

Lincoln, NE

PJR CONSTRUCTION CORP Brooklyn, NY

PLAINS ELECTRIC $\&$ \& T COOP INC Albuquerque, NM

PLANERY

Austin, TX

PLANET EARTH SCJENCE INC

Sarta Barbara, CA

PLANNING \& MANAGEMENT ASSOC Washington, DC

$151200-0003.7$

$276690-0001-5$

$276700-0001-5$

$065440-0012-4$

$065440-0011-6$

$286520-000) 1-8$

$065490-2501 \cdot X$

$065490-2601-6$

$276710-0001-9$

$282810-0(x) 1-5$

$276720-0001-2$

$180850-0001-6$

$276740-0001-X$

PLANNING DEVELOP \& MANAGEMENT Chico, CA

PLASMAQUEST INC

Richardson, TX

PLASTRONIC INC Troy, $\mathrm{OH}$

PLATINUM TECH

Vjenna, VA

PLATT RIVER INDUSTRIES

Denver, $C O$

$276760-000 t-7$

$283960-0001-8$

$284320-0001 \cdot 9$

$259590-0002-4$

PLATTEVILLE FIRE PROTECTION Platleville, CO

PLUG POWER LLC

Latham, NY

$283880-0001-2$

287830-000L-1

285400-0001-6

PLUMMER \& WORLEY JNT SCH D Pluminer, ID

283510-0001-9

PLYMOUTH CITY OF PJymouth, MN

POCAHONTAS COUNTY FREE LIA Green Bank. WV

$276770-0001-0$

POINT REYES BIRD OBSERVATORY

Bolinas, CA

$276300-0001-8$

POLAR CRYOGENICS

Portand, OR

POLICY PLANNING \& EVALUATION

Molean, VA

Reston, VA

$91980-000 \mathrm{~L}-2$

$065910-0001-1$

$065910-0002-\mathrm{X}$

\author{
Awardee
}

Identification code

POLITECH CORP

Washinglon, DC

POLYDYNE

Menlo Park, CA

POLYMATH ASSOC

Pleasanton, CA

POLYSET INC

Manchester, MA

POLYSOLAR INC

Lincolnton, NC

POLYTECHNIC INST OF NEW YORK

Aerodynamics Labor

Brooklyn, NY

Physics Dept

Brooktyo Heights, NY

POPE EVANS \& ROBBENS

Alexandria, VA

New York, NY

PORT AUTHORITY OF NY \& N

New York, $\mathbf{N Y}$

PORT LIONS PUBLIC LIBRARY

Port Lions, AK

PORTLAND CEMENT ASSOC

Skokit, IL

Research \& Development

Houghton, MI

Skokie, IL

PORTLAND CITY OF

Job Corp Center

Portland, OR

POSEDON RESEARCH

Los Angeles, CA

POST FALLS SCHOOLS DIST \#273

Post Falls, ID

POTEAT ROBERT M

Oak Ridge, $\mathrm{TN}$

POTRATZ ROGER F

Lander, WY

POTTER COUNTY

Amarillo, TX

POWDER RIVER ENERGY CORP

Sundance, WY

POWELL ENERGY PROLUCTS

Powell, $\mathrm{OH}$

POWER CONVERSION PRODUCTS INC

Crystal Lake, IL

POWER CONVERSION TECH INC

Harmony, PA

POWER ENGNEERING CORP

Lakewood, $\mathrm{CO}$

POWER ENGNNEERS INC

Loveland, $\mathrm{CO}$

POWER KINETICS INC

Troy, NY

POWER PRODUCTS INC

Sharon Hill, PA

POWER STRUCTURES INC

Houston, TX
276810-0001-1

276820-0001-5

291170-0001-7

276830-0001-9

$065940-0002-0$

065960-0501-1

$065960-0102.4$

066070-0003-7

$066070-0002-9$

276840-0001-2

276870-(1001-3

0662 10-0001-4

$066210-0202-5$

066210-0201-7

066220-0503-6

276880-0001-7

264830-0001-4

2846901-0001-2

277660-0001-6

271190-0001-2

287270-0001-X

$2882 \mathrm{~J} 0 \cdot 0001 \cdot \mathrm{X}$

$120850-000]=0$

$283020-0001-9$

103710-0013-0

259120-0002-8

135200-0001-6

287980-0001-2

$194780-0002-0$ 

Awardee
Identîfication
code

POWERLIGHT CORP

Berkeley, CA

$249070-0001-4$

POZZI MARIO

Via Pioda, Sweden

PRAIRIE ROSE INC

Jamestown, ND

PRATT \& WHITEY AIRCRAFT

Middletown, CT

PRAXAIR INC

Danbury, CT

PRC SYSTEMS SCIENCES

McLean, VA

PRECISION MAGNETIC BEARING SYS

Cohoes, NY

PREMIER ENTERPRISES

Golden, $\mathrm{CO}$

PREMIO COMPUTER INC

Irvine, $\mathrm{CA}$

Orange, $\mathrm{CA}$

PREMISYS COMMUNICATIONS INC

$\begin{array}{ll}\text { E1 Monte, CA } & 286440-0002-0 \\ \text { Fremont. CA } & 286440-0001-2\end{array}$

PRESSET INC

Maricta, GA

PRESTON-CARNEGIE LIBRARY

Presion, ID

$289550-0001-4$

PRIBILOF ISLAND SCHOOL DIST

St Paul, AK

PRICE MICHAEL

Socorro, NM

$066610-0002-3$

$276890-000]-0$

$276900-0001-0$

$252170-0001-5$

276920-0001-8

289230-0001-9

291470-0001-5

$288200-0001-6$ $288200-0002-4$

$276930-0001-1$

]20860-0001-4

$273730-0001-0$

PRICE MICHAEL S

Rohnerı Park, CA

PRICE WATERHOUSE \& CO

Washington, $\mathrm{DC}$

PRICE WATERHOUSE LLP Aslington, VA

PRICEWATERHOUSECOOPERS

Arlington, VA

Fairfax, VA

PRIDE WEED CONTROL INC Montrose, $\mathrm{CO}$

PRIM\&R

Bostọn, MA

PRIME COMPUTER INC Rockville, MD

PRIMUS MARKETING ASSOCIATION Minnetonka, MN

$273770-0001-5$

$066810-0001 \div 0$

287870-0001-6

$291110-0001-5$

$291110-0002-3$

$287880-0001 \cdot \mathrm{X}$

2B7220-000]-]

$066840-0022-4$

PRISM COMPUTATIONAL SCIENCES Madison, WT

PRO TEC ROOFING INC

Watertown, SD

$289130-0001 \cdot 6$

PROCON INC

Contrats and Grants Office of

Des Plaines, IL.

PROCTOR ENGINEERING GROUP

San Rafiel, CA

PRODGERS RICHARD A

Helena, MT

$286110-0001-3$

$067000-0101.3$

$279540-0001-\mathrm{X}$

277250-0001-1
Awardee

Identification

code

PRODUCTION MANAGEMENT CORP

Energy Research Center For

Lexington, $\mathrm{KY}$

$276950-010]-5$

PRODUCTS RES \& CHEMICAL

Burbank, CA

PRODUCTS WF CORP

Garland, TX

$276960-0001-2$

PROFESSIONAL ASSEMBLY SYST SVC

Eagle Bette, SD

$276970-0001-6$

Maurine, SD

245730-0003-8

Newell, SD

245730-0005.4

245730-0004-6

PROFESSIONAL BUS SYS INC

Albuquerque, NM

$283040-0001-6$

PROFESSIONAL SEARCH CONSULTANT

Houston, TX

$291370-0001.2$

PROJECT ASSISTANCE CORP

Richland, WA 264800-0001-3

PROJECT PERFORMANCE CORP

McLean, VA

Sterling, VA

288170-0002-7

$288170-0001-9$

PROJECT SOFTWARE \& DEV INC

Woburn, MA

$2900730-0001-7$

PROJECT SOFTWARE DEVELOPMENT

Reston, VA

$288610-0001-0$

PROTO.POWER MGMT CORP Groton, CT

$067200-0001-2$

PROTON ENERGY SYSTEMS

Rocky Hil, CT

$288010-000] \cdot 4$

PROTRAIN SYSTEMS

Richlath, WA

PROVIDENCE CENTER INC Amold, $M D$

$284470-0001-\mathrm{X}$

PROVIDENCE CORP

Providence, Rl

$277000-0001-8$

PSDI INTL SOFTWARE INC Bedford, MA

$277010-0001-1$

$292810-0001-6$

PUBLIC HEALTH SERVICES

Denver, CO

Golden, $\mathrm{CO}$

PUBLIC PROC RESEARCH FDN

Lexington, $\mathrm{KY}$

PUBLIC SERVICE CO OF COLORADO Denver, $\mathrm{CO}$

PUBLIC SVC CO OF NEW MEXICO

Albuguerque, NM

PNM Euergy Services

A]buquetque, NM

279520-0001-2

279520-0002-0

$277020-0001-5$

$067390-0001-8$

PUEBLO COMMUNITY COLLEGE

Seeds

Pueblo, Co

$280340-0001-9$

$280340-0101-5$

PUEBLO OF ISLETA

$292840-0101 \cdot 3$
Isleta, NM

PURBLO OF LAGUNA Laguna, NM

285380-0001-2

PUEBLO OF POJOAQUE

Enviranmental Dept

Santa Fe, NM
245440-0001-?

$265060-010]-1$ 


$$
\begin{gathered}
\text { Identificatiog } \\
\text { code }
\end{gathered}
$$

PUERTO RICO COMMONWEALTH OF

Natural \& Env Resources Dept

Puerta De Tierta, PR

San Juan, PR

Planning \& Eng Dept

San Juan, PR

PUERTO RICO UNIVERSITY OF Mayaguez, Puerto Rico

San Juan, Pueslo Rico

067490-0805-4

067490-0803-8

$067490-0906-9$

$067500-0006-1$

$067500-0007 \times \mathrm{X}$

PUERTO RICO WATER RES AU

Culebra, PR

277030-0001-9

PUGET SOUND COUNCIL OF GOVT Seattle, WA

PUGET SOUND NAV SHIPYARD

Bremerton, WA

PUGH+ROBERTS ASSOC

Cambridge, MA

PULLMAN SWINDELL Pittsburgh, PA

$277040-0001-2$

PUNA SUGAR CO

Honolulu, HI

PURAQ CO

New Haven, CT

$277050-0001-6$

277060-0001-X

$277070-000 \mathrm{~d}-3$

$277080-0001-7$

$160250-0002-6$

PURCHASE AREA DEVELOPMENT DIST

Mayfield, KY

PURDUE ROBERT $W$

Arlinglon, VA

PURE AIR ON THE LAKE

Allentown, PA

288770-0001-5

277580-0001-0

PURVIN \& GERTZ INC

Dalias, TX

Washington, DC

PUTNAM THOMAS H

Brookings, SD

PX CHARLESTON ENC

Charleston, SC

PYLE NORMAN R Griffin, GA

$230960-0001-8$

$067590-0002-1$

067590-0001-3

267580-0001-X

277090-0001-0

275270-0001-5

PYRAMID LAKE PAIUTE TRIBAL CNC Nixon, NV

288350-0001-7

$\mathbf{Q}$

Q SYSTEMS INC

McLean, VA

$287110-60001-5$

QEI INC

Bedford, MA

Denver, CO

QSS GROUP INC

Lanham, MD

QUAD COUNTY COMMUNITY ACT AGCY

Grand Forks, ND

QUALITY COUNCIL ON ENVIRON

Washington, DC

266360-0001-5

QUALITY INN OF KEY WEST

Miami Beach, Fl,

QUANTUM COMPLIANCE SYSTEMS

Ann Arbor, MI

$277[10-000]-4$

290690-0001-6

$067710-0005-2$

290130-0001-0
Awardee

Identification code

QUEBEC UNIVERSITY OF

Mostreal, Canada

QUEST INTELLIGENCE BUREAU

Boulder City, NV

QUEST MEDIA \& SUPPLIES INC

Sacramento, CA

QUINDAR PRODUCTS LTD

Concord, Comoros

$288220-0001-3$

$266750-0001-2$

265090-0001-6

280480-0001-6

$\mathbf{R}$

R \& $K$ INTERNATIONAL CO

Laguna Hills, CA

R \& M ENTERPRISES

Ridgecrest. CA

R R R LTD

lndependence, MO

R H PRATT ASSOC

Kensington, MD

R H WHELAN CO

ldalia, MO

Neosho, MO

R P PUBLISHING INC

Propane Vehicle Conference Denvet, $\mathrm{CO}$

R SHRIVER ASSOC

Parsippany, NI

RACE INC

Pittsbargh, PA

RADLATION RESEARCH SOCIETY Oak Brook, il

286490-000]-0

275910-0001-2

$287670-0001-0$

$2769\} 0-0001-4$

067940-0008-3

067940-0009-1

RADIOACTIVE ISOLATION CONSORT

Falls Chumch, VA

RADIOTRACER INC

Guilford, CT

RAI-RAPID INC

North Las Vegas, NV

RAMED EARTH DEV CO

Newport, RI

RAMP FREDERICK L

Palikit, Ecuador

RANCHO HIGH SCHOOL

Asmst

North Las Vegas, NV

$290520-0101-4$

$067970-0002-5$

$278260-0001-7$

(6681200004-3

$288840-0001-7$

$28924000001-2$

$237290-0002-0$

$277160-0001-2$

$277180-0001-X$

RANDALL DR TOM M Mapleville, RI

RANDY L HOLLOMAN \& ASSOC Valdosta, GA

RATON TECH Raton, NM

RAY EDWARD D Lawton, $\mathrm{OK}$

RAYBOY E R Margate, FL

RAYTHEON CORP Sudbury, MA Waltham, MA

REAL-TIME ZONE INC Roswell, NM
$265340-0001-6$

269290-0001-9

INC

288790-0001-2

$265940-0001-2$

265590-000I-X

$068660-0005-\mathrm{X}$

068660-0005-8

293070-0001-8
285460-010]-4 
Identification
Awardee

REB RESEARCH CONSULTING CO

East Lansing, MI

Oak Park, MI

RECOM TECHNOLOGIES INC

Campbell, CA

REI CONE RESEARCH INC

Conifer, $\mathrm{CO}$

RED SMMPSON INC Durant, OK

REDCOM LABORATORIES INC Victor $_{x} \mathbf{N Y}$

REFRACTORY COMPOSITES INC Glent Burnie, MD

RETS JOHN C Prescota Valley, AZ

RELIABLE CHEVROLET Springfietd, $\mathrm{MO}$

RENEE GODON \& SONS INC Auburn, CA Foresthill, CA

RENEWABLE ENERGY POLICY PRO Washington, DC

RESEARCH \& DEV SOLUTIONS INC McLean, VA

RESEARCH ELECTRONICS FNTL Cookeville, TN

RESEARCH FND OF THE CITY New York, NY

RESEARCH FOUNDATION OF SUNY Geneșeo, NY

RESEARCH PARTNERSHIP Palo Alto, CA

RESEARCH PLANNING INC Geimantown, MD

RESOLVE INC Washington, DC

RESOURCE \& DESIGN San Francisco, CA

RESOURCE CONSERVATION Renton, WA

RESOURCE MANAGEMENT INTL Sacramento, CA

RESOURCE MG Madison, WI

RESOURCE MGMT INTERNATIONAL Sacramento, CA

RESOURCE PLANNING ASSOC INC Cambridge, MA

RESOURCE TECHNOLOGY CORP State College, PA

RESOURCES FOR THE FUTURE Washington, DC

RETICULAR SYSTEMS INC San Diego, CA

REUAEN H DONNELLEY CORP Oak Brook IL

REUTERS NEWMEDLA INC Reștọn, VA
$233300-0001-5$ $233300-0002-3$

$195260-0003-x$

$292070-0001-6$

292680-0001-6

$280320-0001-1$

289200-0001-8

$2927600001-1$

$29 \pm 000-0001-9$

290480-0002-5

290480-000]-7

290280-000]-]

$271200-0000]-2$

292390-0001-1

291030-0001-X

$286960-000 \mathrm{I}-3$

$283220-0001-4$

$233930-0003-9$

$293270-0001-3$

285840-0001-1

$206350-0002-4$

285310-0001-7

DISON $285420-0001-3$ 275860-00001-8

069510-0001-8

222390-0002-3

$069550-0001-2$

$283230-0301-8$

$264740-0001-5$

$280330-0002-3$
Awardete

Identification cinde

Washitzgton, DC

REYNOLDS LAWRENCE INC Richmond, VA

$280330-000]-5$

REYNOLDS METALS CO

Chester, VA

Shetifild, AL

REYNOLDS SMITH \& HILLS

Jacksonville, FL

REYTECH CORP

Bend, OR

RHINO CONSTRECTION INC

Lower Butrell, PA

277190-0001-3

$069690-0005-2$

$069690-00044$

277200-0001-3

289020-0001-X

215880-0002-8

RHODE ISLAND JUNHOR COLLEGE

Engineering Dept

Warwick, RI

RHODE ISLAND STATE OF

Kingston, RI

$277210-0101-3$

Admin Department of

Providenet, RI

Governor's Energy Office

Providence, RI

$069850-0004-5$

RICCARDO RICHARDS ELEMENTARY

Charlotte Amalie, VI

$069850-0902-6$

RICE UNIVERSTTY

Zoology Department of Houston, TX

RICE WILLLAM MARSH UNIV

Houston, TX

RICHARD B KLAR LAW OFFICE OF

New York, NY

$281760-0001-9$

RICHARD J BIGDA \& ASSOC

Tulsa, OK

RICHARD STOCKTON COLL OF NJ

Pomona, N]

$277300-0001-6$

RICHARD THOMAS G

Fairview, OK

$247100-0001-8$

RICHARDS W H

Silsbee, TX

267540-0001-5

RICHLAND COMMUN COLL INST TECH Decatur, IL

$277370-0001-1$

RICHMOND UNTVERSTTY OF

Richmond, VA

$118670-0001 \cdot 6$

RICOH CORP

San Francisco, CA

$070190-0005-3$

RIMKUS THOMAS W

Huntsville, AR

RIO BLANCO NATURAL GAS

Denver, $\mathrm{CO}$

$267630-0001-4$

RIO DE JANEIRO FEDERAL UNIV OF

Rio De Jarkiro, Brazil 267000-0001-7

RIO GRANDE COMMUNIFY DEV CORP Abuquetqute, NM
$O$ VISTA ENERGY MARKETING Bakersfie]d, CA

$266710-0001-8$

RIRIE JOINT SCHOOL DIST \#252

Ririe, II

231480-0101.X

RISBERG POWER ELECTRONICS INC New Berlin, WI 

Awardee
Identification
code

RISOE NATIONAL LABORATORY

Dk-4000 Roskilde, Denmark

$277420-0001-6$

RITE WAY SERVICES

Albuquerque, $\mathrm{NM}$

131030-0002-2

RITTELMANN ASSOC

Butler, PA

277430-0001-X

RIVER DISTT

St Clair, MI

RIVER RUN FARM

Gilsum, NH

RMC INC

Pueblo, $\mathrm{CO}$

ROANOKE COLLEGE

Salem, VA

ROANOKE INDEPENDENT SOURCT

Salem, VA

ROBBINS GIOLA INC

Alexandria, VA

ROBERT PLETT ASSOC LTD

Washington, DC

ROBERT REILLY \& ASSOC

Northfield, IL

ROBERTS C KENNETH

Houston, TX

ROBERTS NORRIS

Mouni Vernon, NY

ROBERTSON PRECISION INC

Redwood City, CA

ROBINSON I H

Pawleys Island, SC

ROCHESTER UNIVERSITY OF

River Compus

Rochester, NY

ROCKAWAY TOWNSHIP

Rockaway, NJ

ROCKETDYNE

Canoga Park, CA

ROCKLAND COMMUNTTY COLL New York, NY

ROCKLAND SCHOOL DIST \#382

Rockland, ID

ROCKWELL INTERNATIONAL CORP Golden, $\mathrm{CO}$

ROCKY FLATS OFFICE

Albuquerque, NM

ROCKY MOUNTAIN INSTITUTE

Green Development Services

Snowmass, CO

ROCKY MOUNTAIN NATL PARK

Esles Park, CO

ROCKY MTN REG COOP ADMIN SU

Golden, CO

RODEWAY INNS OF AMERICA

Columbus, TX

Dallas, TX

RODRIQUEZ DR PAUL

Garden Ciry. KS
HOSP

$070370-0001-9$

$266840-0001-1$

290790-0001-9

277440-0001-3

$277450-0001-7$

292040-0001-5

277530-0001-2

292380-0001.8

290860-0001-0

275280-000]-9

$2890300001-3$

$277600-0001-4$

070700-1901-7

$285390-0001-6$

$277610-0001-8$

$070810-0003-7$

267020-0001-4

070870-0004-7

284150-0001-4

283860.0101-1

287970-0001-9

$291320-00001-4$

27764i)-0001-9

277640-0002-7

$277650 \cdot 0001-2$
Awardee

Identification

code

ROGER PROTAS ASSOC

Pittsburgh, PA

ROGERS SURVEYING INC PS

Ricbland, WA

ROLLA COMMUNITY HOSPITAL. Rolette, ND

ROLLIN INC

Stroudsburg, PA

ROLLING PLAINS TECH

Swetwaler, TX

ROMINE CONSTRUCTION

Newark, OH

RON WTTHERSPOON INC

Campbell, CA

ROSANDER R T

Young Harris, GA

ROSCOE COMMUNITY CLUG Roscoe, MT

ROSE-HULMAN INST OF TECH

Terre Haute, IN

ROSENAU ENTERPRISE

Wassiburn, ND

ROSENTAL JERRY A

Louisa, VA

ROSS JAMES F

Naperville, IL

ROSS JAMES W

Scottșdake, AZ

ROSS JOSEPH C

Rossville, GA

ROTH BERNHARD G

Livermore, CA

ROTORDYNAMICS-SEAL RESEARCH

North Highlands, CA

$277700-0001-7$

$277720-0001-4$

$277730-0001-8$

$277740-0001-1$

$277750-0001-5$

$284930-0001-9$

283970-0001-1

$277140-0001-5$

277760-0001-9

$277770-0001-2$

$257220-0005-1$

$270660-0001-3$

$270470-0001-1$

$277790-0001-\mathrm{X}$

$271020-10001-4$

ROUSE CONSTRUCTION CO

Oak Ridge. TN

ROWE CONTRACTING SERVICE CO

New Orleans, LA

ROWLEY SCHEN REPROGRAPHICS

Beltsville, MD

ROXPEPE POWERS \& ASSOC

Washington, DC

ROY THOMAS T

Philomath, OR

ROYAL COLLEGE FOR DEFENSE STDS

London, Egypt

$277830-0001-0$

RS INFORMATION SYSTEMS

Mclean, VA

292780-0001-9

RUBEN AUTORIDAD REGULATORIA

Buenos Aires, Argentina

291760-0001-X

RULIFSON ROBERT L

Beltevue, WA

$277490-0001-1$

RUNREUTHER F ASSOC

New York, NY

$277840-0001-4$

RUNSER RICHARD

Wasilla, AK

RUNZHEIMER \& CO

Rochester, WI

$277330-0001-7$

277850-0001-8 

Awartee
Identification code

RUPPNECHT \& PATASHNICK CO Albany, NY

$289050-0001-0$

RURAL ECONOMICS INSTITUTE Woodruff, Wh

RUSSELL LYNN D

Starkville, MS

RUSSELL SAGE FOUNDATION New York, NY

RUSSIAN ACADEMY OF SCIENCES

Physical Chemistry Inst of Moscow: Russian Federation

RUSSIAN UKRAINLAN LEGAL GR Washingcon, $D C$

RUSTY NAIL PIZZA PARLOR Buhl, iD

RW ASSOC INC Tucson, AZ

RYAN HOMES Pittsburgh, PA

RYAN JUNIOR HIGH SCHOOL Fairbanks, AK

RYAN THOMAS C Houslon, TX

RYKERSON DEANE

Worthington, MA

$290270-0101-4$
$280 P$
$288240-0001-0$
$277900-0001-2$
$277910-0001-6$
$277920-0001-x$
$277930-0001-3$
$277940-0001-7$
$277960-0001-4$

\section{$\mathbf{S}$}

S A HOLDITCH ASSOC INC New Otleans, LA

$S$ EDWARDS INC Font Collins, $\mathrm{CO}$

S M STOLLER CORP Idaho Falls, ID

SACRED HEART SCHOOL New York, NY

SADDLECRAFT INC

DBA The Cherokees Cherokes, NC

SAFE ENERGY COMMUNICATION COUN Washington, DC

SAFE ENERGY RESEARCH GROUP New Orleans, LA

SAGE ELECTROCHROMHCS Piscatak'ay, NJ

$265670-0401-5$

$291520-0001-\mathrm{X}$

$071660-0002-3$

$071880-0023-9$

SAI COMIPUTER SERVICE McLean, VA

SAIN ENGINEERING ASSOC Clearwater, FL Gulfport, MS Key West, FL Miami Beach, FL

SALESCO SYSTEMS Phoenix, AZ

SALII LAZARUS E Saipan, $\mathrm{CQ}$

SALK INSTITUTE San Diego, CA

278010-0001-3

244990-0005-X 244990-0002-5 244990-0003-3 244990-0004-1

292460-0001-3

272080-00001-8

$289330-000 \mathrm{l}-\mathrm{I}$
Awardee

Identification code

SALMON LIBRARY ASSOC

Salmon, dD

$278000-0001-7$

SALMON SCHOOL DISTRICT \#291

Salmon, ID

SAM STEWART \& ASSOC INC

St Paul, MN

$283610 \cdot 0001-1$

SAMBOS RESTAURANT

Sanla Barbara, CA

$265850-0001-3$

SAMUEL SPRING

Blairsville, GA

$278030-0001-0$

SAN BERNARDINO CTTY OF San Bemardino, CA

$278040-00014$

SAN BERNARDINO CO San Bernardino, CA

SAN DIEGO STATE COLLEGE San Diego, CA

SAN DIEGO STATE UNIV FDN Regional Energy Office

San Diego, CA

SAN DIEGO UNIVEKSITY OF San Djego, CA

278050-0001-8

SAN FRANCISCO CITY OP

Solid Waste Program

San Francisco, CA

SAN JACLNTO COLLEGE

Lewisetta, VA

CTR

259320-0001-5

$278060-0001-1$

SAN JOAQUIN DELTA COMMUNITY Stockton, CA

SAN JUAN COAL SALES CO INC Newark, NJ

154610-0101.7

293200-0001-8

SANDERS ASSOC INC Kitiery, ME

SANITECH INC Twinsburg, $\mathrm{OH}$

SANSOM VICTOR H Pato Alto, CA

SANTA CLARA SOLAR RES Santa Clara, CA

SANTA CRUZ SOLAR COLLEGE Boulder Creek, CA

$236210-0201 \cdot 4$

$072390-0002-8$

278070-0001-5

$072450-0004-2$

$072590-0002-3$

$072640-0001+x$

$278080-0001-9$

$278090-0001-2$

278100-0001.2

SANTA MONICA COMMUNITY COLLEGE Los Angeles, CA $072750-0002-4$

SAOUGATUCK TOWNSHIP PARK \& REC Douglas, MI

$278110-0004-6$

SAPIENT CATALYTECHS INC Houston, TX

SARASOTA COU $284380-0001-0$ Sarasota, $F$

SATHER CONSTR \& BLDG SUPPLY Granite Falls, MN

SATURN SYSTEMS INC Sunnyralt, CA

SATZLER RON Princeville, IL

SAULT SAINTE MARIE AREA PUBLIC Sault Sainte Marie. MI SAUNDERS CONSTRUCTION CO Santa Fe, NM
072840-0001.5

$286230-0001-3$

278120-0001-X

$278130-01001-3$

278140-0001-7

072930-0002-2 
Awardee $\begin{gathered}\text { Identification } \\ \text { code }\end{gathered}$

SAUNDERS ROBERT S

Olympia, WA

SAUTER SUSAN G

Morgantown, WV

SAVERY WILLIAM C

Philadelphia, PA

SAXENA DR SATISH C Skokic, ll

SBA/SERNA \& CO

New Orleans, LA

SCAA INC

Denver, $C O$

SCALES CONSTRUCTON INC

Bakersfield. CA

SCANLIN DENNIS

Morgantown, WV

SCANLON MARK

Flagstaff, $A Z$

SCENIC AJRLINES INC

Las Vegas, NV

SCHAETZLE W T

Tuscaloosa, AL

SCHEFFER KARL D

Scotia, NY

SCHEPENS EYE RESEARCH INST

Boston, MA

SCHERER ASSOC INC

Mission Viejo, CA

SCHILTZ TONY

Junction City, KS

SCHLEPER M A

Vills Hills, $K Y$

SCHLUMBERGER TECH WELL SVCS

Fairmont, WV

SCHLUSSLER LARRY

Goleta, CA

Willow Creck, CA

SCHMITT \& LESLIE INC

Washington, DC

SCHNATHORST ALVIN C

Huron, SD

SCHNEIDER LIFT TRANGLATOR CORP

Frving, TX

SCHOENHARD FORD

Hurọ, SD

SCHOONOVER JOANN

Seward, AK

SCHOTT OPTICAL GLASS INC

Duryea, PA

SCHWARBER JAMES A

Faibanks, AK.

SCHWEITZER ENGINEERING LAB

Pultman, WA

SCHWEITZER LABS INC

Pullman, WA

SCIENCE \& TECH MOLICX OFFC

Washington, $D C$
$277540-000 !-6$

$280180-0001-8$

$278150-0001-0$

073030-0001-1

$285280-000 \mathrm{~L}-\mathrm{X}$

$278160-0001-4$

$255960-0001-1$

$264290-0001-X$

$273080-0001-X$

$278170-000 t-8$

$281680-0001-3$

276180-0001-1

$213520-0001-6$

2848 10-0000]-9

$280730-000 t-6$

$272680-0001-4$

278190-0001-5

$073160-0002-3$

073160-0003-1

$290840-0001-3$

292640-0001-1

269110-0001-7

290450-0001-6

$270710-0001-8$

$278220-(\mathbf{X}) 01-2$

$270420-0001-3$

$104650-0001 \cdot 0$

$286260-0001-4$

OF

$275560-0002-X$
Awardee

Identification

code

SCIENCE APPLICATIONS INC

Colorado Springs, $C O$

Forr Collins, $\mathrm{CO}$

Golden, CO

Hanna, WY

La Jolla, CA

McLean, VA

Morgantown, WV

Palo Alio, CA

Rock Springs, WY

San Diego, CA.

Man-Computer Systems

Denver, $\mathrm{CO}$

Plasn ra Reseanch Institute

La Jolla, CA

SCIENCE APPLICAMONS NNTL CORP

Aiken, SC

Gaithersburg, MD

Germantown, MD

Vienna, VA

Washingion, DC

Watertown, SD

Technołogy Services Co

San Diego, CA

SCIENTIFIC \& AUS MINICOMIPUTERS

Elberton, GA

SCIENTIFIC ASSOC

Santa Monica, CA

SCIENTIFIC COLUMBUS INC

Dublin, OH

Rochester, NY

SCIENTIFIC CONSULTING GROUP

Bionemediatiou Reseanch Cons

Gaithersburg, MD

SCIENTIFIC ENERGY SYSTEMS

Watertown, MA

SCIENTIFIC SERVICES INC

Austin, TX

Redwood City, CA.

SCIENTIFIC SOFTWARE CORP

Denver, $\mathrm{CO}$

$073650-0022-8$

073650-0020-1

073650-9016-3

073650-0017-1

073650-0006-6

$073650-0007-4$

$073650-0008-2$

$073650-0021-\mathrm{X}$

$073650-0019-8$

073650-0023-6

$073650-1015-0$

$073650-0906-3$

$135470-(0)(1) 7-6$

135470-0019-X

$135470-0013-0$

$135470-0020-3$

I3547(- $-0021-1$

135470-(0)18-1

$135470-0805-0$

098550-0002-1

$073690-0001-\mathrm{X}$

$073700-0006-0$

$073700-0007-9$

SCORE

Medford, MA

SCOTSMAN AUTOMATIC CORP

Barringlon, $\mathbf{L}$.

SCOTT COUNTY SCHOOL DIST \# Austin, IN

$275920-0101-2$

073720-0x001-7

$278350-0002-4$

$278350-0001-6$

$278360-0001-\mathrm{X}$

$278370-0001-3$

$278380-0001-7$

SCOTT LONG CONSTRUCTION CO INC Glendive, MT

SCOTT RJCHARD W

Riverton, WY

SCOTTSBLUFF DEV FDN

Scoltsbluff, NE

SCOTTSBLUFF GEHRING PAYROLL

Development Foundation

Scoltsbhuff, NE

290380-0001-4

SCREENSCOPE INC

Arlington, VA
$277360-0001-8$

$278390-9001-0$

$278400-6101-7$

$2784100(0) 1-4$ 
Awardee $\quad \begin{gathered}\text { Identification } \\ \text { code }\end{gathered}$

SCULLY CAPITAL

Washington, DC

$291070-0001-4$

SE INC

Deaver, WY

190870-0011-]

Lingle, WY

$190870-0012-\mathrm{X}$

SE MANUFACTURED HOUSING ALL

New York, NY

$279590-0001-8$

SE MICHIGAN COUNCH OF GOVT

Detroit-Toronto Clm Cities Cor

Deroit, MI

286170-0101-1

SE MO REG PLAN \& ECON DEV COMM

Penyvílle, MO

279190-0001-7

SEA SOLAR POWER INC

York, PA

278420-0001-8

SEAGER JON W

Plalinum, AK

278430-0001-1

SEATTLE COALITION FOR EDUC EQU Stattle, WA

$288970-0001-0$

SEATTLE METROCENTER YMCA Sealte, WA

$278440-0001-5$

SEAWAY PIPELINE CO

Barlesville, OK

Freeport, TX

$0 / 4050-0001-0$

$074050-(0002-9$

SECME DNC

Atlanta, GA

SECURE COMPUTING CORP

Roseville. MN

SECURE SERVICES TECHNOLOGY

Chantilly, VA

234690-0001-8

$290260-0001-4$

SECURUS SYSTEMS

Denver, $\mathrm{CO}$

Montrose, $\mathrm{CO}$

SETTZ NEIL

Villa Grove, CO

SELF RELIANCE FOUNDATION

Trampas, NM

SENCENBAUGH WIND ELEC

Mountain Vjew, CA

SENIOR CITIZENS ASSOC

Missoula, MT

SENSOR TECHNOLOGY INC

Chatsworth, CA

SENTOR INC

Mechanicsville, VA

SENTROL INC

Tualatin, $O R$

SEREF

Washington, DC

SERVICE-HANSHEW

Eaergy Resource Center

Anchorage, AK

SETAC

Stillwater, OK

$156400-0002-3$

$292130-0002-2$

292130-0001-4

$275110-0001-0$

$074160-0003-3$

$278450-0001-9$

$278460-0001 \cdot 2$

$278470-0001-6$

288640-0001-1

$284060-0001-5$

$284250-0001-7$

$278480.0101-6$

$278490-0001 \cdot 3$

SETON JOHNSON \& ODELL INC

Fortland, OR

$163240-0002-8$

SETS INC

Honoluiu, HI

$278500-0001-3$
Awardee

Identification

code

SEWARD HIGH SCHOOL LIBRARY

Seward, AK

$278510-0001-7$

SEYMOUR COMMUNITY SCHOOLS

Seymour, WI

$278520-0001-0$

SEYMOUR WERNER

Silver Spring, MD

281990-0001-5

SHAFFER JANE

Heath, OH

$278530-0001-4$

SHAH \& ASSOC INC

Gaithersburg. MD

J09940-000I-4

SHANA CORP

Golden, $\mathrm{CO}$

$283090-0001-4$

SHANKS EXCAVATING

Durant, OK

287240-000]-9

SHAPIRO ASSOC

Stattle, WA

SHARP ELECTRONICS CORP

Golđen, $\mathrm{CO}$

Mahwah, NJ

SHASTA COUNTY COMM ACT Redding, CA

SHAWNEE SOLAR PROJECT

Carbondate, IL

SHEEHAN HIGH SCHOOL

Wallingford, CT

SHEET METAL \& AC CTRS NA Vienna, VA

$278540-0001.8$

074540-0006-1

(1)74540-0002-9

278550-0001-1

$278560-0001-5$

$278570-0001-9$

$27858000001-2$

SHELDAHL INC

Northfield, MN

SHELDON LEON BIERMAN

Wașhington Grove, MD

278590-0001-6

$278600-0001-6$

SHELL PIPE LINE CORP

Baton Rouge, LA

$275900-0003.5$

$2759000001-9$

New lberia, LA

$275900 \times 0002-7$

SHELTON WOOD ENERGY RESEARCH Santa Fe, NM

SHEPARD MD GINANDES

Kingshill, VI

$278610-000 \mathrm{~J}-\mathrm{X}$

SHEPHERD DENNIS

Maple Hill, KS

$278650-0001-4$

SHERMAN GERBER ASSOC

$278620-0001-3$

Northbrook, IL

$265690-0001 \cdot 2$

SHIELDS CONSTR CO

Atlanta, GA

$278640-0001-0$

SHIH S C H

Raleigh, NC

SHIRLEY JEFF

Columbia, sc

SHOSHONE-BANNOCK SCH DIST $\$ 512$

Fort Hall, ID

SICAME CORP

Cinçinnati, $\mathrm{OH}$

SIEHE GOVERNMENT SERVICES

Grand PTajie, TX

SIEMENS

Las Vegas, NV

$277980-0001-1$

270600-0002-1

$279740-0001-5$

$292320-0001-6$

$291820-0001-8$

$190410-0003-8$ 


$$
\begin{array}{cc}
\text { Identification } \\
\text { code }
\end{array}
$$

SIEMENS \& ALLIS INC

Richland, MS

SIEMENS POWRR T $\&$ D CO INC Charlalte, NC

Jackson, MS

$075110-0004-5$

$285970-0001-5$

285970-0002-3

SIEMENS WESTINGHOUSE PWR CORP Pittsbirgh, PA

$29033040001-6$

SIENNA TECHNOLOGIES INC

Woodinyille, WA

SIGNATRON TECHNOLOGY CORP

Concord, MA

$284140-0001-0$

SILBERNAGLE LARRY

South Sioux City, NE

SILER WLLIAM

Bonners FerTy, [D

SILICON POWER CORP

Malvent, PA

SILICON TECH CORP

Oakland, NJ

SILTEC CORP Menlo Park, CA

SILVER STATE DISPOSAL

Las Vegas, NV

SMMETRLX INC Troy, NY

STMMONS ELECTRIC

Englewood

Golden, $\mathrm{CO}$

SIMONS DR WILLIAM

Morgantown, WV

SIMPSON FRANK A

Anchorage, AK

$284340-0001-6$

$271990-0001-4$

$282360 \cdot 0006]-X$

$285350-0001-1$

278670-(0001-1

278680-0001-5

$29] 410-0001-3$

$285720-000]-1$

SIMPSON STRATTA AND ASSOC San Francisco, CA

SIMSBURY BOARD OF EDUCATION Simsbury, CT

SIMULATION PHYSICS INC

Bedford, MA

Burlington, MA

SIMULATION TECH \& APP RES INC Mequon, WI

SINAI HOSPITAL OF DETROIT

Detroit, MI

SINGER CORP

Fairfeld, NJ

Silver Spring, MD

R \& D Lab

Fairfield, NJ

SINGMASTER \& BREYER INC New York, NY

SINZ E

Miami, FL

SIPE JOHN PAUL

Manchester. TN

SISTERS OF ST DOMINIC

Dimensions at Marymount

Twoma, WA

$075400-0003.1$

$075400-0002-3$

075420-(0001-2

$265610-0001-3$

270880-0001-6

$220230-0102-1$
Awarde

Identiflcation code

SIZEMORE \& ASSOC

Atlanta, GA

$278740-0001-3$

SKAGIT COLNTY OF

Planning Commiksion

Mt Vernon, WA

278750-0101-3

SKF INDUSTRIES INC

Lubbock, TX

$075570-0002-1$

SKINNER FERRELL $S$

Iuka, MS

SKINNER GEORGE

Washington, DC

SKIPPER RALPH s

Shugualak, MS

SKK INC

Rosemont, IL

SKTEL CORP

Golden, CO

SKYTEL FEDERAL SALES

Washington, DC

SKYTEL GOVERNMENT SALES

Folsom, $\mathrm{CA}_{\mathrm{A}}$

Wayne, N!

SLACK ASSOCS

Baltimore, MD

SLOAN R T

Hamillon, MO

SMALL BUSINESS ADMINISTRATION

Denver, $C O$

Pittshurgh, PA

Washington, DC

AMTAR

Atlanta, GA

Geo-Life

Puyallup, WA

Max G Rivera Constr Co

Los Alamos, NM

Montegut \& Rabb CPA

New Orleans, LA

Washington District Office

Washington, DC

SMALL CHANGE ORIG THIR

Minneapolis, MN

$267050-0009-5$

$2680020-0001-6$

$278760-0001-0$

131270-0001-4

$287150-0001-\mathrm{X}$

292570-0001-X

286630-0001-4

$286630-0002-2$

$278770-0001-4$

277150-0001-9

075670-0009-1

75670-0010-5

$075670-0003-2$

$075670-0312-0$

075670-0108-X

$075670-0413-5$

$075670-0211-6$

$075670-0503-4$

$278780-0004-8$

SMALL ROBERT

Del Mar, CA

SMALL TRIBES OF WESTERN WA

Sumner, WA

277550-0001-X

075400-0103-8
SMART START CHILD CARE CENTER

Los Vegas, NV

SMARTNET WORKS

Leesburg, VA

SMARTPLUG INC

Sarasola, FL

SMARTSTAR CORP

Oolela, CA

SMEDLEY ROBERT $W$

Lituleton, $\mathrm{CO}$

SMEED COMMUNICATION SERVICES

Eugene, OR 
Identification
cude

SMITH LOREN

Great Falls, MT

SMITH MILLER \& ASSOC

Kingston, PA

\$MITH ROD FREEBAIRN

Muir Beach, CA

SMPTH THOMAS E

Lithus, $\mathrm{HI}$

SMITHSONIAN INSTITUTION

Sponsored Programs Office of

Washington, DC

SMITHSONIAN SCIENCE INFO

Washington, DC

$272510-0001-6$

$264980-0001-5$

$277630-0001-5$

278800-000L-1

$075820-1303.4$

$278810-0001-5$

SMITHTOWN CENTRAL \$CHOOL DIST

Smithtown, NY

SNITZLER JAMES R

Camp Springs, MD

075840-0002-9

SOBOTKA \& CO INC

Washington, DC

$278820-0001-9$

SOBRAN INC

Daylon, $\mathrm{OH}$

SOCIAL RESEARCH APPLIC CORP

Washington, DC

SOCIETE GENERALE TECH NOUVELLE

Si Quentin Yvellines Cedex, France 267170-0001-5

SOCLETY FOR OCCUR \& ENY HEALTH

McLean, VA

$275880-0001-5$

SOEHNGEN \& ASSOCS

Fairborn, OH

$278840-0001-6$

SOFREGAZ US INC

Houston, TX

SOFTMART INC

Downingtown, PA

$075970-0002-2$

246850-0002-1

075990-0002-X

SOFTSMITH INC

Houston, TX

$285650-0000]-X$

$158960-0003-0$

$291710-0001-1$

SOFTWARE AG OF NORTH AMERICA

Resion, VA

SOFTWARE SPECTRUM TECH SV GROU

Greenwood Village, CO

SOIL-CRETE SOLAR CO

Navajo Dam, NM

SOL-AIRSEARCH INC

Branford, CT

SOL/LOS INC

Los Angeles, CA

SOLAMAT INC

Bartington, RI

SOLAR ACTION INC

Washington, DC

SOLAR ARCH \& SYS DESIGN

Auburr, AL

SOLAR CONTROL CORP

Boulder, $C O$

SOLAR ELECTRONICS INC

Bellingham, MA

SOLAR ENERGY APPLICATION

Douglas, AZ
287260-000J-6

$278860-0001 \cdot 3$

278870-0001-7

$278880-9001-0$

$076160-0002-9$

$2788900001-4$

$278900-0001-4$

278910-0001-8

278980-0001-3

$264620-0001-5$
Awardee

Identification

code

SOLAR ENERGY DIV OF

Washington, DC

SOLAR ENERGY PRODUCTS CO

Avor Lake, $\mathrm{OH}$

SOLAR ENGR CO

Casselberty, FL

SOLAR ENVIRONMENTAL ENG CO

Fort Collins, $C O$

SOLAR GREENHOUSE ASSOC

Wchster Groves, MO

SOLAR HEATING \& COOLING INC

Bi]oxi, MS

SOLAR RATING a CERTIFIC CORP

Cocos. FL

Washington, DC

SOLAR KESOURCES INC

Los Angeles, CA

Taos, NM

SOLAR ROOM INC

Taos, NM

SOLAK STORE

Peoria, IL

$264650-0001-6$

264630-0101-5

264640-0001-2

076310-0001-8

278920-00001-1

278660-0001-8

$076370-0002-8$

$076370-0001-X$

$076400-0001-7$

$076400-0002-5$

$278930-0001-5$

$278940-0001-9$

SOLAR SUTTES

Indianapoliss, IN

SOLAR SYSTEMS DESIGN INC

Selkirk, NY

SOLARAMICS INC

El Segundo, CA

SOLAREX CORP

Frederick, MD

Rockford, MD

Rockville, MD

SOLARGENICS INC

Chatsworth, CA

SOLARTEC INC

Abilene, TX

SOLATION PRODUCTS INC

York, PA

SOLDOTNA PUBLIC LIBRARY

Soldotna, AK

SOLERGY

San Francisco, CA

SOLVA-TEK ASSOCS

Topsfield, MA

SOLVEWARE SYSTEMS INC

Columbus, $\mathrm{OH}$

SOMERSET COUNTY PARK COM

Somerville, NJ

SONALYSTS INC

Waterford, $C T$

SONESON SIGFRIED \& JUDITH

Bryn Athyn, PA

SONOMA COUNTY OF

Sonoma, CA

$278950-0001-2$

$278960-0001-6$

$278970-0001+x$

$076470-0003.9$

$076470-0002-0$

(7)6470-0001-2

278990-0001-7

$279000-0001-1$

$279010-0001-5$

$279020-0001-9$

$279030-0001-2$

$279040-0001-6$

$279050 \cdot 0001-\mathrm{X}$

$279060-0001-3$

279070-0001-7

279080-0001-0

SONOMA STATE UNIV ACAD FDN INC

Rohnert Park, CA

SONOTONE CORP

Los Angeles, CA
279090-0001-4

279100.0001-4

$279110-0001-8$ 
Awardee

Identification

code

SONSIGHT PROJECTS INC

Adelphi, MD

SOUNDVISION PRODUCTIONS

Berkeley, CA

SOURCE ASSOC.

Silver Spring. MD

SOURCE ONE SUPPLY LTD

Broomall, PA.

SOUSA STEPHEN

Hickory, NC

SOUTH CAROLINA STATE OF

Columbia, SC

Commerce Dept of

Columbia, SC

DEV BD / Dir of Geology

Columbia, SC

Emergency Preparedness

Columbia, SC

Governor Ofice of Eeron Div

Columbia, SC

Governor Office of the

Columbia, SC

Law Enforcement Đivision

Coluribia, SC

Natural Resources Dept of

Columbia, SC

Recreation \& Tourism Dept of

Columbja, SC

State Energy Office

Columbia, SC

Washington D C Office

Washinglon, DC

SOUTH CAROLINA UNIVERSTTIES

Research \& Education Fnd

Clemson, SC.

SOUTH CAROLINA UNIVERSITY OF

Archeology \& Anthropology

Columbia, SC

SOUTH COAST TECHNOLOGY INC

Santa Bartara, CA

SOUTH COLUMBLA BASIN IRRI DIST

Pasco. WA

SOU'H DAKOTA COOP ORGANIC FUEL

Committee

Brookings, SD

$279180-0101-\mathrm{X}$

SOUTH DAKOTA SCHOOL OF MINES

Pierre, SD

076850-0003-2

Mines \& Technology

Rapid City, SD

$076850-0401-1$

SOUTH DAKOTA STATE OF

Socisl Service Dept of

Pierre, SD

$076860-0701-4$

Weatherization

Pierre, SD

$0768601001-5$

SOUTH FLORIDA UNIVERSITY OF

Marine Sciences

Si Petersburg. FL

$076920-0503-6$
Awardee

Identification

code

SOUTH LEMHI SCHOOL DIST \$292

Leadore, ID 283620-0001-5

SOUTH PUGET SOLND SOLAR ENERGY

Olympia. WA.

279160-000)]-6

SOUTHEAST ELEC

Rel Council

Birminghan, AL

279170-0101-6

SOUTHEASTERN ENV RESOURCES ALL

Aiken, SC

$241100-(0002-5$

SOUTHEASTERN EXPEDTTIONS

Clayton, GA

279200-0001-7

SOUTHERN CA SOLAR ENERGY ASSOC

San Diego, CA

SOUTHERN CALIFORNIA EDISON CO

Customer Solutions Business

San Dimas, CA

$077230-0303-x$

Editon International Co

Rosemead, CA

SOUTHERN CALIFORNIA GAS CO

Los Angeles, CA

$077230-0201.7$

SOUTHERN CALIFORNIA UNIV OF

Los Angeles, CA

SOUTHERN CTR FOR ELECT ENGR

Ortando, FL

$131310-0002-3$

GOUTHERN ECONOMIC RES ASSOC

Hilton Head lsland, SC

SOUTHERN ILLNOIS UNIVERSITY

Board of Trustees

Carbondale, IL

$077310-2401-6$

Coal Technolory Lab

Carbondale, IL

$0773(0-250)-2$

SOUTHERN INTEREST NUCLR BD

Atlanta, GA

$279230-0001-8$

SOUTHERN MISSISSIPPI UNIV OF

Chemistry Dept

Hatiesburg, M5

$077340-0101-7$

Physical Plant Diviston

Hattiesburg, MS

077340-0401-6

SOUTHERN OHIO DIVERSIFICAN INI

Piketon, $\mathrm{OH}$

$287630-000]-6$

SOUTHERN OREGON STATE COLLEGE

Eugene, $O R$

279240-0(9)1-1

SOUTHERN SCIENCE APPLICATIONS

Dunedin, FL

$077400-0001-9$

SOUTHERN SOLAR ENERGY CENTER Atlanta, GA

$279250000 \mathrm{~L}-5$

SOUTHERN STATES ENERGY BOARD

Research \& Development

Atlantin, GA

077410-0101-9

SOUTHERN UTE INDLAN TREBE

Energy Resource Oflice

Ignacio, $\mathrm{CO}$

SOUTHLAND ROYALTY CO

$077450-0101-3$

Fort Worth, TX

$279260-0001-9$

SOUTHWEST COMMUNITY OUTREACH

Denver,

$283870-0001-9$

SOUTHWEST FOUND FOR BIOMED RES

San Antonio, TX

$284760-0001-4$ 
Awardee $\quad \begin{gathered}\text { Identifieation } \\ \text { code }\end{gathered}$

SOZA \& CO LTD

A.rlington, $V A$

SPACE SCIENCE INSTTTUTE

Silver Spring, MD

SPACE WORK INC

Colorado Springs, $\mathrm{CO}$

$247440-0003-7$

$278270-(0001-0$

$264810-0001-7$

SPANCRETE INDUSTRIES INC

Milwaukee. WI

SPARHAWKE ENTERPRISES INC

Richland, WA

$279270-0001-2$

$287340-0601-1$

SPB MGMT CONSULTING GROUP INC

Richland, WA

$285200-0001-0$

SPEARS \& ASSOC INC

Tulsa, OK

077930-000L-3

SPECTRA CORP

Lawence, MA

$284650-0001-8$

SPECTRA RESEARCH SYSTEMS

Newport Beach, CA

SPECTRO-SYSTEMS INC

Spritgfield, VA

SPECTROLAB INC

Sylmar, CA

SPECTRON DEVELOPMENT LABS INC

Costa Mesa, CA

SPECTRUMEDIX CORP

State College, PA

SPELMAN COLLEGE

Atlanta, GA

SPERRY RESEARCH CENTER

Sudbury, MA

SPIEGEE WILLIAM $M$

Arcadia, FL

SPIN LOGIC INC

San Diego, CA

SPOKANE COUNTY OF

Building \& Safety Dept

Spokane, WA

SPOKANE R-VII SCHOOL DISTRICT

Spokane, MO

SPRIE CORP

Bedford, MA

$279290-0001 \cdot \mathrm{X}$

279300-0001-X

018020-0001-7

284330-0001-2

$247220-0001-8$

$279310-0001-3$

$289540-0001-0$

$283250-0001-5$

[ I 7340-0201-1

282910000]-8

$279320-0001.7$

SPRINGBORN LABORATORIES INC

Enfield, CT

$078220-0001.2$

SPRINGBORN TESTING \& RESEARCH

Enfield, CT

\$PRINGFIELD WORKSHOP INC

Springfield, MO

$285090-0001-8$

SPRINT COMMLINICATIONS

Hemdon, VA

$245060-000 \mathrm{~L}-3$

SQUARE D CO

Denver, $\mathrm{CO}$

SRC INTERNATIONAL INC

Cost Co

Denver, $\mathrm{CO}$

SRI INTERNATIONAL

Menlo Park, CA

Chentical Industries Div

Menlo Park, $\mathrm{CA}_{\mathrm{A}}$

251610-0001-5

$078280-0003-0$

279330-0101-7

078330-0001-9

$978330-1401-X$
Awardee

Identification

corde

Eneray Center

Menlo Park, CA

Ltfe Sciences Div

Menio Park, CA

078330-1501-6

$078330-0601-7$

SRT GROUP INC

Miami, FL

$290000-0001-7$

SSDS INC

Fairfax, VA

ST ANTHONY'S HOSPITAL INC

St Petersburg, FL

ST BONAVENTURE UNIV

Physics

St Bonaventure, NY

ST CHARLES SCHOOL

Arlington, VA

ST CLEMENT PARISH

San Carlos, CA

ST CLOUD UNITARTON

St Cloud, MN

ST CROIX CENTRAL HIGH SCHOOL

Charlotte Amalie, V]

ST DAVID UNIFIED SCH DIST \$21

Si David, AZ

ST JOE SCHOOL DISTRICT

St Joc, AR

ST JOHNS' UNION HSD \#1

St Johns, AZ

275050-0001-2

$267140-0001-4$

078770-0101-0

$184720-0002-8$

$163290-0002-6$

279340-0001-4

$279350-0001-8$

$267180-0001-9$

$267190-0001 \cdot 2$

ST JOSEPH COLJEGE

Easi Hartford, CT

West Hartfond, CT

ST JOSEPH HEALTH CENTER

St Charles, MO

ST JOSEPH'S CSSR SCHOOL

Arch-tiocese of Denver

Denver. CO

ST JOSEPH'S PARISH

Bishop of Salina Diocese

Hays, KS

ST LOUIS UNIVERSITY

St Louis, MO

$173580-0001-3$

$079870-0003-5$

079870-0004-3

$282920-0001-1$

ST LUKE'S HOSPITAL

Boise, ID

098480-(1)101-8

080090-0101-5

$080240-0001-6$

ST MARGARET OF CORTONA SCHOOL

New York, $N$ Y

$279360-0001-1$

ST MARIES JOINT SCH DIST \$41

St Maries, ID

$283500-0001.5$

ST MARY SCHOOL

Norwalk, $\mathrm{OH}$

$080460-0009-4$

ST MARY'S AREA SCHOOL DISTRICT St Marys, PA $279380-0001-9$

ST MARY'S COLLEGE OF MARYLAND St Marys City, MD

279390-0001-2

ST MARY'S HIGH SCHOOL

St Marys, AK

0805 70-0002-3

ST MAKY'S HOSPITAL

East Saint Lotuis, Il

$080590-0019-5$

ST MARY'S SCHOOL

Baltimore, MD

080700-0024-4 
Awardee

Identification code

ST MARY'S \$CHOOE DISTRICT St Marys, AK

ST NICHOLAS SPEECH \& HEAR Greenwood, SC

ST PATRFCK CATHOLJC SCHOOL

Fond Du Lac, WI

ST PAUL CITY OF St Paul, AK

ST PAUL LUTHERAN SCHOOL Shellors, NE

ST PAUL PUBLIC SCHOOLS St Paul, AR

ST PETERSBURG CITY OF Largo, FL St Petersburg, FL

ST TERESA'S SCHOOL Lincoln, NE

ST VINCENT'S MEDICAL CENTER New York, NY

ST VINCENTS Consell, WA

STA-COOL HEATING \& AC INC Clinton, MD

STAMET INC Gardena, CA

STANA JAMES $M$ Longwood, FL

STANDARD \& POOR'S DRI Washington, DC

STANDARD OIL CO Chicago, il

STANFORD UNIVERSITY Palo Alto, CA Stanford, CA

Chemistry Dept of Stanford, CA

Materials Science \& Eng Dept Stanford, CA

Materials Science Dept Stanford, CA

NIV Dept of Materials

Stanfort, CA

NVR

Stanford, CA

Petroleum Engineering

Stanford, CA

Plasma Research Institute for Stanford, CA

Res Proj Office Stantord, CA

Sponsors Proj Office Stanford, CA

Systems Optimization Lab Stantord. CA

Trustees Board of Stanford, CA

STANLEY CONSULTANTS INC Washington, $D C$
$279400 \cdot 0001.2$

$279470-000]-8$

$165520-0002-2$

$267750-0001-4$

$081010-0004-6$

$241820-0001-3$

$267150-00002-6$

267150-0001-8

$282820-0001-9$

$081770-0002-0$

279480-0001-1

$229190-0001-2$

215340-0002-X

$270490-0001-9$

$293130-0001-6$

$118030-0001-5$

$081920-0002-8$

$081920-000] \cdot \mathrm{X}$

$081920-050]-1$

$081920-3601-4$

$081920-3701.0$

$061920-3401-1$

$08[920-350]-8$

$081920)-30(01-6$

$081920-1401-0$

$081920-380]-7$

$081920-3301-5$

$081920-3901-3$

$081920-4001-1$

081940-0003-3
Awardee

Identification code

STANTON KAY \& WATSON

San Francisco, CA

STARKEY VONLEY

Kissimmee, FL

STARMET CORP

Bamwell, SC

STARR GLENN

Bellingham, WA

STARY MARVIN

Clasemont, CA

$265320-0001-9$

STAT-A-MATRIX

Edison, NJ

STATE CORRECTIONAL CENTER Eagle River, AK.

STATE DEPARTMENT OF

US Embassy

Mexico City, Mexico

$082060-0608-7$

STATENS INDUSTRIVERK Stockholm, Sweden

STATEWIDE AUTOMATIC FIRE ELEC Las Vegas, NV

STATISTICA INC

Gaithersburg, MD

$2816000001-4$

$292200-0001-6$

$267640-000]-8$

$279790-0001-3$

$291060-0001-0$

279800-0001-3

STAUFFER CHEMICAL CO

Indostrial Chemicols

Westport, CT

STEARNS CATALYTIC CORP

Power Division

Denver: CO

279660-0001-X

291090-0001-1

$291850-0001-9$

STEARNS-ROGER SERVICES INC Denver, $\mathrm{CO}$

35980-0]01-0

STEEL MANUFACTURES ASSOCIATION Washington, DC

STEEN GARRY W Cheyenne, WY

STEIN RICHARD G New York, NY

STEINECKER CYNTHHA H Beaver, WA

STELLE GROUP Stelle, IL

279810-0101-3

STEPHENS JOHN

Davis, CA

STEPHENSON ROY L Bogarl, GA

STERLING COMMERCE INC Reston, VA

STERLING HOBE CORP Washington, DC

STERLENG INSTITUTE Washington, DC

STERLING PARK DISTRICT Sterling, IL

STEVENS INSTITUTE OF TECH Hoboken, NJ

STEVENS POINT AREA COOP Stevens Poinl, WI
289690-0001-1

267790-0001-9

$279830-0001-4$

$279840-0001-8$

279850-0001-1

2709]0-0001-3

$279870-0001-9$

$287760-0001-\mathrm{X}$

$279880-0001-2$

$279890-0001-6$

$279900-0001-6$

$082260-0001-7$

118100-0001-7 
Awardee

Identification

code

STEWARD MARY A \& BETHEL

Lawrence, KS

STEWARD W GENE

Boulder, $\mathrm{CO}$

STEWART DONALD R

Wichita, KS

STEWART ED

Englewood, $C O$

STIM-LAB INC

Duncan, OK

STIRLING TECHNOLOGY CO

Kennewick, WA

NOEN

Kennewick, WA

STN INTERNATIONAL

Columbus, $\mathrm{OH}$

STONE \& WEBSTER ENG CORP

Evans, GA

STONE FAMILY DULCIMERS

Montrose, PA

STORM RESEARCH INSTITUTE FOR

Houston, $\mathrm{TX}$

STRAHLE JIM B

Iola, KS

STRAIN MONITOR SYSTEMS INC

San Diego, CA

STRATEGIC \& INTL STUD CTR FOR

Washington, DC

284770-0001-8

STRATEGIC ENVIRONMENTAL ASSOC

Underwood, WA

$289910-0001-0$

STRAUSS PHOTO-TECHNICAL SVC

Washington, DC

STRECHER ELECTRICAL EQUIP CO

Beyne Heusay, Belgium

082530-10001-4

STROMSETH CONSTRUCTION

Groton, SD

279970-0001-1

Watertown, SD

$291950-0001-1$

291950-0002-X

STRUBBLE V

Raleigh, NC

281400-0001-9

STRUCTURAL MECHANICS ASSOC

Newport Beach, CA

STRUCTURAL RESEARCH INC

Mardison, WI

STRUCTURES UNLIMITED INC

Manchester, NH

STUART C IRBY CO

Idalia, MO

279980-0001-5

$279990-0001-9$

$280000-0001-6$

STUART HIRSH/HIRSH PROFESSNL

Anchorage, AK

STV ENGINEERS INC

Pottsiown, PA

SUETERRANEAN RESEARCH INC

Burlington, VT

SUD ASSOC

Atlantic Beach, NC

Chicago, IL

Cleveland, $\mathrm{OH}$

Detroit, MJ
$264000-0002-\mathrm{X}$

287790-0001-0

$082720-0001-6$

285440-0001-0

$202970-00160$

202970-0014-4

202970-6013-6

202970-0015-2
Awardee

Identification code

Duluth, MN

202970-0012-8

Milwaukee, WI

$202970-0010-1$

Ramey, PR

$202970-0009-8$

South Bend, IN

$202970-0011 . \mathrm{X}$

SUGAR-SALEM JOINT DIST \# 322

Sugar Ciry, ID

SULLIVAN J W

Derby, KS

SUMME \& FRANKENSTEIN

Hamilton, $\mathrm{OH}$

SUMMERS COUNTY SERVICE COUNCIL

Hinton, WV

$264870-0001-9$

$270310-000 !-7$

SUN GARD COMPUTER SERVICE

Herndon, VA

$280020-0001-3$

SUN MICROSYSTEMS FENERAL INC

McLean, VA

290880-0001-8

SUN MICROSYSTEMS INC

Chelmsford, MA

234960-0002-3

SUN SYSTEMS INC

Boston, MA

SUN TECH ENERGY CORP

Pauls Valley, $O K$

181980-0003-8

UN/REP

Atlanta, GA

SUNCOAST PIMEG INC

St Petersburg, FL

SUNDSTRAND ENERGY SYSTEM

Rockfond, IL

$280030 \cdot 0001-7$

$280040-0001-0$

280050-0001-4

$249500-0001-2$

280060-0001-8

SUNFLOWER COUNTY LIBRARY

Indianola, MS

280070-0001-1

SUNFLOWER SOLAR INC Collcge Park, GA

$280080-000 \mathrm{l}-5$

SINPOWER INC

Athens, OH

SUNRISE ENERGY PRODUCTS

Pellsion, Ml?

SUNSHINE CHEMICAL SPECIALTIES

Cherry Hill, NJ

SUNSHINE CLEANING SERVICES

Della, CO

$288470-0001-7$

SUNSHINE INSULATION

A.buquerque, NM

$280110-0001-2$

SUNTECH INC

Richardson, TX

SUNTEK RESEARCH ASSOC

083220-0003-0

Corke Madera, CA

$083230-0001-8$

SUNWIND LTD

Sebastopol, CA

SUPER COMP AUTOMOTIVE APPL

Southfield, MI

$280120-0001-6$

SUPERINTENDENT OF DOCUMENTS

London, United Kingdom

SUPERIOR TILE COMPANY

Oakland, CA

SUPERMARKET COMMUNICATIONS

Stanford, CT

SUPERWAVE TECHNOLOGY INC

Santa Clara, CA

280160-0001-0 
Awardee $\begin{gathered}\text { Identification } \\ \text { code }\end{gathered}$

SURTON CORP

Fairfax, VA

$280170 \cdot 0001-4$

SUSTAINABLE ENERGY ASSOCIATTON Greenfield, MA

SV SYSTEMS LLC

Warwick, NY

$292910-0001-9$

SVERDRUP \& PARCEL ASSOC INC

St Louis, MO

SVT ASSOC INC

Eden Prairie, MN

280190-0001-]

SWEDISH MEDIC

$285120-000]-5$

Seatlle, WA

SWEDLOW INC

Garden Grove, $\mathrm{CA}$

SWEETWATER CTY SCHOOL DIST \#2

Green River, WY

SYBASE INC

Concord, MA

SYLCO INC

Kenneth A Sylvester

Berlin, MD

SYLVEST MGMT SYSTEMS CORP

Greenbelt, MD

Landover, MD

227 140-0003-7

SYMBIONT

Washington, DC

SYMYX TECHNOLOGIES

Sunnyvale, $\mathrm{CA}$

SYRACUSE UNIVERSITY RES CORP Syracuse, NY

SYSTEM ENERGY RESOURCES INC

Mississippi Power \& Light Co

New Orleans, LA

SYSTEM SCIENCES INC

Bethesda, MD

SYSTEMETRICS INC

Blacksburg, VA

280230-0101-9

.

$228750-0005-5$

$228750-00047$

$286100-0001-\mathrm{X}$ SYSTEMS \& PROCESSES ENG CORP
Auștin, TX

SYSTEMS CONSULTANTS INC Washington, $D C$

SYSTEMS CONTROL INC

Birmingham, AL

Palo Alto, CA

Environmental Engineering

Anaheirn, CA

SYSTEMS INDUSTRIES INC

Albuquerque, NM

Golden, $\mathrm{CO}$

Kirkland, WA

Lakewood, CO

Milpitas, CA

Pittshurgh, PA

Portland, $O R$

SYSTEMS MANAGEMENT ASSOC

Washington, $D C$

SYSTEMS SCIENCE \& SOFTWARE

La Jolla, CA

$283820-0001-0$

280240-6001-6

083710-0001-4

$083720-0003-4$

$083720-0002-6$

083720-0101-4

$083750-0007-8$

083750-0004-3

$083750-0006-\mathrm{X}$

$083750-0001-9$

083750-0005-1

$083750.0009-4$

083750-0008-6

083760-0002-0

$083770-0002-4$
290050-0001-5

Identification
code

Awandee

SYTEL ENC

Bethesda, MD

278320-0001-5

$\mathbf{T}$

$T$ \& R SERVICE CO

Sioux Falls, SD

259160-0002-2

T \& R TRUCKING INC

Glasgow, MT

T F POWERS CONSTRUCTION CO

Fargo, ND

T-COM CORP

Mountain View, CA

TAHOMA FOOD SYSTEM

Tacoma, WA

TALCOTT MNT SCI CTR FOR STUD

Avon, CT

TALLEY FRAC CORP

Mesa, AZ

$291960-0001-5$

$290140-0001-4$

TASC

Restion, VA

TCB INDUSTRIAL INC

Modesto, CA

$264720-0001 \cdot X$

TEACO INC

Spartanburg, SC

TECHNOLOGY ADVISORS INVESTMENT

Golden, CO

290770-0001-1

$284220-(000)-6$

2805 10-0001-3

$084130-0002-5$

286270-0001-8

$2862000001-1$

TECHNOLOGY CONSULTANTS INC

Knoxville, TN

$283900-0001-6$

TECHNOLOGY

$285500-0001-9$

Kingwood, TX

TECHNOLOGY MATREX CORP

Syracusse, NY

TECHNOLOGY PLANNING \& MGMT

Firfax, VA

TECHRITE

Marblehead, MA

TEI ANALYTICAL INC

Washington, PA

TELEDYNE BROWN ENGINEERING

Hunt Valley, MD
TELEDYNE POWER ENGINEERING

Hunt Valley, MD

TELLINGHUISEN INC

Brandon, SD

Huron, SD

Summit, SD

TEMPRESS TECHNOLOGIES INC

Kent, WA

TENNESSEE STATE OF

Human Services Dept of

Nashville, TN

TENNESSEE TECHNOLOGICAL UNIV

Cookeville, TN

TENNESSEE UNIVERSITY OF

Knoxville, TN

Oak Ridge. TN

Eaergy Environment Res Ctr

Knoxville, TN
105040-0002-0

$287920-0001-0$

292600-0001-7

$287510-0001-6$

$280410-0001-0$

084530-0002-6

$275060-0001-6$

$257460-0004-3$

257460-0003-5

$257460-0005-1$

285510-0001-2

$084790-1004-5$

$0848100001-9$

$084820-0002-0$

$084820-00 \times 7-1$

$084820-3002-7$ 

Awardee
Identification
code

Research Office of

Knoxvilie, TN

$084820-3] 02-3$

TENNESSEE VALLEY AUTHORITY

Atmospheric Sciences

Muscle Shoals, AL

084830-1203-0

TERA ECONOMIC RES ASSOC

Arlington, VA

$280530-0001-0$

TERA INC

Falts Church, VA

084870-0002-9

TERMINAL EXCHANGE SYSTEM

Denver, $C O$

284050-0001-1

TERMINATE NETWORK LEASING CORP

Silver Spring, MD

$280540-0001-4$

TERRA TEK INC

Jackson, TN

Salt Lake City, UT

Unaversity Research Park

Salt Lake City, UT

TERRADEX CORP

Glenwood, II

TERRADIGM INC

Albuquergue, NM

TERRASUN LLC

Tucson, AZ

TERRY CLAUDE

Allanta, GA

TERRYS INTERSTATE

Tyle, TX

TESH

Coeur $D^{+}$alene, iD

TESKE DR C E

Teras Tech University

Lubbock, TX

TETRA TECH INC

Ouk Ridge, TN

TEXACO PANAMA INC

Panana 3, Pitcairn

TEXACO REFINING \& MARKTG INC

Costa Mesa, CA

Houston, TX

Long Beach, CA

Tulsa, $\mathrm{OK}$

Universal Cily, CA

TEXACO TRADING \& TRANSP INC

Burbank, CA.

084920-0001-5

084920-0201-8

$181510-0002-3$

$291040-0001-3$

$285100-0001-8$

$280550-0001-8$

$280560-0901-1$

$280580-0001.9$

$265150-0 \mathrm{~J} 0 \mathrm{~L}-0$

$085000-0007-4$

$085050-0003-\mathrm{X}$

$1650-0007-8$

161950-0006-X

161950-0005-1

161950-0004-3

$161950-0002-7$

TEXAS A \& M RESEARCH EON

College Station. TX

1570]0-00004-4

TEXAS A \& M UNIVERSITY

College Station, TX

Chemitstry Dept

College Station, TX

Petroletm Engineering

College Station, TX

TEXAS EMERGY ADVISORY COUNCIL

Austin, TX

TEXAS ENG EXPERIMENT STATION

Golden, $\mathrm{CO}$

$033220-0001-\mathrm{x}$

$085070-0001-0$

085070-0301-X

$085070-1301-5$

$280590-0001-2$

$187400-0002-8$
084920-0003-1

Awardee

Identification

code

Energy Syst Lab/Tues/Tamus

College Station, TX

Res Svcs /Sponsored Res Off of

College Station, TX

TEXAS NAT RESOURCE CON COMM

Austin, TX

TEXAS RES \& ENG INST

Grones, TX

$187400-0401-5$

$187400-0501-1$

$286550-0001.9$

$280600-0001-2$

TEXAS STATE OF

General Services Commission

Austin, TX

Governor's Off of Engy Res Con

Austin, TX

Governor's Ofice

Austín. TX

Health Dept of

Austin, TX

Resource Mgmt Border Issues

Austin, TX

TEXAS STATE TECHNICAL INST

Waco, TX

TEXAS TRANSPORTATION INST

College Station, TX

TEXAS UNTVERSITY OF

Austin, TX

Engineertug Division of

Austin, TX

El Paso. TX

San Antorio, TX

Nuclear Engineerine

Austin, TX

Pan Am

Austin, TX

Research/Sponsored Project Off

El Paso, TX

Sponsored Projects Otice of

Odessa, TX

$247340-1301-2$

$247340-1201-6$

$085180-0601-5$

$247340-1501-5$

$247340-1401-9$

109140-0003-9

$280610-0001-6$

$085210-0002-2$

$085210-3902-6$

$085210-3903-4$

$055210-3906-9$

085210-4902-1

085210-3702-3

$085210-4803-3$

$085210-4705-3$

TEXTRON DEFENSE SYSTEMS

Everett, MA

THEISEN PHILIP \& JAMES MCGRAY

Madison, WI

$78210-0001-9$

THEO BARRY \& ASSOC

Los Angeles, CA

THERESA B PRINCE SERVICES INC

Gaston, SC

THERMAL TRANSFER CORP

Monroeville, PA

276580-000]-9
THERMAL WINDOW WEAR

Rollingbay, WA

THERMO BLACK CLAWSON INC

Middletown, $\mathrm{OH}$

THERMO ENERGY CORP

Sunnyvale, CA

THERMORETEC CONSULTING CORP

Ithaca, NY

THERON CRALL

Oak Grove, MO
$280630-0000]-3$

$290350-0001-3$

$280640-0001-7$

$280650-0001-0$

$290340-0001-X$

$280660-000 \mathrm{~L}-4$

$291810-(000) 1-4$

283730-0001-1 
Awardec $\quad \begin{gathered}\text { Identiofication } \\ \text { code }\end{gathered}$

THIOKOL CORP

Brigham City, UT

THOMAS JEFFERSON UNIVERSITY

Philadeiphia, PA

THOMPSON C D

Rock Springs, WY

THOMPSON J

Devils Lake, ND

THOMPSON \$CHOOL DISTRICT

Regon 2-J

Loveland, $\mathrm{CO}$

THORNDAL-ARMSTRONG-DELK

Las Vegas, NV

THRESHER HANN JACKSON

Evansville, IN

THUROW LESTER C Cambridge, MA

TIC - THE INDUSTRLAL CO

Steamboat Springs, $C O$

$285370-0001-9$

120050-000l-9

$280670-0001-8$

280680-0001-1

TIGR SCIENCE EDUCN FDN INC Rockville, MD

TIMKEN CO

Steel Business Unit

Canton, $\mathrm{OH}$

TIYM PUBLISHING 00 INC McLean, VA

TOR ENGINEERING

Bonesteel, SD

Maricopa, AZ

Moorhead, $\mathrm{MN}$

New Underwood, SD

Washburn, ND

TORONTO UNIVERSITY OF

Ontario, Canada

Electrical Dept

Toronto, Canada

Mechanical Engineering

Toronto, Canads

TOSCO CORP

Phoenix, AZ

TOSCO FOUNDATION

Boulder, $\mathrm{CO}$

TOSHIBA AMERICA INFO SYS INC

Golden, CO

TOTAL ENERGY MANAGEMENT INC

Richland, WA

TOUCHE ROSS BAILEY SMART

San Francisco, CA

TOWNSEND \& BOTTOM INC

Salt Lake City, UT

TOWNSEND MARK A

Moscow, [D

TOWNSHIP OF MINE HILL

Mine Hill, N]

TRACER TECHNOLOGIES INC

Cambridge, MA

TRACOR MARINE INC

Port Everglades, FL
085440-0101-7

$287230-0001-5$

268790-0101.7

$280690-0001-5$

$281130-0001-1$

240310-0002-2

291380-0101-2

249590-0002-3

249590-0005-8

249590-0003-!

249590-0004-X

249590-0006-6

085910-0003-X

$085910-0101-X$

085910-0201-6

085920-0003-3

$280740-0001-\mathrm{X}$

224670-0002-B

$286900-0001-5$

280750-0001-3

$280780-0001-4$

$273050-0(0) 1-9$

$284190-0(0) 1-9$

086090-0002-8

280790-0001-8
264510-0001-9
Awardee

Identification

code

TRACTION PROPULSION

Austin, TX

280800-0001-8

TRAINING DELIVERY SERVICES INC

Eremont, CA

$289340-0001-5$

TRAINING GROUP

Fort Waslington, PA

$08612000008-4$

Montgontery, $\mathrm{OH}$

Oak Ridge, TN

Winchester, $\mathrm{OH}$

$086120-0005-\mathrm{X}$

$086120-0007-6$

$086120-0006-8$

TRANE CO

Asset Management

S1 Paul, MN

$29180020101-7$

TRANS \& ECONOMIC RES ASSOC INC Arlington, VA

$086150-0002-6$

TRANS ENERGY SYSTEMS INC Bellevise, WA

2808 10-0001-1

TRANSMISSION LINE TREE SERVICE

Grants Pass, OR

$290630-0001-4$

TRANSMISSION TECH

Fairfieid, NJ

$280820-0001-5$

TRANSNUKLEAR GMBH

Hanau, Germany

TRANSPIX FERRITER JNT VENTURE Jersey City, NJ

TRANSPORT INTER CORP

Carmel, CA

$280830-0001-9$

TRANSPORTATION DEPARTMENT OF

Mclean, VA

Puebio, CO

Const Guard

Alameda, CA

Systems Center

Cambridge, MA

$290100-0001-X$

$280840-0001-2$

TRANSTECTOR SYSTEMS INC Haydeo Lake, ID

$086260-0022-7$

$0 \$ 6260-0006-5$

$0 \$ 6260-0123-1$

$085260-1302-7$

$279510-0001-9$

TRAVEL MANAGEMENT CENTER

\$t Petersburg, FL

TRAVIS COUNTY EXT ENG COMM Austin, TX

$25 \$ 340-000 \mathrm{~J}-0$

TRAVIS ELEMENTARY SCHOOE Odessa, TX

TREASURY DEPARTMENT OF

Stattle, WA

Engraving \& Priating Bureau of

Washington, DC

$280850-6001-6$

Federal Law Enforcement Trng

Albuquerque, NM

The Mint Bureau of

Washington, DC

$280860-0001 \cdot x$

086290-0009-0

$086290-0802-4$

$086290-1304-4$

TREIMER DENNIS \& KENNETH MULLE Tipton, LA

TRENTON CITY OF

Admin Dept

Trenton, NJ

TRENTON R-IX SCHOOL DISTRICT

Trenton, MO

$264280-0001-6$

TRESCO INDUSTRIES LTD

Calgary, Cariada

$086350-0101-5$

$282930-0001-5$

$288290-0001-9$ 
Identification
Awarde

TRI ARK INDUSTRLES INC

Sonthampton, PA

291720-0001-5

TRI STAR COMPUTER Phoenix, AZ

TRI STATE ALT FUELS COALITION

OKI Regional Council of Govt

Cincinnati, $\mathrm{OH}$

$286140-0101-0$

TRI-COUNTY ECON DEV ALLIANCE

Barriwell, SC

TRI-STATE CLEAN AIR COMMITTEE

Cincitnati, $\mathrm{OH}$

$263970-0001-X$

TRI-STATE GENERAL INC Suit]and, MD

$280870-0001-3$

TRI-VALLEY SCHOOL LUBRARY

Healy, AK

243750-0001-5

TRIBAL EXECUTTVE BOARD

Assinthoine \& Stoux

Poplat, MT

TRIDENT ENGINEERING ASSOC INC Annapolis, MD

$280890-0001-0$

TRIDENT MANUFACTURING INC Plano, TX

$285150-0001-6$

TRILLIAM THERMO TECHNOLOGIES Pacific City, OR

TRIMBLEHOUSE CORP Noreross, GA

$292240-0001-0$

TRINITY COLLEGE

Washington, DC

TRINITY UNIVERSITY

San Anlonio, TX

$284080-0001-2$

TRISTAR COMPUTER Phoenix, AZ

TRITON COLLEGE DIST \#5044

River Grove, 片

$086580-0002-8$

086630-0001-4

290590-0001-3

TRITON MANAGEMENT \& GOVERNMENT Washington, DC

TRITON SERVICES INC

Easion, PA

TRODYNE CORP Teterboro, NJ

TRONLX ALEX Fresno, CA

TROSTLE VERNE W Kaanakakai, HI

TROUTMAN KEVIN Apple Creek, OH

TROW RICK PRODUCTIONS Willow Grove, PA

TROY SYSTEMS INC Fairfax, VA

TRUCKING RESEARCH INSTITUTE Alexandria, VA

TRUE POWER INC Las Vegas, NV

TRUST TERRITORY PACIFIC Saipan, $A Q$

TRUTECH LLC Idaho Falls, ID

$280940-0001-5$

$271450-(000]-6$

$280950-0001-9$

$224050-0002-4$

279550-0001-3

283480-000 I-1

$280960-0001-2$

$285000-000]-5$
Awardet

Identification sode

TS INFOSYSTEMS INC

Oxen Hil], MD

$083870-0002-7$

TUCSON CITY OF

Tueson, AZ

TUCSON ELECTRIC POWER

Tucson, AZ

TUCSON UNIFIED SCHOOL DIST \#1

Tucson, AZ

TUESDAY EVENING OPTIMIST CLUE

Casper. WY

TUFF ERNEST

Tuff Museum

Rushford, MN

$056880-0001-8$

$289610-000]-2$

$00-0001-6$

280990-0001-3

TUFTS UNIVERSITY

Grants \& Contracts Admin

Medford, MA

$266490-0101-5$

TULANE UNIVERSITY MEDICAL CTR

Now Orleans, LA

$291750-0001-6$

TULARE JOINT UHST

Tulare, CA

TULSA ENERGY RESOURCES

Tulsa, OK

[77620.0001-]

TULSA UNIYERSITY OF

Research Department

$281000-0001-8$
Tulsa, OK

TUNED SPHERE INTERNATIONAL

Nashua, NH

TURLOCK IRRIGATION DIST

Ttrlock, CA

TURNER HARPER \& ASSOC INC

Gaichersburg. MD

TURNER MASON SOLOMON

Dallas, $T X$

TUSCALOOSA CTY IND DEV AUTH

Tuscaloosa, AL

TUSKEGEE INSTITUTE

Tuskegee; AL

TUSKEGEE UNIVERSITY

Tuskegee, AL

TWIN FALLS PUBLIC LJBRARY

Twin Falls, iD

TWO EAGLE RIVER SCHOOL Dixon, $M T$

TWO-PHASE ENG \& RES INC

Santa Rosa, CA

TYLAN CORP

Tortance, CA

TYRRELL F \$

Port Richey, FL

$086950-0601-8$

28]1010-0001-]

087000.0001 .9

$253380-0002-4$

$087030-0001-X$

$281020-0001-5$

$087080-000 !-8$

$244260-0001-7$

$281040-0001-2$

$281050-0001-6$

291330-0001-8

$281060-000 \mathrm{~L}-\mathrm{X}$

$266610-0001-5$

$\mathbf{U}$

UD CTVILIAN RES DEVELOP FOUND

Moscow, USSR

$276860-0001-X$

UE \& C-CATALYTIC INC

Richland, WA

$281150-0001.9$

UINTEX

Salt Lake City, UT 
Awardee $\begin{gathered}\text { Identification } \\ \text { code }\end{gathered}$

ULLMAN TED

Ellensburg, WA

$280520-0001-7$

ULTRA SYSTEMS INC

Arlingion, VA

lrvine, $\mathrm{CA}$

McLean, VA

Newport Beach, CA

Chemistry Dept

Irvine, CA

ULTRAMAR INC

Houston, TX

UMATILLA NATIONAL FOREST

Pendleton, $O R$

UNDERGROUND INJ PRACT RES

Oklahoma City, OK

UNDERGROUND SYSTEMS

Arcadia, CA

Ammonk, NY

UNDERWRITERS LABORATORIES Melville, NY

UNLAX CORP

Sants Barbara, CA

UNIBUIIT INDUSTRIES INC

Vandalia, $\mathrm{OH}$

UNICOR

Er Reno, OK

Lexington-Fayette, $\mathrm{KY}$

UNICOR FED PRISON INDUSTRIES

Customer Service Center

Lexington, $\mathrm{KY}$

UNIFIED INDUSTRIES INC

Alexandria, VA

UNIFIED DNFORMATION INC

Kirkland, WA

UNION CARBDE CORP

Sisterville, WV

087430-0004-8

$087410-0001-3$

087410-0002-1

087410-0005-6

$087410-0101-X$

$204170-0003-0$

$281180-0001-X$

FND

$283100-0001-4$

$087480-0001-9$

$087480-0002-7$

$2811900-0001-3$

$292980-0001-4$

$281200-0001-3$

256310-0004-0

256310-0003-2

Líade Div

Indianapolis, IN

UNION OIL CO OF CALIFORNIA

Los Angeles, CA

Union Geothernul Div

Los Angeles, CA

$288920-0101-9$

$087530-0001-3$

$087710-0013.5$

$087710-0514-5$

$087860-0005-5$

Union Res Center

Brea, CA

UNION POWER CONSTRUCTION

Lake Havasu City, AZ

UNION SARAH COMM CORP

St Louis, MO

UNION SPRINGS CENTRAL SCH DIS

Union Springs, NY

UNIQUE MOBILITY INC

Englewow, $\mathrm{CO}$

Golden, $\mathrm{CO}$

UNIQUE SOLUTIONS

Reno, NV

UNISYS CORP

McLean, VA

087860-0205-8

087860-0106-X

178590-0003-2

$281210-0001-7$

$281220-0001-0$

$281230-0001-4$

$281230-0002-2$

$281240-0001-8$

195640-0002-5
$286020-0001-4$

Awardee

Identification

code

Federal Systems

McLean, VA

$195640-040210$

UNISYS Government Systems Inc

Hanover, MD

$195640-0303-2$

UNITAR

Tulse, $\mathrm{OK}$

$210880-0004-5$

UNITED BIOENERGY COMMER ASSOC

Columbia, MD

292140-0001-8

UNTTED CHURCH OF CHRIST CONGR

Hancock, NH

UNITED ENERGY TECH

St Perersburg, FL

$267370-0001-0$

UNITED INDIAN PLANNERS ASSOC

Washington, DC

$281250-0001-1$

UNITRD INDUSTRIES CORP

Bellevue, WA

$088010-000)]-4$

INITED NEGRO

$088020-0001-8$

Fairfax, VA

$136290-0002-9$

UNITED SOLAR SYSTEMS

Troy, MI

2868 t0-0001-2

UNITED SOLAR TECHNOLOGIES INC

Cabin John, MD

UNITED STATES COAL CONCLAVE

Washington, $\mathrm{DC}$

$233550-0002-7$

UNITED STEEL WORKERS

Pittsburgh, PA

UNITED TECHNOLOGIES CORP

West Palm Beack, FL

$281260-000]-5$

Combustion Sclences Dept

Hartford, IA

Power \& Industrial Syst Tech

Hartford, CT

$281270-0001-9$

Research Center

Eașt Hartford, CT

Hartford, IA

UNIV \& CMTY COLLEGE SYST OF NV

Las Vegas, NV

Chancellor's Office

Reto, NV

$098120-0005-3$

$088120-0108-4$

$088120-1307-4$

$088120-1101-2$

088120-1108-X

221630-0002-8

Desert Research Instilute

Las Vegas, NV

Reno, NV
UNIVERSAL ENGINEERING CO

Gaithersiburg, MD

UNIVERSAL OIL PRODUCTS

Des Plaines, IL

UNIVERSAL SYSTEMS INC

Albuquerque, NM

Chantilly, VA

UNIVERSE TECHNOLOGIES

Frederick, MD

UNIVERSITY CORP FOR ATMOS RFS

Boulder, $\mathrm{CO}$

UNIWORLD GROUP INC

New York, NY

UNO

Resenteh

New Orleans, LA
221630-0201-2

221630-0102-4

$221630-0101-6$

$28 \div 280-0001-2$

$281290-0001-6$

088180-0003-9

088180-0002-0

290830-0001-X

284840-0001-X

$088320-0002-4$

281100-0101-7 

Awardee
Identification
code

UP TIME DISASTER RECOVERY

Sacrasnento, CA

$281300-0001-6$

UPPER SAND MOUNTAN PARISH

Section, AL

$281310-0001 \cdot \mathrm{X}$

URBAN ENVIRONMENTAL

Washington, DC

$281320-000 \div-3$

URBAN INSTITUTE

Washington, DC

$281330-0001-7$

URS/TRANSENERGY SYSTEMS Portand, $O R$

$281340-0001-0$

US ADVANCED BATTERY CONSORTIUM Warten, MI

$215230-0001-5$

US COAST GUARD

Prosific Strike Team

Novato, CA

$281350-0202-5$

Research \& Dev Office of

Washington, DC

$281350-0101-0$

US COUNCIL FOR RENEWABLE ENGY

Washington, DC

$281140-0001-5$

US EXPORT CNCL FOR RENEW ENER

Washington, DC

$177670-0002-8$

US GEOLOGICAL SURVEY

Golden, $\mathrm{CO}$

Menio Park, CA

Reston, VA

$257240-00014-0$

$257240-00015-9$

257240.0003.2

Central Rexion

Denver, CO

Water Resources

Denver, $\mathrm{CO}$

$257240-0301-5$

$257240-0201-9$

US GREEN GUILDING COUNCIL

San Francisco, CA

US INDUSTRY COALITION INC

Washington, DC

US MILITARY ACADEMY

West Point Military Reserve, NY

Social Sciences Dept

West Point, NY

US NANOCORP INC

North Haven, CT

US NATL ALCOHOL FUELS COMM

Administration Chief of

Washington, DC

US NAYAL OBSERVATORY

Washington, DC

US OIL \& REFINING CO

El Segundo, CA

US OPTICS TECHNOLOGIES INC

Buena Park, CA

$285770-0001-x$

290190-0001-2

$281070-0002-1$

$281070-0101-\mathrm{x}$

$290070-0001-2$

\section{$M$}

$281080-0101-3$

292550-0001-2

088530-0004X

285490-0001-9

US PETROCHEM CORP

Contracts \& Grants

Santa Monica, CA

US POSTAL SERVICE

Golöen, $C O$

Washington, DC

US STEEL CORP

Monroville, PA

066370-0011-6

066370-0003-5

$087280-0001 \cdot 3$
Awardee

Identifieation

code

US TESTING CO

Richland Div

Richland, WA

US WATER RESOURCES COUNCIL

Washington, DC

$087290-0101-3$

Assessment

Washington, $D C$

USA CONSTRUCTION INC

Loveland, CO

USCO POWER EQUTPMENT CORP

Birmingham, AL

USS NOVAMONT ENC

New York. NY

UTAF BIOMEDICAL TEST LAB

Salt Lake City: UT

UTAH STATE OF

Community \& Ecouomic Dev Dept

Salt Lake City, UT

Conmunity Services Offlce

Salt Lake City, UT

Engineering Dept

Salt Lake City, UT

Transportation Dept

Salt Lake City, UT

Water Resources

Salt Lake Ciry, UT

UTAH STATE UNTVERSTTY

Contract \& Grant Office

Logan, UT

Cooperative Extension Service

Logan, UT

UTAH UNFVERSITY OF

Salt Lake City, UT

Connminution Center

Satt Lake Cíy, UT

UTILITY ELECTRIC SUPPLY CO

Dekalb, ll

Rapid City, SD

UTLEY ESQ MARGIE A

As]inglon, VA

UZIEL MOSHE

Northridge, CA

$2810900001-0$

$281090-0101-7$

$286330-0001-6$

287430-000(t)-0

$281370-0001-1$

$281380-0001-5$

$088630-0101-4$

088630-1501-5

$088630-1701-8$

088630-160]-1

$088630-180]-4$

$088640-0701-6$

$088640-0601 \cdot \mathrm{X}$

$088650-0001-5$

$088650-3601-\mathrm{X}$

$259470-0002-4$

$259470-0001-6$

$249150-0002-6$

281390-0001-9

V

VACHON NIX ASSOC

Norcross, GA

$281410-000 \mathrm{l}-2$

VACUDYNE INC

Chicago Heights, IL

VALLENY ELECTRIC ASSOC INC Mercury, NV

VALLEY CONTRACTING CO

Grand Fotks, ND

VALLEY FORD

Fargo, ND

VALLEY SCHOOL DISTRICT $\$ 262$ Hazelton, ID

VALLIVUE SCHOOL DISTRICT \#139

Caldwell, ID

$285810-000+10$

$291880-0001-X$

$281420-000]-6$

290460-0001-X

$283580 \cdot 0001-4$

$283550-40001-3$ 
Awardee

VALMONT INDUSTRIES INC

Valky, NE

VALUE ENGINEERING CO

Alexandria, VA

VAN BUREN TOWNSHIP

Belleville, Ml

VANGUARD ASSOC INC

Washinglon, DC

VARCOM

Englewood, $\mathrm{CO}$

VARIAN ASSOC INC

Lexinglon, MA

Palo Alto, CA

Solid State Laboratory

Palo Alto, CA

VARIGAS RESEARCH INC

Timonium, MD

VEDA INC

Camarillo, CA

VEGA CORP OF TENNESSEE

Chattanooga, TN

VELCON FILTERS

Colorado Szpings, co

VELSO J

Tampa FL

VENDE VALLEY SOLAR ENERGY

Sedona, AZ

VENTURA COLLEGE

Mathematies

Ventura, $C A$

VENTURE CATALYST INC

Tucson, AZ

VERDEL THOMAS H

OkJahoma City, OK

VERMONT ELECTRIC COOP INC Johnşon, VT

VERMONT ENERGY INVESTMENT CORP

Burlington, VT

VERMONT MARBLE CO

Proctor, VT

VERMONT RESEARCH CORP

North Springfield, VT

VERMONT UNIVERSITY OF

Chemistry

Burlington, VT

VERMONT YANKEE NUCLEAR POWER

Rutland, VT

VETERANS ADMIN

Gainesvifile, FL

Sopply Service

San Diego, CA

VETERANS AFFAIRS DEPT OF

Golden, $\mathrm{CO}$

Wastington, DC

Lebanon Medical Center

Lebarion, PA

VETTER RESEARCH

Cosia Mesa, CA
Identification

code

257680-0001-1

281430-0001-X

$281440-0001-3$

$089030-0002-1$

$289360-0001-2$

089060-0003-0

$089060-0001-4$

$089060-0105-0$

089070-0001-8

$281460-0001-0$

$089130-0002-4$

$259190-0002-3$

$270290-0001-3$

$281480-0001-8$

$287750-0101-2$

$292920-000 \mathrm{~L}-2$

267590-0001-3

089180-0001-4

289600-0001-9

281490-0001-1

$281500-0001-1$

089230-070l-3

089250-0002-4

089340-0006-6

$089340-0407-x$

2.15210-0003-4

215210-0005-0

$215210-0204-5$

281510-0001-5
Awardet

Identification

code

VGS INC

McLean, VA

VIC'S MOAL-ARC INC

Lafayette, $\mathrm{CO}$

VICON INDUSTRITS INC

Norctoss, GA

VICTOR INSULATORS

Orinda, CA

VIETH DR DONALD L

Maineville, $\mathrm{OH}$

VILLANOVA UNTVERSTTY

Chemitesl Engineering Dept

Viltanowa, PA

VINCENNES UNIVERSITY

Vincennes, $\mathbf{N}$

VINCENT JOHN R

San Juan Pueblo, NM

VINDICATOR TECHNOLOGIES

Austin, TX

VIRGIN ISLANDS COMMONWEALTH OF

Energy Office

Christiansted, VI

VLRGINLA BAPTLST HOSPITAL

Princess Anne, M5

VIRGINIA COMMONWEALTH OF

Richmond, VA

Benefts Programs Div

Richmond, VA

Corporate Commlsslon

Richmond, VA

Corrections Dept of

Lorton, VA

Edmeation Dept

Richmond, VA

Emergency Services Dept

Richmend, VA

Eravironmental Quality Dept of

Richmond, VA

Housing Comna Devel Dept of

Richmond, VA

Mines Mimerals \& Energy Dept

Richmond, VA

Police

Richmond, VA

Solid \& Hazardous Waste

Richmond, VA

265050-0001-1

$281520-0001-9$

278330-0001-9

$292470-0001-7$

$278240-0001-X$

089500-0301-5

$281540-0001-6$

$270900-0001-X$

$292110-0001-7$

$08957)-0106-9$

$281560-0001-3$

$089680-0001-8$

$089680-0401-3$

$089680-0101-4$

089680-1002-1

089680-0901-5

089680-031)1-7

$243350-1201-2$

243350-0201-7

$089660-0801-9$

$089680-1101-x$

$089680-0601-6$

Solid Waste Commission

Richmond, VA

VIRGINIA COMMONWEALTH UNIV

Vtrginia Merictal College

Richmond, VA

VIRGINIA CORP OF RICHMOND

Richmond, VA

VIRGINIA EASTERN SHORE EEHC

Economic Empowerment Housing

Nassawadox. VA

$286560-0101-9$

VIRGINIA INST OF MARINE SCI

Gloncester Point, VA

$286700-0001-6$ 

Awardee
Identification
code

VIRGINIA MEDICAL COLLEGE

Richmond, VA

281620-000L-I

VIRGINIA POWER

Richmond, VA

$291230 \cdot 0001-5$

VIRGINIA RESEARCH INSTIYUTE Arlington, VA

$281630-0001-5$

VIRGINIA SETTLERS ENERGY CTR

Biacksburg. VA

$281580-0001-0$

VIRGINLA TRANSFORMER CORP Rọnoke, VA

$089700-000]-1$

VISTA CONTRACTING INC

Washington, DC

VISTA TECHNOLOGIES INC Washington, DC

VITRO LABS DIV

Silver Spring: MD

VLOC INC

Tarpon Springs, FL.

VOGEL DITHMAR STOTLAND Chicago, IL

VPT INC

Blacksburg, VA

VREDENBURG

Reston, VA

VSN \& ASSOC

Ricbland, WA

293080-0001-1

$224440-0002-1$

$281590-0001-4$

$252180-0001-9$

089840-000I-9

$2899900001-x$

289900-0001-7

$286820-000]-6$

$\mathbf{W}$

W B HEFLIN PUMPING SERVICE Blythe, CA

287860-0001-2

W BRANDT GOLDSWORTHY \& ASSOC Torrance, CA

W S FLEMTNG \& ASSOC INC Hyattsville, MD

W W WALLWORK INC Bismarck, ND

WA ASSOC OF BLDG OFFICLALS Seattle, WA

WACKENHUT SERVICES INC Wastington, DC

WADE TROY Las Vegas. NV

292260-0001-8

$281660-0001-6$

$288740-10001-4$

$281700-0001-7$

$090020-00006-5$

WAGNER KARL H

Fewortamue Hlsur 5, Georgia

Munich, Germany

WAGNER NELDON

Kodjak, AK

WALKER DEMPSEY L Independence, KS

WALKER DORSEY H Section, AL

WALKER JR JOHNINIE B Shreveport, LA

WALKERS ELECTRONICS Altadena, CA WALLACE SCHOOL DISTRICT \#393

Wallace, ID

$266660-0001-3$

$090090-0002-8$

$090090-0001 \cdot \mathrm{X}$

$275120-090 !-4$

$266150-000]-X$

264750-0001-9

268160-000]-3

$289740-0001-6$

$264900-0001-6$
Awardee

Identification

code

WALTERBORO CITY OF

Walterboro, \$C

$281740-0001-1$

WAND LABS INC

Albuquerque, NM

281750-0001-5

WANDEL \& GOLTERMANN INC

Parkwood, NC

$166950-0009-3$

WANG LABORATORIES INC

Clinton, MD

090430-0016-7

WAPORA INC

Washington, DC

WARBONNET ELECTRIC INC

Blackfoot, it

$090450-(000)-6$

WARD WILLIAM H

Shawnec Mission, KS

$096760-0002-7$

WARNER JACK R SI John, VI

$281780-0001-6$

090480-0001-7

WARREN HILLS REGIONAL SCHOOLS

Washington, N]

$0905300002-\mathrm{X}$

WARREN SCHOOL DEFT

Warten, RI

$148460-0001 \cdot X$

WARREN WEIL ASSOC INC

Washitiglon, DC

WARSHOW JOHN

Plainficłd, VT

$281790-000]-X$

WASHINGTON COUNTY HOSPITAI

Hagerstown, MD

$270980-0001-9$

WASHINGTON CO

St George, UT

WASHINGTON ORG OF SMALL TRIBES

Sumner, WA

$090740-0001-0$

WASHINGTON STATE OF

Community Trade \& Economite Dev

Olympia, WA

Maragement \& Budget Office of

Washington, DC

WASHINGTON UNIVERSITY St Louis, MO

WASHINGTON UNIVERSITY OF

Forest Resoutres College of

Seattle, WA

DIST

$137220-0002-5$

$281800-0001-X$

Jnterdiscipline Arts \& Sci

Bothell, WA

$090850-1901-X$

090850-2108-1

$090890-0004-6$

WASTE MANAGEMENT

Great Falls, MT

Sioux City, IA

WASTE MANAGEMENT OF N DAKOTA

Bismarck, ND

2900001-3

WASTE MANAGEMENT OF PHOENIX Phoenix, AZ

$286680-0001-2$

WASTETRON

Poca, WV

$290320-0001-2$

WASTREN INC

Aiken, $S C$

WATER BUDGET CENTER

Pordand, OR

$247610-0003-1$

WATER RESOURCES RESEARCH INST

Laramie, WY

$281830-0001-0$ 


$$
\text { Awardee Identification }
$$

WATERHOUSE PROCESS \& CO Washington, DC

WATERJET TECHNOLOGY INC Kent, WA

WATERL OO UNIVERSITY OF Waterloo, Canada

WATERMAN EDWARD L

Fort Collins, CO

WATLINGTON LUIS $\mathrm{E}$

Hato Rey, RQ

WATSONVILLE CITY OF

Commurnity Developmest Dept

Watsonville, CA

WATT ENGINEERING LTD

Cedarodge, $C O$

WATTS DEY

Green Bay, WI

WAUKESHA ELECTRIC SYSTEM

Waukestra, WI

WAUSAU HOMES INC

Wausau, W1

WAVERLY CITY OF

Waverly, $\mathrm{OH}$

WAYLAND BAPTIST UNIVERSITY

Plainview, TX.

WCKENHEISE ROBERT J

Emmisburg, MD

WEBB DR L DALE

College Station, TX

WEBB LAW FIRM

Pittsburgh, PA

WEHRLIS ROBERT

Potomac, MD

WEIL ROBERT $\mathrm{C}$ New London, CT

WEITZENHOFF MICHAEL H Honchilu, HI

WELCH MURELL L Mount Pleasant, MI

WELCOME TRUST

London, United Kingdon

WELD COLNTY AMBULANCE SERVICE Greeley, $C O$

WELD COUNTY SCHOOL DISTRICT

Region 9

Auㅣㄴ $\mathrm{CO}$

WELD COUNTY SHERIFF OFFICE

Gretey, $\mathrm{CO}$

WELLBORN JOHN

Atlanta, $G A$

WELLS OTHO S

Durham, NH

WELLS ST JOHN \& ROBERTS Spokane, WA

WENDELL SCHOOL DISTRICT \#232

Wendell, ID

WESCO DISTRIBUTION INC

Bismank, ND
$281840-0003]-4$

$283270-0001-2$

$281850-0001-8$

$121370-0001-6$

$272590-0001-5$

$290550-0101-5$

$281860-0001-1$

$281870-0001-5$

289440-0001-8

269830-0001-3

$281880-0001-9$

$281890-0001-2$

$277480-0001-8$

$265200-0001-9$

283000-(K)01-1

$281910-0001-6$

$281930-0001-3$

$273760-0001-1$

$281950-0001-0$

$287180-0001-0$ $283330-0001-1)$

$091570-0101-4$

278290-0001-8

$281960-0001-4$

$281970-0001-8$

$091650-0001-3$

$279710-0001-4$

$289080-0001-1$
Awardee

Identification code

WESSLING CONSULTING

Albuquerque, NM

WEST ANGELES CMTY DEV CORP

Los Angeles, CA

WEST CENTRAL COMMUNITY CENTER

Spokane, WA

282010-0001-3

WEST HARLEM ENVIRONMENTAL ACT

New York, NY

$290760-0001-8$

WEST HIGH MEDIA CENTER

Anchotage, AK

282020-0001-7

WEST M G

San Francișeo, CA

285830-0001-8

WEST MICHIGAN ENVIRON ACTION

Earth Day 80

Grand Rapids, MI

$282030-0101-7$

WEST PENN POWER CO

Pittsbugh, PA

WEST PIEDMONT PLANNING

Martinsville, VA

WEST ROBERT W

Chester, MD

WEST TEXAS COUNCIL OF GOVMTS

Economic Development Dept

El Paso, TX

WEST VIRGINIA STATE OF

Charleston, WV

$092040-0003-1$

Euvironmental Protection Dept

Morgantown, WV

Fuel \& Enery Ofifice

Charleston, WV

WEST VIRGINIA UNIVERSTTY

Research Corp

Morgantown, WV

WESTAT INC

Rockville, MD

WESTBERG II JOHN $\mathrm{K}$

Smithfield, ME

WESTEC SERVICES INC

San Diego, CA

$282040-0001-4$

$277570-0(0)]-7$

Imper Val Op Ofc

San Diego, CA

WESTECH INTERNATIONAL INC

Albuquerque, NM

$262910-0101-2$

092200-0001-6

$092200-0802-5$

$092200-0305-5$

$092210-2401-6$

$199720-000]-X$

$282050-0001-8$

$092320-0001-6$

Q92320-0101-2

WESTERN AGGREGATE INC Marysvilje, CA.

$265010-0001-7$

$279500-0001-5$

WESTERN BUILDING SERVICES

Golden, $\mathrm{CO}$

WESTERN ENERGY INC

Palo Alto, CA

289920-0001-4

WESTERN FOUNDRY \& MACHINE WORK Topeka, KS

282060-0001-]

WESTERN GOVERNORS' ASSOC

Denver, CO

$092450-0001-\mathrm{X}$

WESTERN INTERSTATE COMMISSION

Boulder, $\mathrm{CO}$

$282070-000]-5$

WESTERN INTERSTATE ENERGY BD

Denver, CO

092480-0001-0 

Awardee
Identification
code

WESTERN INTERSTATE NUCLE

Lakewood, 00

282080-0001-9

WESTERN INTST COMM HI ED

Boulder, $C O$

$282090-0001-2$

WESTERN OFFICE SYSTEMS INC

Golden, CO

$122550.0002-4$

WESTERN OIL SHALE CORP

Las Vegas. NV

$282100-0001-2$

WESTERN ONTARIO UNIVERSTTY OF

Physics Dept

London 72, Canada

$282110-0101-2$

WESTERN REGION SALES

Suntryvale, CA

$265260-0001-0$

WESTERN SOLAR UTHL NETWORK

Porltand, OR

WESTERN TECHNICAL ASSOC

Los Angeles, CA

$282120-000]-X$

WESTERN UNITED

Denyer, $C O$

WESTERN WA SOLAR ENERGY ASSN

Seatlle, WA

[52210-000]-6

WESTERN WASH STATE COLLEGE

Bellingham, WA

WESTERN WYOMNG COLLEGE

Water Lab

Rock Springs, WY

$282170-0101-4$

WESTINGHOUSE ELECTRIC CORP

Des Moitres, LA

WESTINGHOUSE $R$ \& $D$ CENTER

Pittsburgh, PA

092680-0052-0

WESTINGHOUS 282180-0001-1 Jamestown, ND

WESTPORT TECHNOLOGY CENTER Houston, TX

WESTWOOD COMPUTER CORP

Intormation Management Div

Springfield, NI

WEYAUWEGA SCHOOL DISTRICT

Fremont, WI

$196160-0163-5$

WHARTON ECON FORECASTING INC

Philade]phia, PA

Washington, DC

WHEATLAND HIGH SCHOOL

Whealland, WY

SVCS

242890-0002-9

284970-0001-3

WHEELED COACH INDUSTRIES INC

Winter Park, FL

WHEELER HENRY

Wilmington, VT

WHITAKER BROTHERS

San Diego, CA

WHITE HOUSE

Washington, DC

WHITE HOUSE CONF ON AGE

Washinglon, $\mathrm{DC}$

WHITEHEAD N L

Srrawbery Plains, TN

WHITEPINE JOINT \$CH DIST \#286

Troy, ID

6

$092960-0001-7$

$092960-0002-5$

$282200-0001-5$

245990-0001-9

$269180-0001-2$

$267760-0001-8$

$282210-0001-9$

$282220-0001-2$

$274560-0001-8$

279730-0001-1
Awardee

Identification

code

WHITNEY MECHANICAL CO INC

Kremmling $\mathrm{CO}$

288860-0001-4

WHITTED III HUGH

Enst Bend, NC

$282230 \cdot 0001 \cdot 6$

WHITTLESEY PROF NORMAN $K$

Palouse, WA

$282240-0001-\mathrm{X}$

WHKES TOM

Yakima, WA

280720-0001-2

WHOLESALE INDEPENDENT ELECTRIC

Pensacola, FL

WILKES COLLEGE

Wilkes-Bame, PA

$288450-0001-X$

WILKINS SYSTEMS INC

Washington, DC

WILL CITY REGIONAL SCHOOL

Joliet, IL

WILL COUNTY REG SCHOOLS

Joliet, JL

WILL-BURT CO

Orrville, $\mathrm{OH}$

$28226000001-7$

$093430-0002 \cdot 2$

$282270.0001-0$

$282280-000]-4$

$291660-10001.7$

WILLIAM \& MARY COLLEGE OF

Physics Dept

Charlotterville, VA

WILLIAMS BROTHERS ENG CO

Godsey-Earlougher Div

Tulsa, OK

WHLLAMS COLLEGE

Williamstown, MA

WILLIAMS J B

Northridge, CA

WLLIAMS MARK A

Moscow, ID

WLLLAMS O $G$ \& SKAGGS R $L$

Las Vegas, $\mathrm{NV}$

WILLIAMS RESEARCH CORP

Walled Lake, MI

WILLIAMS THOMAS B

Mtiddletown, PA

WILSEY \& HAM

Foster City, CA

WLLSON CITY OF

Wilson, NC

WILSON FIRE PROTECTION

Tulsa, OK

WLLSON PAUL B

Florissant, MO

WIND POWER ASSOC

Amherst, MA

WINDCATCHER COMPANY

Sutton, NE

WINDFARM MUSEUM

Vineyard Haven, MA

WINDHAM JAMES R

Gainesvifle, FL

693480-0104-3

093570-0301-0

$282370-0001 \cdot 3$

$282380-0001-?$

282390-0001-0

$282400-0001-0$

$282410-0000]-4$

267550-0001-9

$2824200-0001-8$

282430-0001-]

$282440-0001-5$

$282450 \cdot 0001-9$

$282460.0001-2$

$282470-0001-6$

282480-0001-X

270500-0001-9

WINKER FRED

Prior Lake, MN

$282490-0001-3$

WTNSTON \& STRAWN

Washington, DC 
Awardce Identification

WINSTON CORP

Englewood, CO

WINTERGREEN COOP SOLAR GH INC Orange, MA

WINYARD FREDERICK

Oakland, CA

WIRTH ASSOC

Billings, MT

WISCONSIN CTR FOR PUB POLICY

Madison, WI

WISCONSIN PUBLIC SERVICE CORP

Green Bay, WI

WISCONGIN STATE OF

Admin Dept of

Madison, WI

Admin Dept of / Energy Div

Madison, WI

Energy \& Intergovt Rel Div of

Madison, WI

Eneroy Divisinn

Madison, WI

WISCONSIN STOUT UNIVERSITY OF

Graphic Communication Dept

Menomonie, WI

$282530-0101-0$

WISCONSIN UNIVERSITY OF

Electrical \& Computer Eng Dept

Madison, WT

Entomolos

Madison, WI

WITT CHARLES $K$

Las Cruces, NM

WIZARD RES \& DEV GROUP INC

Washistgton, $\mathbf{D C}$

$094110,0101-7$

$0941] 0-1601-4$

$094110-1701-0$

$094110.1501-8$

WKM WELLHEAD SYSTEMS

Shreveport, LA

WLVEC TECHNOLOGY COMPANY

Adel, $\mathrm{L}$

WOLF RUN IRRIGATION COOP INC

Dufur, OR

WOLVERINE ELECTRIC

Big Rapids, MI

WOOD DOUG

Fox [s]and, WA

WOOD ENERGY INSTITUTE

Waitsfreld, VT

WOODHOUSE ROBERT $P$

Slivell, LA.

094130-080I-9

$094130-5101-1$

$282540-000 \mathrm{I}-8$

$282550-0001-1$

$281650-0001-2$

$282560-0001-5$

282580-0001-2

$282590-0001-6$

$282600-0001-6$

WOODS HOEE OCEANOGRAPHIC INST

Chemistry Dept

Woods Hole, MA

WOODS HOLE RESEARCH CENTER

Boston, MA

$282610-0001 \cdot X$

WOODWARD MARY VICTORIA

Port Deposit, MD

WOODWARD-CLYDE CONSULTANTS

Scoville, ID

WORF DOUGLAS L

Cary, NC
Awardee

Identification code

WORKMED

Tulșa, oK

282620-0001-3

WORLD PHYSICS TECHNOLOGIES INC

Blackshurg, VA

WORTHAN ANDREW

Santa Monica, CA

$259030-0001+0$

$282630-0001-7$

WRANGELL PUBLIC SCHOOLS

Wrangell, AK

WRIGHT CITY R-II SCHOOL DIST

Wright City, MO

WRIGHT LINE INC

Reston, VA

WRIGHT MALTA CORP

Ballston Spa, NY

Maita Test Station

Baliston Spa, NY

WROBLE JERRY

Anchorage, AK

WSF ASSOC

Burke, VA

$282640-0001-0$

282940-0001-9

$094690-01006-4$

282650-10001-4

$282650-0101-0$

$282660 \cdot 0001-8$

WT SERVICES INC

Amarillo, TX

WUESTERBERG DEAN

Donahue, lA

WV HIGH TECHNOLOGY CONSORYHUM

Fairmont, WV

WYATT CO

Chicago, IL

WYCO MECHANICAL SERVICES

Loveland, CO

282670-0001-1

$285160-0001-X$

WYLE LABORATORIES ENC

El Segundo, CA

WYOMING ANALYTICAL LABORATORY

Laramie, WY

WYOMING STATE OF

Family Service Dept of

Cheyenne. WY

WYOMING UNIVERSITY OF

Energy Restarch \& Tech Corp

Laramie, WY

$258870-0002-3$

$094750-0002 \times$

$287890-0001-3$

$0948(X)-0002-4$

$282680-0001-5$

$094850-0701-9$

$094860-1801-4$

$\mathbf{X}$

XAVIER UNIVERSITY OF LOUISLANA

New Orleans, LA

207370-0601-5

Phystes \& Engineering Depts

New OTleans, LA

207370-0101-1

XENERGY

Lexington, MA

XEOTRON CORP

Houston, TX

XEROX CORP

Albuquerqque, NM

St Petersburg. FL

$282690-0001.9$

289320-000!-8

094900-0009-4

094900-0033-7

Electro-Optical Systems

Pasadena. CA

$094900-0938.5$

Research Center

Palo Alto, CA

094900-0837-0 
Awardee

XEROX ENGINEERING SYS

Herndon, VA

XL ASSOC INC

Rockrille, MD

XL MICROWAVE

Bloomingdale, IL

XTALONJX PRODUCTS

Columbus, $\mathrm{OH}$

XYZYX INFORMATION CORP

Wellesley, MA

$$
\mathbf{Y}
$$

Y Y LABS INC

Fremont $C A$

YAKIMA VALLEY CONF OF GOVT

Yakjma, WA

VAKUTAT CITY \$CHOOLS

Yakutat, A.K

YALE SCHOOL OF MEDICINE

Human Genetics

New Haven, CT

YALE UNIVEKSITY

New Haven, $\mathrm{CT}$

Applied Mecbsnics Dept

New Haven, CT

Political Sctience New Haven, CI

YANCY MINERALS INC Boston, MA

YAP INST OF NATURAL SCIENCE Yap, AK

YARDNEY TECH PRODUCTS

Pawcatuck, CF

YARNELL RICHARD C Carson City, NV

YAYAPAI COUNCIL ON AGING Prescolt, AZ

YELLOW CAB CO OF DC

Washington, DC

$289350-0001-9$

$282710-0001-2$

$282720-0001-6$

$282730-0101-6$

$094960-0001-0$

$094960-1901-3$

094960-2001-I

094970-0004-9

282740-0001-3

289190-0001 8

$277280-0001-2$

$282750-0001-7$

$288890-000 \div-5$

YELLOW SPRINGS OPTICAL SENSOR

Yellow Springs, OH
Awandee

Identification

code

YESHIVA UNIVERSITYY

Medical School

Botarical, NY

YING MANUFACTURING CORP Gardena, CA

$095190-0103-4$

$282760-0001-0$

YOLO EMPLOYMIENT SERVICES

Woodland, CA

YORK INTERNATIONAL CORP

Las Vegas, NV

293010-0001-6

179900-0003-2

YORK RESEARCH CORP

Engineering Services

Stamford, CT

YOUNG CREATIVE SOLUTIONS

Eustis, FL

YOUNG FRED L

Midwest City, OK

YOUNG LAFAYETTE

Haiku, $\mathrm{HI}$

$\boldsymbol{Z}$

$Z$ INC

Silver Spring, MD

ZBB TECHNOLOGY INC

Wauwatosa, WT

ZEBRA EQURPMENT INC

La Mirada, CA

ZENITH DATA SYSTEMS

Sacramento, CA

ZETA PHI BETA SORORITY

Washinglon, DC'

ZIEGLER LOXD T

Ozark, AR

ZIFF-DAVIS EDUCATION

Henderson, $\mathrm{NV}$

Redmond, WA

ZUNEEL TREE SVC

Sleamboat Springs, $\mathrm{CO}$
$260300-0001-2$

$282770-0101-0$

286920-0001-9

$267410-0001-1$

271780-0001-5

$285710-0001-8$

$285930-0001-0$

I36590-0003-5

$292160-0001-5$

$272410-0001-3$

287350-10002-3

$287350-0001-5$

287420-0f0l-7 